\title{
Lie bialgebras, Poisson Lie groups and dressing transformations
}

\author{
Yvette Kosmann-Schwarzbach \\ Centre de Mathématiques, UMR 7640 du CNRS, \\ Ecole Polytechnique, \\ F-91128 Palaiseau, France \\ e.mailyks@math.polytechnique.fr
}

Integrability of Nonlinear Systems, Second edition, Lecture Notes in Physics 638, Springer-Verlag, 2004, pp. 107-173.

\begin{abstract}
In this course, we present an elementary introduction, including the proofs of the main theorems, to the theory of Lie bialgebras and Poisson Lie groups and its applications to the theory of integrable systems. We discuss $r$-matrices, the classical and modified Yang-Baxter equations, and the tensor notation. We study the dual and double of Poisson Lie groups, and the infinitesimal and global dressing transformations.
\end{abstract}

\section{INTRODUCTION.}

SeCtion 1. Lie bialgebras

1.1 An example: $\mathfrak{s l}(2, \mathbb{C})$

1.2 Lie-algebra cohomology

1.3 Definition of Lie bialgebras

1.4 The coadjoint representation

1.5 The dual of a Lie bialgebra

1.6 The double of a Lie bialgebra, Manin triples

1.7 Examples

SECTION 2. Classical Yang-Baxter equation and r-matrices

2.1 When does $\delta r$ define a Lie-bialgebra structure on $\mathfrak{g}$ ?

2.2 The classical Yang-Baxter equation

2.3 Tensor notation

$2.4 R$-matrices and double Lie algebras 
2.5 The double of a Lie bialgebra is a factorizable Lie bialgebra

SECTION 3. Poisson manifolds. The dual of a Lie algebra. Lax equations 3.1 Poisson manifolds

3.2 The dual of a Lie bialgebra

3.3 The first Russian formula $\{L \stackrel{\otimes}{,} L\}=[L \otimes 1+1 \otimes L, r]$

3.4 The traces of powers of Lax matrices are in involution

3.5 Symplectic leaves and coadjoint orbits

3.6 Double Lie algebras and Lax equations

3.7 Solution by factorization

SECTION 4. Poisson Lie groups

4.1 Multiplicative tensor fields on Lie groups

4.2 Poisson Lie groups and Lie bialgebras

4.3 The second Russian formula (quadratic brackets) $\{L \stackrel{\otimes}{,} L\}=[L \otimes L, r]$

4.4 Examples

4.5 The dual of a Poisson Lie group

4.6 The double of a Poisson Lie group

4.7 Poisson actions

4.8 Momentum mapping

4.9 Dressing transformations

Appendix 1. The 'big bracket' and its applications

Appendix 2. The Poisson calculus and its applications

\section{SELECTED BIBLIOGRAPHY}

\section{Introduction}

What we shall study in these lectures are classical objects, not in the sense that they date back to the nineteenth century, but in the sense that they admit a quantum counterpart. In fact, the theory of Lie bialgebras and Poisson Lie groups, due for the most part to V. G. Drinfeld and M. A. Semenov-TianShansky, dates back to the early 80's, while the concept of a classical $r$-matrix was introduced a few years earlier by E. K. Sklyanin. It is somewhat surprising that these structures first appeared as the classical limit (the expression "semi-classical limit" is sometimes used instead) of the mathematical structures underlying the quantum inverse scattering method (QISM) developed by L. Faddeev and his school in St. Petersburg (then Leningrad). The commutativity property of the row-to-row transfer matrices for solvable lattice models was found to be a consequence of the existence of the so-called quantum $R$-matrix, which figures in the now famous equation,

$$
R T_{1} T_{2}=T_{2} T_{1} R
$$


Such an $R$-matrix satisifies the equation,

$$
R_{12} R_{13} R_{23}=R_{23} R_{13} R_{12}
$$

called the quantum Yang-Baxter equation ( $Q Y B E)$. The notion of a quantum group, a deformation of either the algebra of functions on a Lie group or the universal enveloping algebra of the associated Lie algebra, evolved from these considerations.

Just as quantum $R$-matrices and quantum groups play an important role in QISM, their classical limits, classiical $r$-matrices and Poisson Lie groups enter into the theory of classical integrable systems.

Poisson Lie groups are Lie groups equipped with an additional structure, a Poisson bracket satisfying a compatibility condition with the group multiplication. The infinitesimal object associated with a Poisson Lie group is the tangent vector space at the origin of the group, which is, in a natural way, a Lie algebra, $\mathfrak{g}$. The Poisson structure on the group induces on the Lie algebra an additional structure, which is nothing but a Lie algebra structure on the dual vector space $\mathfrak{g}^{*}$ satisfying a compatibility condition with the Lie bracket on $\mathfrak{g}$ itself. Such a Lie algebra together with its additional structure is called a Lie bialgebra. In most applications, the group is a group of matrices, while its Lie algebra is also an algebra of matrices of the same size, say $p \times p$. The classical $r$-matrices are then matrices of size $p^{2} \times p^{2}$. What is the relationship between such a matrix and the notion of bialgebra? The answer involves taking the Lie-algebra coboundary of the $r$-matrix (see Sect. 2.1). Another way to explain this relationship is as follows: Assume that we can identify the Lie algebra with its dual vector space by means of an invariant scalar product. Considering a Lie algebra structure on the dual vector space then amounts to considering a second Lie algebra structure on $\mathfrak{g}$ itself. When the $p^{2} \times p^{2} r$-matrix is identified with a linear map from $\mathfrak{g}$ to itself, which we denote by $R$, the second Lie bracket is given by

$$
[x, y]_{R}=[R x, y]+[x, R y]
$$

The modified Yang-Baxter equation (MYBE) is a sufficient condition for $R$ to define a second Lie bracket on $\mathfrak{g}$ by this formula, while the classical Yang-Baxter equation ( $C Y B E)$,

$$
\left[r_{12}, r_{13}\right]+\left[r_{12}, r_{23}\right]+\left[r_{13}, r_{23}\right]=0
$$

is the $r$-matrix version of this condition and is in fact obtained as a limit of the quantum Yang-Baxter equation.

In these lectures, we have tried to give a self-contained account of the theory of Lie bialgebras and Poisson Lie groups, including the basic definitions concerning the adjoint and coadjoint representations, Lie-algebra cohomology, Poisson manifolds and the Lie-Poisson structure of the dual of a Lie algebra, to explain all notations, including the 'tensor notation' which is ubiquitous in the 
physics literature, and to present the proofs of all the results. We have included the definitions of Manin triples, coboundary Lie bialgebras (triangular, quasitriangular and factorizable $r$-matrices), as well as the corresponding notions for Poisson Lie groups.

The examples that we discuss in detail are elementary, so we refer to the literature for a wealth of further examples.

Among the properties of Lie bialgebras and Lie groups, the existence of the dual and of the double of a Lie bialgebra, the integration theorem of a Lie bialgebra into a Poisson Lie group, whence the existence of the dual and of the double of a Poisson Lie group, are the most important. In the proofs, we use the Schouten bracket, whose importance in this theory was first pointed out by Gelfand and Dorfman [17] and emphasized by Magri and myself [38] [39] [40].

Semenov-Tian-Shansky's theorem, generalizing the theorem of Adler-KostantSymes, has important applications to the theory of integrable Hamiltonain systems. It establishes that, when a Lie algebra with an invariant scalar product is equipped with an $R$-matrix, the dynamical systems defined by an invariant function are in Lax form, and possess conserved quantities in involution (see Sect. 3.6). Whenever the original Lie algebra splits as a direct sum of two Lie subalgebras, the difference of the projections onto the Lie subalgebras (or a scalar multiple of this difference) is an $R$-matrix. (This occurs in many cases. The infinite-dimensional examples are the most interesting for the applications, but require some extension of the theory presented here. See [27] [28].) In this situation, such Lax equations can be solved by factorization, a method which replaces an initial-value problem with a problem of factorization in the associated Poisson Lie group. This is the reason for the name "factorizable $R$-matrix" given to the solutions of the modified Yang-Baxter equation. Thus, the $R$-matrix formalism can be considered to be an infinitesimal version of the Riemann-Hilbert factorization problem.

An essential ingredient of this theory and its applications to integrable systems is the notion of a Poisson action (of a Poisson Lie group on a Poisson manifold). It is a new concept which reduces to that of a Hamiltonian action when the Poisson structure on the Lie group vanishes. It was necessary to introduce such a generalization of Hamiltonian actions in order to account for the properties of the dressing transformations, under the "hidden symmetry group", of fields satisfying a zero-curvature equation. There are naturally defined actions of any Poisson Lie group on the dual Lie group, and conversely, and these are Poisson actions. (We give a one-line proof of this fact in Appendix 2, using the Poisson calculus.) In the case of a Poisson Lie group defined by a factorizable $R$-matrix, the explicit formulæ for these dressing actions coincide with the dressing of fields that are solutions of zero-curvature equations. There is a notion of momentum mapping for Poisson actions, and in this case it coincides with the monodromy matrix of the linear system. This establishes the connection between soliton equations which admit a zero-curvature representation 
(the compatibility condition for an auxiliary linear problem) in which the wave function takes values in a group and the theory of Poisson Lie groups.

In the bibliography, we have given references to

A. a variety of books from which all the prerequisites for a study of these lectures, and much more, can be learnt,

B. some of the articles that founded the subject, Drinfeld [15] [16], Gelfand and Dorfman [17], Semenov-Tian-Shansky [18] [19].

C. later expositions in books and surveys, e.g., Reyman and Semenov-TianShansky [27], Chari and Pressley [22], Vaisman [30], Reyman [26] and, for a survey of the Lie-algebraic approach to integrable systems, Perelomov [25].

D. a few of the most relevant publications that have further developed the subject, foremost among which is the article by Lu and Weinstein [44].

These lecture notes are meant as the necessary background for the survey of "Quantum and classical integrable systems" by Semenov-Tian-Shansky in this volume, ${ }^{1}$ where he briefly recalls the notions and results that we explain here, and some of their generalizations, and uses them extensively in the study of classical integrable systems, before studying their quantum counterpart.

These notes have been revised for clarity, and misprints have been corrected for the second edition of this book.

Acknowledgments. I gratefully acknowledge the support of CIMPA and of CEFIPRA-Indo-French Center for the Promotion of Advanced Research which permitted my stay at Pondicherry University in 1996 when I delivered the six lectures from which this text has been drawn.

\footnotetext{
${ }^{1}$ Remark on notations and conventions. Throughout these lectures, we reserve the term $r$-matrix on $\mathfrak{g}$ for elements of $\mathfrak{g} \otimes \mathfrak{g}$, and the term $R$-matrix for endomorphisms of $\mathfrak{g}$, while Semenov-Tian-Shansky uses the same letter $r$, and the term $r$-matrix, in both cases. In any case, these classical $r$-matrices and $R$-matrices should not be confused with the quantum $R$-matrices satisfying the quantum Yang-Baxter equation.

The bracket that we have associated here with a given $R$-matrix is twice the one which is defined in Semenov-Tian-Shansky's lectures. However, in the important special case where the second bracket is obtained as a result of the splitting of a Lie algebra into complementary Lie subalgebras, this bracket coincides with the bracket in his lectures, because the $R$-matrix that we consider is one-half of the difference of the projections, while he considers the difference of the projections.
} 


\section{Lie bialgebras}

We shall study Lie algebras $\mathfrak{g}$ whose dual vector space $\mathfrak{g}^{*}$ carries a Lie-algebra structure satisfying a compatibility condition, to be described in Sect. 1.3, with that of $\mathfrak{g}$ itself. Such objects are called Lie bialgebras. The corresponding Lie groups carry a Poisson structure compatible with the group multiplication (see Sect. 3 and 4). They constitute the semi-classical limit of quantum groups. In this section we shall study the general, abstract framework, and in Sect. 2 we shall describe the Lie-bialgebra structures defined by $r$-matrices, i.e., solutions of the classical Yang-Baxter equation.

\subsection{An example: $\mathfrak{s l}(2, \mathbb{C})$}

Let us consider the Lie algebra $\mathfrak{g}=\mathfrak{s l}(2, \mathbb{C})$, with basis

$$
H=\left(\begin{array}{cc}
1 & 0 \\
0 & -1
\end{array}\right), X=\left(\begin{array}{ll}
0 & 1 \\
0 & 0
\end{array}\right), Y=\left(\begin{array}{ll}
0 & 0 \\
1 & 0
\end{array}\right)
$$

and commutation relations,

$$
[H, X]=2 X, \quad[H, Y]=-2 Y, \quad[X, Y]=H .
$$

The dual vector space $\mathfrak{g}^{*}$ has the dual basis $H^{*}, X^{*}, Y^{*}$, where, by definition, $\left\langle H^{*}, H\right\rangle=1, \quad\left\langle X^{*}, X\right\rangle=1,\left\langle Y^{*}, Y\right\rangle=1$, and all other duality brackets are 0 . We shall consider the following commutation relations on $\mathfrak{g}^{*}$,

$$
\left[H^{*}, X^{*}\right]_{\mathfrak{g}^{*}}=\frac{1}{4} X^{*}, \quad\left[H^{*}, Y^{*}\right]_{\mathfrak{g}^{*}}=\frac{1}{4} Y^{*}, \quad\left[X^{*}, Y^{*}\right]_{\mathfrak{g}^{*}}=0 .
$$

Now consider $\mathfrak{g} \oplus \mathfrak{g}^{*}$. We can turn $\mathfrak{g} \oplus \mathfrak{g}^{*}$ into a Lie algebra, denoted by $\mathfrak{g} \bowtie \mathfrak{g}^{*}$ or $\mathfrak{d}$, and called the double of $\mathfrak{g}$, such that both $\mathfrak{g}$ and $\mathfrak{g}^{*}$ are Lie subalgebras of $\mathfrak{d}$, by setting

$$
\begin{aligned}
& {\left[H, H^{*}\right]_{\mathfrak{d}}=0, \quad\left[H, X^{*}\right]_{\mathfrak{d}}=-2 X^{*}, \quad\left[H, Y^{*}\right]_{\mathfrak{d}}=2 Y^{*}} \\
& {\left[X, H^{*}\right]_{\mathfrak{d}}=\frac{1}{4} X-Y^{*}, \quad\left[X, X^{*}\right]_{\mathfrak{d}}=-\frac{1}{4} H+2 H^{*}, \quad\left[X, Y^{*}\right]_{\mathfrak{d}}=0} \\
& {\left[Y, H^{*}\right]_{\mathfrak{d}}=\frac{1}{4} Y+X^{*}, \quad\left[Y, X^{*}\right]_{\mathfrak{d}}=0, \quad\left[Y, Y^{*}\right]_{\mathfrak{d}}=-\frac{1}{4} H-2 H^{*} .}
\end{aligned}
$$

We can prove the following facts :

(i) This is a Lie-algebra bracket on $\mathfrak{d}$, since we can show that it satisfies the Jacobi identity.

(ii) There is a natural scalar product $(\mid)$ on $\mathfrak{d}$, defined by

$$
\left(H \mid H^{*}\right)=\left(X \mid X^{*}\right)=\left(Y \mid Y^{*}\right)=1,
$$

while all other scalar products of elements in the basis vanish. With respect to this scalar product, $\mathfrak{g}$ and $\mathfrak{g}^{*}$ are isotropic, because the definition of an isotropic subspace is that the scalar product vanishes on it. 
(iii) The scalar product is invariant for the Lie-algebra structure of $\mathfrak{d}$ defined above. Recall that a scalar product $(\mid)$ on a Lie algebra a with bracket $[$,$] is$ called invariant if, for any $u, v, w \in \mathfrak{a}$,

$$
([u, v] \mid w)=(u \mid[v, w]) .
$$

\section{$1.2 \quad$ Lie-algebra cohomology}

In order to formulate the definition and properties of Lie bialgebras in general, we shall need a few definitions from the theory of Lie-algebra cohomology.

Let $\mathfrak{g}$ be a Lie algebra over the field of complex or real numbers. When $M$ is the vector space of a representation $\rho$ of $\mathfrak{g}$, we say that $\mathfrak{g}$ acts on $M$, or that $M$ is a $\mathfrak{g}$-module. For $x \in \mathfrak{g}, a \in M$, we often denote $(\rho(x))(a)$ simply by $x . a$.

Examples. Any Lie algebra $\mathfrak{g}$ acts on itself by the adjoint representation, $a d: x \in \mathfrak{g} \mapsto a d_{x} \in$ End $\mathfrak{g}$, defined, for $y \in \mathfrak{g}$, by $a d_{x}(y)=[x, y]$.

More generally, $\mathfrak{g}$ acts on any tensor product of $\mathfrak{g}$ with itself in the following way. For decomposable elements, $y_{1} \otimes \cdots \otimes y_{p}$ in $\stackrel{p}{\otimes} \mathfrak{g}=\mathfrak{g} \otimes \cdots \otimes \mathfrak{g}$ ( $p$ times),

$$
\begin{gathered}
x \cdot\left(y_{1} \otimes \cdots \otimes y_{p}\right)=a d_{x}^{(p)}\left(y_{1} \otimes \cdots \otimes y_{p}\right) \\
=a d_{x} y_{1} \otimes y_{2} \otimes \cdots \otimes y_{p}+y_{1} \otimes a d_{x} y_{2} \otimes y_{3} \otimes \cdots \otimes y_{p}+\cdots \\
+y_{1} \otimes y_{2} \otimes \cdots \otimes y_{p-1} \otimes a d_{x} y_{p} .
\end{gathered}
$$

For example, for $p=2$,

$$
a d_{x}^{(2)}\left(y_{1} \otimes y_{2}\right)=a d_{x} y_{1} \otimes y_{2}+y_{1} \otimes a d_{x} y_{2}=\left[x, y_{1}\right] \otimes y_{2}+y_{1} \otimes\left[x, y_{2}\right] .
$$

Thus, denoting the identity map from $\mathfrak{g}$ to $\mathfrak{g}$ by 1 ,

$$
a d_{x}^{(2)}=a d_{x} \otimes 1+1 \otimes a d_{x} .
$$

Since, when $y \in \mathfrak{g}, a d_{x} y=[x, y]$, one often writes

$$
\left(a d_{x} \otimes 1+1 \otimes a d_{x}\right)(u)=[x \otimes 1+1 \otimes x, u]
$$

for $u \in \mathfrak{g} \otimes \mathfrak{g}$.

Now, let $\mathfrak{g}$ be a finite-dimensional Lie algebra, and let $\left(e_{1}, \cdots, e_{n}\right)$ be a basis of $\mathfrak{g}$. Using the Einstein summation convention, any element $b$ in $\mathfrak{g} \otimes \mathfrak{g}$ can be written, $b=b^{i j} e_{i} \otimes e_{j}$, and then

$$
a d_{x}^{(2)} b=b^{i j}\left(\left[x, e_{i}\right] \otimes e_{j}+e_{i} \otimes\left[x, e_{j}\right]\right) .
$$

We could expand this quantity further in terms of the structure constants of the Lie algebra $\mathfrak{g}$ and the components of $x$. 
Similarly, $\mathfrak{g}$ acts on the $p$-th exterior power of $\mathfrak{g}, \bigwedge^{p} \mathfrak{g}$, for any $p$. For example, for $p=2$,

$$
\begin{gathered}
x .\left(y_{1} \wedge y_{2}\right)=\left[x, y_{1}\right] \wedge y_{2}+y_{1} \wedge\left[x, y_{2}\right], \\
x .\left(\sum_{i<j} a^{i j} e_{i} \wedge e_{j}\right)=\sum_{i<j} a^{i j}\left(\left[x, e_{i}\right] \wedge e_{j}+e_{i} \wedge\left[x, e_{j}\right]\right) .
\end{gathered}
$$

Definition. For each nonegative integer $k$, the vector space of skew-symmetric $k$-linear mappings on $\mathfrak{g}$ with values in $M$, where $M$ is the vector space of a representation of $\mathfrak{g}$, is called the space of $k$-cochains on $\mathfrak{g}$ with values in $M$.

A 1-cochain on $\mathfrak{g}$ with values in $M$ is just a linear map from $\mathfrak{g}$ to $M$, while a 0 -cochain on $\mathfrak{g}$ with values in $M$ is an element of $M$.

We can now define the coboundary of a $k$-cochain $u$ on $\mathfrak{g}$ with values in $M$, denoted by $\delta u$. Since we shall need only the cases where $k=0$ or 1 , we shall first write the definition in these two cases,

$$
\begin{gathered}
k=0, u \in M, x \in \mathfrak{g}, \delta u(x)=x . u, \\
k=1, v: \mathfrak{g} \rightarrow M, x, y \in \mathfrak{g}, \delta v(x, y)=x \cdot v(y)-y . v(x)-v([x, y]) .
\end{gathered}
$$

We immediately observe that for any 0 -cochain $u$ on $\mathfrak{g}$ with values in $M$,

$$
\delta(\delta u)=0 .
$$

In fact, for $x, y \in \mathfrak{g}$,

$$
(\delta(\delta u))(x, y)=x .(y \cdot u)-y \cdot(x . u))-[x, y] . u,
$$

and this quantity vanishes identically because $x \mapsto \rho(x)$ is a representation of $\mathfrak{g}$ in $M$. More generally,

Definition. The coboundary of a $k$-cochain $u$ on $\mathfrak{g}$ with values in $M$ is the $(k+1)$-cochain, $\delta u$, with values in $M$ defined by

$$
\begin{gathered}
\delta u\left(x_{0}, x_{1}, \cdots, x_{k}\right)=\sum_{i=0}^{k}(-1)^{i} x_{i} \cdot\left(u\left(x_{0}, \cdots, \hat{x}_{i}, \cdots, x_{k}\right)\right) \\
+\sum_{i<j}(-1)^{i+j} u\left(\left[x_{i}, x_{j}\right], x_{0}, \cdots, \hat{x}_{i}, \cdots, \hat{x}_{j}, \cdots, x_{k}\right),
\end{gathered}
$$

for $x_{0}, x_{1}, \cdots, x_{k} \in \mathfrak{g}$, where $\hat{x}_{i}$ indicates that the element $x_{i}$ is omitted.

Proposition. The property $\delta(\delta u)=0$ is valid for any $k$-cochain $u, k \geq 0$.

This is a standard result, which generalizes the property proved above for $k=0$. 
Definition. A $k$-cochain $u$ is called $a k$-cocycle if $\delta u=0 . A k$-cochain $u(k \geq 1)$ is called a $k$-coboundary if there exists a $(k-1)$-cochain, $v$, such that $u=\delta v$.

By the proposition, any $k$-coboundary is a $k$-cocycle. By definition, the quotient of the vector space of $k$-cocycles by the vector space of $k$-coboundaries is called the $k$-th cohomology vector space of $\mathfrak{g}$, with values in $M$.

Remark. The 0 -cocyles of $\mathfrak{g}$ with values in $M$ are the invariant elements in $M$, i.e., the elements $u \in M$ such that $x . u=0$, for each $x \in \mathfrak{g}$.

\subsection{Definition of Lie bialgebras}

Let us now assume that $\mathfrak{g}$ is a Lie algebra and that $\gamma$ is a linear map from $\mathfrak{g}$ to $\mathfrak{g} \otimes \mathfrak{g}$ whose transpose we denote by ${ }^{t} \gamma: \mathfrak{g}^{*} \otimes \mathfrak{g}^{*} \rightarrow \mathfrak{g}^{*}$. (If $\mathfrak{g}$ is infinitedimensional, $\mathfrak{g}^{*} \otimes \mathfrak{g}^{*}$ is a subspace of $(\mathfrak{g} \otimes \mathfrak{g})^{*}$, and what we are considering is in fact the restriction of the transpose of $\gamma$.) Recall that a linear map on $\mathfrak{g}^{*} \otimes \mathfrak{g}^{*}$ can be identified with a bilinear map on $\mathfrak{g}^{*}$.

Definition. A Lie bialgebra is a Lie algebra $\mathfrak{g}$ with a linear map $\gamma: \mathfrak{g} \rightarrow \mathfrak{g} \otimes \mathfrak{g}$ such that

(i) ${ }^{t} \gamma: \mathfrak{g}^{*} \otimes \mathfrak{g}^{*} \rightarrow \mathfrak{g}^{*}$ defines a Lie bracket on $\mathfrak{g}^{*}$, i.e., is a skew-symmetric bilinear map on $\mathfrak{g}^{*}$ satisfying the Jacobi identity, and

(ii) $\gamma$ is a 1-cocycle on $\mathfrak{g}$ with values in $\mathfrak{g} \otimes \mathfrak{g}$, where $\mathfrak{g}$ acts on $\mathfrak{g} \otimes \mathfrak{g}$ by the adjoint representation $a d^{(2)}$.

Condition (ii) means that the 2 -cochain $\delta \gamma$ vanishes, i.e., for $x, y \in \mathfrak{g}$,

$$
a d_{x}^{(2)}(\gamma(y))-a d_{y}^{(2)}(\gamma(x))-\gamma([x, y])=0 .
$$

Let us introduce the notation

$$
[\xi, \eta]_{\mathfrak{g}^{*}}={ }^{t} \gamma(\xi \otimes \eta),
$$

for $\xi, \eta \in \mathfrak{g}^{*}$. Thus, by definition, for $x \in \mathfrak{g}$,

$$
\left\langle[\xi, \eta]_{\mathfrak{g}^{*}}, x\right\rangle=\langle\gamma(x), \xi \otimes \eta\rangle .
$$

Condition $(i)$ is equivalent to the following,

$$
\left\{\begin{array}{l}
{[\xi, \eta]_{\mathfrak{g}^{*}}=-[\eta, \xi]_{\mathfrak{g}^{*}},} \\
{\left[\xi,[\eta, \zeta]_{\mathfrak{g}^{*}}\right]_{\mathfrak{g}^{*}}+\left[\eta,[\zeta, \xi]_{\mathfrak{g}^{*}}\right]_{\mathfrak{g}^{*}}+\left[\zeta,[\xi, \eta]_{\mathfrak{g}^{*}}\right]_{\mathfrak{g}^{*}}=0,}
\end{array}\right.
$$

An alternate way of writing condition $\left(i i^{\prime}\right)$ is

$$
\begin{aligned}
\left\langle[\xi, \eta]_{\mathfrak{g}^{*}},[x, y]\right\rangle & =\left\langle\xi \otimes \eta,\left(a d_{x} \otimes 1+1 \otimes a d_{x}\right)(\gamma(y))\right\rangle \\
& -\left\langle\xi \otimes \eta,\left(a d_{y} \otimes 1+1 \otimes a d_{y}\right)(\gamma(x))\right\rangle .
\end{aligned}
$$

(Recall that, by definition, $\left.\left(a d_{x} \otimes 1\right)\left(y_{1} \otimes y_{2}\right)=\left[x, y_{1}\right] \otimes y_{2}.\right)$ 


\subsection{The coadjoint representation}

We now introduce the important definition of the coadjoint representation of a Lie algebra on the dual vector space.

Let $\mathfrak{g}$ be a Lie algebra and let $\mathfrak{g}^{*}$ be its dual vector space. For simplicity, we shall assume that $\mathfrak{g}$ is finite-dimensional. For $x \in \mathfrak{g}$, we set

$$
a d_{x}^{*}=-{ }^{t}\left(a d_{x}\right) .
$$

Thus $a d_{x}^{*}$ is the endomorphism of $\mathfrak{g}^{*}$ satisfying

$$
\left\langle\xi, a d_{x} y\right\rangle=-\left\langle a d_{x}^{*} \xi, y\right\rangle
$$

for $y \in \mathfrak{g}, \xi \in \mathfrak{g}^{*}$. Then, it is easy to prove that the map $x \in \mathfrak{g} \mapsto a d_{x}^{*} \in$ End $\mathfrak{g}^{*}$ is a representation of $\mathfrak{g}$ in $\mathfrak{g}^{*}$.

Definition. The representation $x \mapsto a d_{x}^{*}$ of $\mathfrak{g}$ in $\mathfrak{g}^{*}$ is called the coadjoint representation of $\mathfrak{g}$.

\subsection{The dual of a Lie bialgebra}

In the notation of the preceding section, $\left(i i^{\prime}\right)$ of Section 1.3 can be written

$$
\begin{gathered}
\left\langle[\xi, \eta]_{\mathfrak{g}^{*}},[x, y]\right\rangle+\left\langle\left[a d_{x}^{*} \xi, \eta\right]_{\mathfrak{g}^{*}}, y\right\rangle+\left\langle\left[\xi, a d_{x}^{*} \eta\right]_{\mathfrak{g}^{*}}, y\right\rangle \\
-\left\langle\left[a d_{y}^{*} \xi, \eta\right]_{\mathfrak{g}^{*}}, x\right\rangle-\left\langle\left[\xi, a d_{y}^{*} \eta\right]_{\mathfrak{g}^{*}}, x\right\rangle=0 .
\end{gathered}
$$

We now see that there is a symmetry between $\mathfrak{g}$ with its Lie bracket $[$,$] and \mathfrak{g}^{*}$ with its Lie bracket $[,]_{\mathfrak{g}^{*}}$ defined by $\gamma$. In the same fashion as above, let us set

$$
\operatorname{ad}_{\xi} \eta=[\xi, \eta]_{\mathfrak{g}^{*}}
$$

and

$$
\left\langle a d_{\xi} \eta, x\right\rangle=-\left\langle\eta, a d_{\xi}^{*} x\right\rangle
$$

for $\xi, \eta \in \mathfrak{g}^{*}, x \in \mathfrak{g}$. Then $\xi \in \mathfrak{g}^{*} \mapsto a d_{\xi}^{*} \in$ End $\mathfrak{g}$ is the coadjoint representation of $\mathfrak{g}^{*}$ in the dual of $\mathfrak{g}^{*}$ which is isomorphic to $\mathfrak{g}$.

Now relation (ii) of Sect. 1.3 is equivalent to

$$
\begin{gathered}
\left\langle[\xi, \eta]_{\mathfrak{g}^{*}},[x, y]\right\rangle+\left\langle a d_{x}^{*} \xi, a d_{\eta}^{*} y\right\rangle-\left\langle a d_{x}^{*} \eta, a d_{\xi}^{*} y\right\rangle \\
-\left\langle a d_{y}^{*} \xi, a d_{\eta}^{*} x\right\rangle+\left\langle a d_{y}^{*} \eta, a d_{\xi}^{*} x\right\rangle=0 .
\end{gathered}
$$

It is now obvious that $\mathfrak{g}$ and $\mathfrak{g}^{*}$ play symmetric roles. Let us call $\mu: \mathfrak{g} \otimes \mathfrak{g} \rightarrow \mathfrak{g}$ the skew-symmetric bilinear mapping on $\mathfrak{g}$ defining the Lie bracket of $\mathfrak{g}$. Transforming relation $\left(i i^{\prime \prime}\right)$ again, it is easy to see that it is equivalent to the condition that ${ }^{t} \mu: \mathfrak{g}^{*} \rightarrow \mathfrak{g}^{*} \otimes \mathfrak{g}^{*}$ be a 1 -cocycle on $\mathfrak{g}^{*}$ with values in $\mathfrak{g}^{*} \otimes \mathfrak{g}^{*}$, where $\mathfrak{g}^{*}$ acts on $\mathfrak{g}^{*} \otimes \mathfrak{g}^{*}$ by the adjoint action. In fact, since the left-hand side of $\left(i i^{\prime \prime}\right)$ is

$$
\left\langle{ }^{t} \mu[\xi, \eta]_{\mathfrak{g}^{*}}, x \otimes y\right\rangle-\left\langle\xi,\left[x, a d_{\eta}^{*} y\right]\right\rangle+\left\langle\eta,\left[x, a d_{\xi}^{*} y\right]\right\rangle
$$




$$
+\left\langle\xi,\left[y, a d_{\eta}^{*} x\right]\right\rangle-\left\langle\eta,\left[y, a d_{\xi}^{*} x\right]\right\rangle,
$$

condition (ii) is equivalent to

$$
\begin{gathered}
\left\langle{ }^{t} \mu[\xi, \eta]_{\mathfrak{g}^{*}}, x \otimes y\right\rangle+\left\langle\left(a d_{\eta} \otimes 1+1 \otimes a d_{\eta}\right)\left({ }^{t} \mu(\xi)\right), x \otimes y\right\rangle \\
-\left\langle\left(a d_{\xi} \otimes 1+1 \otimes a d_{\xi}\right)\left({ }^{t} \mu(\eta)\right), x \otimes y\right\rangle=0
\end{gathered}
$$

or

$$
a d_{\xi}^{(2)}\left(\left({ }^{t} \mu\right)(\eta)\right)-a d_{\eta}^{(2)}\left(\left({ }^{t} \mu\right)(\xi)\right)-\left({ }^{t} \mu\right)\left([\xi, \eta]_{\mathfrak{g}^{*}}\right)=0 .
$$

Therefore

Proposition. If $(\mathfrak{g}, \gamma)$ is a Lie bialgebra, and $\mu$ is the Lie bracket of $\mathfrak{g}$, then $\left(\mathfrak{g}^{*},{ }^{t} \mu\right)$ is a Lie bialgebra, where ${ }^{t} \gamma$ is the Lie bracket of $\mathfrak{g}^{*}$.

By definition $\left(\mathfrak{g}^{*},{ }^{t} \mu\right)$ is called the dual of Lie bialgebra $(\mathfrak{g}, \gamma)$. Thus each Lie bialgebra has a dual Lie bialgebra whose dual is the Lie bialgebra itself.

\subsection{The double of a Lie bialgebra. Manin triples.}

Proposition. Let $(\mathfrak{g}, \gamma)$ be a Lie bialgebra with dual $\left(\mathfrak{g}^{*},{ }^{t} \mu\right)$. There exists a unique Lie-algebra structure on the vector space $\mathfrak{g} \oplus \mathfrak{g}^{*}$ such that $\mathfrak{g}$ and $\mathfrak{g}^{*}$ are Lie subalgebras and that the natural scalar product on $\mathfrak{g} \oplus \mathfrak{g}^{*}$ is invariant.

Proof. The natural scalar product on $\mathfrak{g} \oplus \mathfrak{g}^{*}$ is defined by

$$
(x \mid y)=0,(\xi \mid \eta)=0,(x \mid \xi)=\langle\xi, x\rangle, \text { for } x, y \in \mathfrak{g}, \xi, \eta \in \mathfrak{g}^{*} .
$$

Let us denote by $[u, v]_{\mathfrak{o}}$ the Lie bracket of two elements $u, v$ in $\mathfrak{d}=\mathfrak{g} \oplus \mathfrak{g}^{*}$. By the invariance condition on the natural scalar product and by the fact that $\mathfrak{g}$ is a Lie subalgebra, we obtain

$$
\begin{aligned}
&\left(y \mid[x, \xi]_{\mathfrak{d}}\right)=\left([y, x]_{\mathfrak{d}} \mid \xi\right)=([y, x] \mid \xi) \\
&=\langle\xi,[y, x]\rangle=\left\langle a d_{x}^{*} \xi, y\right\rangle=\left(y \mid a d_{x}^{*} \xi\right),
\end{aligned}
$$

and similarly $\left(\eta \mid[x, \xi]_{\mathfrak{o}}\right)=-\left(\eta \mid a d_{\xi}^{*} x\right)$, which proves that $[x, \xi]_{\mathfrak{o}}=-a d_{\xi}^{*} x+a d_{x}^{*} \xi$. One must now prove that the formulæ

$$
\left\{\begin{array}{l}
{[x, y]_{\mathfrak{d}}=[x, y]} \\
{[x, \xi]_{\mathfrak{d}}=-a d_{\xi}^{*} x+a d_{x}^{*} \xi} \\
{[\xi, \eta]_{\mathfrak{d}}=[\xi, \eta]_{\mathfrak{g}^{*}}}
\end{array}\right.
$$

define a Lie-algebra structure on $\mathfrak{g} \oplus \mathfrak{g}^{*}$. The proof of the Jacobi identity uses conditions (i) and (ii) of the definition of a Lie bialgebra.

Definition. When $\mathfrak{g}$ is a Lie bialgebra, $\mathfrak{g} \oplus \mathfrak{g}^{*}$ equipped with the Lie bracket $[,]_{\mathfrak{o}}$ defined by (1.2) is called the double of $\mathfrak{g}$, and denoted $\mathfrak{g} \bowtie \mathfrak{g}^{*}$ or $\mathfrak{d}$. 
We described an example in Sect. 1.1.

Note that $\mathfrak{d}=\mathfrak{g} \bowtie \mathfrak{g}^{*}$ is also the double of $\mathfrak{g}^{*}$. In the Lie algebra $\mathfrak{d}$, the subspaces $\mathfrak{g}$ and $\mathfrak{g}^{*}$ are complementary Lie subalgebras, and both are isotropic, i.e., the scalar product vanishes on $\mathfrak{g}$ and on $\mathfrak{g}^{*}$. Thus we see that, for any Lie bialgebra $\mathfrak{g},\left(\mathfrak{d}, \mathfrak{g}, \mathfrak{g}^{*}\right)$ is an example of a Manin triple, defined as follows:

Definition. A Manin triple is a triple $(\mathfrak{p}, \mathfrak{a}, \mathfrak{b})$, where $\mathfrak{p}$ is a Lie algebra with an invariant, non-degenerate, symmetric bilinear form, and $\mathfrak{a}$ and $\mathfrak{b}$ are complementary isotropic Lie subalgebras.

In the finite-dimensional case, we can show that, conversely, when $(\mathfrak{p}, \mathfrak{a}, \mathfrak{b})$ is a Manin triple, $\mathfrak{a}$ has a Lie-bialgebra structure. Since $\mathfrak{a}$ and $\mathfrak{b}$ play symmetric roles, $\mathfrak{b}$ also has a Lie-bialgebra structure, and the Lie bialgebra $\mathfrak{b}$ can be identified with the dual of the Lie bialgebra $\mathfrak{a}$. Let $(\mid)$ be the given scalar product on $\mathfrak{p}$. To $b \in \mathfrak{b}$ we associate the 1 -form $\iota(b)$ on $\mathfrak{a}$ defined by $\iota(b)(a)=(a \mid b)$. The linear map $b \mapsto \iota(b)$ from $\mathfrak{b}$ to $\mathfrak{a}^{*}$ is injective. In fact, if $\iota(b)=0$, then $(a \mid b)=0$ for all $a \in \mathfrak{a}$, and therefore for all $a \in \mathfrak{p}$, since $\mathfrak{b}$ is isotropic and $\mathfrak{p}=\mathfrak{a} \oplus \mathfrak{b}$. By the non-degeneracy of the scalar product, we find that $b=0$. Counting dimensions, we see that $\mathfrak{b}$ is isomorphic to $\mathfrak{a}^{*}$. The Lie bracket on $\mathfrak{b}$ therefore defines a Lie bracket on $\mathfrak{a}^{*}$. To see that it defines a Lie-bialgebra structure on $\mathfrak{a}$, we use the Jacobi identity in $\mathfrak{p}$, and the invariance of the scalar product. Thus

Theorem. There is a one-to-one correspondence between finite-dimensional Lie bialgebras and finite-dimensional Manin triples.

For a short, conceptual proof of this theorem, see Appendix 1.

\subsection{Examples}

\subsubsection{Simple Lie algebras over $\mathbb{C}$.}

Let $\mathfrak{g}$ be a simple Lie algebra over $\mathbb{C}$, of rank $r$, with Cartan subalgebra $\mathfrak{h}$, and with positive (resp., negative) Borel subalgebra $\mathfrak{b}_{+}$(resp., $\mathfrak{b}_{-}$), generated by $\mathfrak{h}$ and positive (resp., negative) root vectors.

Set $\mathfrak{p}=\mathfrak{g} \oplus \mathfrak{g}$ (direct sum of Lie algebras); let $\mathfrak{p}_{1}$ be the diagonal subalgebra, and $\mathfrak{p}_{2}=\left\{(x, y) \in \mathfrak{b}_{-} \oplus \mathfrak{b}_{+} \mid \mathfrak{h}\right.$-components of $x$ and $y$ are opposite $\}$. Define the scalar product of $(x, y)$ and $\left(x^{\prime}, y^{\prime}\right)$ to be $-\frac{1}{2}\left(\left(x \mid x^{\prime}\right)_{\mathfrak{g}}-\left(y \mid y^{\prime}\right)_{\mathfrak{g}}\right)$ where $(\mid)_{\mathfrak{g}}$ is the Killing form of $\mathfrak{g}$. (The factor $-\frac{1}{2}$ is conventional.) Then $\left(\mathfrak{p}, \mathfrak{p}_{1}, \mathfrak{p}_{2}\right)$ is a Manin triple, and the Lie brackets thus defined in $\mathfrak{g}^{*}$ can be explicitly written in terms of Weyl generators, $\left(H_{j}, X_{j}, Y_{j}\right), j=1, \ldots, r$.

Let us illustrate this fact for $\mathfrak{g}=\mathfrak{s} l(2, \mathbb{C})$. The Killing form of $\mathfrak{g}$ is such that

$$
(H \mid H)_{\mathfrak{g}}=8,(X \mid Y)_{\mathfrak{g}}=4,
$$

and all other scalar products vanish. In this case $\mathfrak{b}_{+}\left(\right.$resp., $\left.\mathfrak{b}_{-}\right)$is generated by $H$ and $X$ (resp., $H$ and $Y$ ). A basis for $\mathfrak{p}_{1}$ is $e_{1}=(H, H), e_{2}=(X, X), e_{3}=$ 
$(Y, Y)$. A basis for $\mathfrak{p}_{2}$ is $f_{1}=(H,-H), f_{2}=(0, X), f_{3}=(Y, 0)$. We find that

$$
\left(e_{i} \mid e_{j}\right)=0,\left(f_{i} \mid f_{j}\right)=0,\left(e_{1} \mid f_{1}\right)=-8,\left(e_{3} \mid f_{2}\right)=2,\left(e_{2} \mid f_{3}\right)=-2,
$$

and that all other scalar products vanish. We now identitfy $\mathfrak{p}_{2}$ with $\mathfrak{p}_{1}^{*} \simeq \mathfrak{g}^{*}$ by means of the scalar product, and we denote this identification map by $\iota$. Then $\iota\left(f_{1}\right)=-8 H^{*}, \iota\left(f_{2}\right)=2 Y^{*}, \iota\left(f_{3}\right)=-2 X^{*}$. Now

$$
\begin{aligned}
& {\left[f_{1}, f_{2}\right]=(0,-[H, X])=-2(0, X)=-2 f_{2},} \\
& {\left[f_{1}, f_{3}\right]=([H, Y], 0)=-2(Y, 0)=-2 f_{3},} \\
& {\left[f_{2}, f_{3}\right]=0,}
\end{aligned}
$$

and therefore we recover the commutation relations for $\mathfrak{g}^{*}$ given in Sect. 1.1,

$$
\left[H^{*}, X^{*}\right]=\frac{1}{4} X^{*},\left[H^{*}, Y^{*}\right]=\frac{1}{4} Y^{*},\left[X^{*}, Y^{*}\right]=0 .
$$

As a consequence, we see that the double of the Lie bialgebra $\mathfrak{s l}(2, \mathbb{C})$ is isomorphic to $\mathfrak{s l}(2, \mathbb{C}) \oplus \mathfrak{s l}(2, \mathbb{C})$.

\subsubsection{Compact Lie algebras.}

Let $\mathfrak{g}$ be a simple complex Lie algebra of rank $r$, with Cartan subalgebra $\mathfrak{h}$ and Weyl basis $\left(H_{j}, X_{\alpha}, Y_{\alpha}\right), j=1, \cdots, r$, and $\alpha \in \Delta_{+}$, where $\Delta_{+}$is the set of positive roots. Then the real linear span of

$$
i H_{j}, X_{\alpha}-Y_{\alpha}, i\left(X_{\alpha}+Y_{\alpha}\right)
$$

is a real subalgebra of $\mathfrak{g}^{\mathbb{R}}$, i.e., of $\mathfrak{g}$ considered as a real Lie algebra, denoted by $\mathfrak{k}$ and called the compact form of $\mathfrak{g}$. The real linear span of $i H_{j}, j=1, \cdots, r$, is a Cartan subalgebra $\mathfrak{t}$ of $\mathfrak{k}$, and $\mathfrak{h}=\mathfrak{t} \oplus i \mathfrak{t}$. Let $\mathfrak{b}=i \mathfrak{t} \oplus \mathfrak{n}_{+}$, where $\mathfrak{n}_{+}$is the Lie subalgebra generated by $X_{\alpha}, \alpha \in \Delta_{+}$. Then $\mathfrak{b}$ is a solvable, real Lie subalgebra of $\mathfrak{g}^{\mathbb{R}}$, and

$$
\mathfrak{g}^{\mathbb{R}}=\mathfrak{k} \oplus \mathfrak{b} .
$$

Define the scalar product on $\mathfrak{g}^{\mathbb{R}},(\mid)_{\mathfrak{g}^{\mathbb{R}}}=\operatorname{Im}(\mid)_{\mathfrak{g}}$, where $(\mid)_{\mathfrak{g}}$ is the Killing form of $\mathfrak{g}$, and Im denotes the imaginary part of a complex number. Then $\left(\mathfrak{g}^{\mathbb{R}}, \mathfrak{k}, \mathfrak{b}\right)$ is a Manin triple. Therefore $\mathfrak{k}$ (resp., $\mathfrak{b}$ ) is a Lie bialgebra with dual $\mathfrak{b}$ (resp., $\mathfrak{k}$ ).

We derive this Lie-bialgebra structure explicitly for $\mathfrak{g}=\mathfrak{s l}(2, \mathbb{C})$, in which case $\mathfrak{k}=\mathfrak{s u}(2)$. Let

$$
e_{1}=i H, e_{2}=X-Y, e_{3}=i(X+Y), f_{1}=H, f_{2}=X, f_{3}=i X,
$$

where $H, X, Y$ are as in Sect. 1.1. Then $\left(e_{1}, e_{2}, e_{3}\right)$ is a basis of $\mathfrak{s u}(2)$, while $\left(f_{1}, f_{2}, f_{3}\right)$ is a basis of the Lie subalgebra $\mathfrak{b}$ of $\mathfrak{g}^{\mathbb{R}}$ of complex, upper triangular $2 \times 2$ matrices, with real diagonal and vanishing trace. Thus $\mathfrak{s l}(2, \mathbb{C})=\mathfrak{s u}(2) \oplus \mathfrak{b}$. We observe that 


$$
e_{1}=\left(\begin{array}{cc}
i & 0 \\
0 & -i
\end{array}\right), e_{2}=\left(\begin{array}{cc}
0 & 1 \\
-1 & 0
\end{array}\right), e_{3}=\left(\begin{array}{cc}
0 & i \\
i & 0
\end{array}\right)
$$

We now identify $\mathfrak{b}$ with $\mathfrak{s u}(2)^{*}$ by the mapping $\iota$, where

$$
\iota(Z)(T)=\operatorname{Im}(Z \mid T)_{\mathfrak{g}} .
$$

Then $\iota\left(f_{1}\right)=8 e_{1}^{*}, \iota\left(f_{2}\right)=4 e_{3}^{*}, \iota\left(f_{3}\right)=-4 e_{2}^{*}$, where $\left(e_{1}^{*}, e_{2}^{*}, e_{3}^{*}\right)$ is the basis dual to the basis $\left(e_{1}, e_{2}, e_{3}\right)$ of $\mathfrak{s u}(2)$. Since $\left[f_{1}, f_{2}\right]=2 f_{2},\left[f_{1}, f_{3}\right]=2 f_{3},\left[f_{2}, f_{3}\right]=0$, we find that

$$
\left[e_{1}^{*}, e_{2}^{*}\right]=\frac{1}{4} e_{2}^{*},\left[e_{1}^{*}, e_{3}^{*}\right]=\frac{1}{4} e_{3}^{*},\left[e_{2}^{*}, e_{3}^{*}\right]=0 .
$$

These are the commutation relations of $\mathfrak{s u}(2)^{*}$, which is a solvable Lie algebra. Had we chosen the scalar product $\operatorname{tr} X_{1} X_{2}$ on $\mathfrak{g}$ instead of the Killing form, we would have obtained, as the commutation relations of $\mathfrak{s u}(2)^{*}$,

$$
\left[e_{1}^{*}, e_{2}^{*}\right]=e_{2}^{*},\left[e_{1}^{*}, e_{3}^{*}\right]=e_{3}^{*},\left[e_{2}^{*}, e_{3}^{*}\right]=0 .
$$

Remark. Let $\gamma_{0}$ be the 1 -cocycle on $\mathfrak{k}$ with values in $\bigwedge^{2} \mathfrak{k}$ defining the above Lie-bialgebra structure of $\mathfrak{k}$. It can be shown (see Soibelman [49]) that the most general Lie-bialgebra structure on the compact Lie algebra $\mathfrak{k}$ is

$$
\gamma=\lambda \gamma_{0}+\delta u
$$

where $\lambda$ is a real constant and $u$ is an arbitrary element of $\bigwedge^{2} \mathfrak{t}$. (Recall that $\delta u$ was defined in Sect. 1.2.)

\subsubsection{Infinite-dimensional Lie bialgebras}

The construction given for simple Lie algebras is also valid for Kac-Moody algebras.

Let $\mathfrak{a}$ be a finite-dimensional simple Lie algebra over $\mathbb{C}$, and let $\mathfrak{p}=\mathfrak{a}\left(\left(u^{-1}\right)\right)$ be the Lie algebra of the $\mathfrak{a}$-valued Laurent series in $u^{-1}, \mathfrak{p}_{1}=\mathfrak{a}[u]$ the Lie subalgebra of $\mathfrak{a}$-valued polynomials in $u$, and $\mathfrak{p}_{2}=u^{-1} \mathfrak{a}\left[\left[u^{-1}\right]\right]$, the Lie algebra of $\mathfrak{a}$-valued formal series in $u^{-1}$, with no constant term. Given $f, g \in \mathfrak{p}$, we define their scalar product to be the coefficient of $u^{-1}$ in the scalar-valued Laurent series in $u^{-1}$ obtained by taking the scalar product of the coefficients by means of the Killing form of $\mathfrak{a}$.

Then $\left(\mathfrak{p}, \mathfrak{p}_{1}, \mathfrak{p}_{2}\right)$ is a Manin triple. The corresponding 1-cocycle $\gamma$ on $\mathfrak{p}_{1}=\mathfrak{a}[u]$ can be written as follows. Since $(\mathfrak{a} \otimes \mathfrak{a})[u, v] \simeq \mathfrak{a}[u] \otimes \mathfrak{a}[v]$, for any $f \in \mathfrak{p}_{1}, \gamma(f)$ is an $(\mathfrak{a} \otimes \mathfrak{a})$-valued polynomial in two variables that can be expressed in terms of $t$, the Killing form of $\mathfrak{a}$, viewed as an element of $\mathfrak{a} \otimes \mathfrak{a}$. (A priori, $t$ is an element of $\mathfrak{a}^{*} \otimes \mathfrak{a}^{*}$, but, by means of the Killing form itself, this twice covariant tensor can be mapped to a twice contravariant tensor.) In fact,

$$
(\gamma(f))(u, v)=\left(a d_{f(u)} \otimes 1+1 \otimes a d_{f(v)}\right) \frac{t}{u-v},
$$


where 1 is the identity map of $\mathfrak{a}$ onto itself. If $\left(I^{k}\right)$ is an orthonormal basis of $\mathfrak{a}$ with respect to the Killing form, then $t=\sum_{k} I^{k} \otimes I^{k}$, and

$$
(\gamma(f))(u, v)=\frac{1}{u-v} \sum_{k}\left(\left[f(u), I^{k}\right] \otimes I^{k}+I^{k} \otimes\left[f(v), I^{k}\right]\right) .
$$

\subsection{Bibliographical note}

For this section, see Drinfeld [15] [16], Chari and Pressley [22], Chapter 1 or Vaisman [30], Chapter 10. (See also Kosmann-Schwarzbach [38], Verdier [31].) There are summaries of results on simple Lie algebras in Perelomov [25] and in Chari and Pressley [22, Appendix A] (page 562, line 4 of A2, read $\pm a_{i j}$, and page 564. line 9 of $\mathrm{A} 6$, read $T_{i}\left(x_{j}^{-}\right)$). For Lie-bialgebra structures on compact Lie algebras, see $\mathrm{Lu}$ and Weinstein [45] and Soibelman [49]. For the infinitedimensional example in Sect. 1.7, see Drinfeld [16], Chari and Pressley [22].

\section{Classical Yang-Baxter equation and $r$-matrices}

In this section, we shall study the Lie-bialgebra structures on a Lie algebra $\mathfrak{g}$ defined by a cocycle $\delta r$ which is the coboundary of an element $r \in \mathfrak{g} \otimes \mathfrak{g}$. Such elements $r \in \mathfrak{g} \otimes \mathfrak{g}$ are called $r$-matrices. We shall show that the classical Yang-Baxter equation $(C Y B E)$ is a sufficient condition for $\delta r$ to define a Lie bracket on $\mathfrak{g}^{*}$. We shall also define triangular, quasi-triangular and factorizable Lie bialgebras, show that the double of any Lie bialgebra is a factorizable Lie bialgebra, and we shall study examples.

\subsection{When does $\delta r$ define a Lie-bialgebra structure on $\mathfrak{g}$ ?}

We already noted that a 1-cochain on $\mathfrak{g}$ with values in $\mathfrak{g} \otimes \mathfrak{g}$ which is the coboundary of a 0-cochain on $\mathfrak{g}$ with values in $\mathfrak{g} \otimes \mathfrak{g}$, i.e., of an element $r \in \mathfrak{g} \otimes \mathfrak{g}$, is necessarily a 1-cocycle. So, in order for $\gamma=\delta r$ to define a Lie-bialgebra structure, there remain two conditions:

(i) $\delta r$ must take values in $\bigwedge^{2} \mathfrak{g}$ (skew-symmetry of the bracket on $\mathfrak{g}^{*}$ defined by $\delta r$,

(ii) the Jacobi identity for the bracket on $\mathfrak{g}^{*}$ defined by $\delta r$ must be satisfied.

Let us denote by $a$ (resp., $s$ ) the skew-symmetric (resp., symmetric) part of $r$. Thus $r=a+s$, where $a \in \bigwedge^{2} \mathfrak{g}, s \in S^{2} \mathfrak{g}$.

Let us assume for simplicity that $\mathfrak{g}$ is finite-dimensional. To any element $r$ in $\mathfrak{g} \otimes \mathfrak{g}$, we associate the map $\underline{r}: \mathfrak{g}^{*} \rightarrow \mathfrak{g}$ defined by

$$
\underline{r}(\xi)(\eta)=r(\xi, \eta)
$$


for $\xi, \eta \in \mathfrak{g}^{*}$. Here an element $r$ in $\mathfrak{g} \otimes \mathfrak{g}$ is viewed as a bilinear form on $\mathfrak{g}^{*}$, and an element $\underline{r}(\xi)$ in $\mathfrak{g}$ is viewed as a linear form on $\mathfrak{g}^{*}$. Another notation for $\underline{r}(\xi)(\eta)$ is $\langle\eta, \underline{r} \xi\rangle$. Let ${ }^{t} \underline{r}: \mathfrak{g}^{*} \rightarrow \mathfrak{g}$ denote the transpose of the map $\underline{r}$. Then, by definition,

$$
\underline{a}=\frac{1}{2}\left(\underline{r}-{ }^{t} \underline{r}\right), \underline{s}=\frac{1}{2}\left(\underline{r}+{ }^{t} \underline{r}\right) .
$$

Now let $\gamma=\delta r$. Then, by definition,

$$
\gamma(x)=a d_{x}^{(2)} r=\left(a d_{x} \otimes 1+1 \otimes a d_{x}\right)(r),
$$

where 1 is the identity map of $\mathfrak{g}$ into itself. The right-hand side stands for

$$
r^{i j}\left(a d_{x} e_{i} \otimes e_{j}+e_{i} \otimes a d_{x} e_{j}\right)
$$

when $\left(e_{i}\right)$ is a basis of $\mathfrak{g}$ and $r=r^{i j} e_{i} \otimes e_{j}$. As we explained in Sect. 1.2, the following notation is also used,

$$
a d_{x}^{(2)}(r)=[x \otimes 1+1 \otimes x, r] .
$$

For $\xi, \eta \in \mathfrak{g}^{*}$, we have set $[\xi, \eta]_{\mathfrak{g}^{*}}={ }^{t} \gamma(\xi, \eta)$. When $\gamma=\delta r$, we shall write $[\xi, \eta]^{r}$ instead of $[\xi, \eta]_{\mathfrak{g}^{*}}$.

Condition $(i)$ above is satisfied if and only if $\delta s=0$, that is, $s$ is invariant under the adjoint action,

$$
a d_{x}^{(2)} s=0
$$

for all $x \in \mathfrak{g}$. This condition is often written $[x \otimes 1+1 \otimes x, s]=0$. We shall often make use of the equivalent form of the $a d$-invariance condition for $s$,

$$
a d_{x} \circ \underline{s}=\underline{s} \circ a d_{x}^{*}, \text { for all } x \in \mathfrak{g} .
$$

Whenever $s$ is $a d$-invariant, $\delta r=\delta a$, and conversely. These equivalent conditions are obviously satisfied when $s=0$, i.e., when $r$ is skew-symmetric $(r=a)$.

Proposition. When $r$ is skew-symmetric, then

$$
[\xi, \eta]^{r}=a d_{\underline{r} \xi}^{*} \eta-a d_{\underline{r} \eta}^{*} \xi .
$$

Proof. By the definition of $\delta r$ and that of the coadjoint action,

$$
\left\langle{ }^{t}(\delta r)(\xi, \eta), x\right\rangle=\left(\left(a d_{x} \otimes 1+1 \otimes a d_{x}\right)(r)\right)(\xi, \eta)=-r\left(a d_{x}^{*} \xi, \eta\right)-r\left(\xi, a d_{x}^{*} \eta\right) .
$$

By the skew-symmetry of $r$ and the definition of $\underline{r}$, we find that

$$
\begin{aligned}
& -r\left(a d_{x}^{*} \xi, \eta\right)-r\left(\xi, a d_{x}^{*} \eta\right)=r\left(\eta, a d_{x}^{*} \xi\right)-r\left(\xi, a d_{x}^{*} \eta\right) \\
= & \underline{r}(\eta)\left(a d_{x}^{*} \xi\right)-\underline{r}(\xi)\left(a d_{x}^{*} \eta\right)=\left\langle a d_{x}^{*} \xi, \underline{r} \eta\right\rangle-\left\langle a d_{x}^{*} \eta, \underline{r} \xi\right\rangle .
\end{aligned}
$$


Since for $x, y \in \mathfrak{g}, \alpha \in \mathfrak{g}^{*},\left\langle a d_{x}^{*} \alpha, y\right\rangle=-\langle\alpha,[x, y]\rangle=\langle\alpha,[y, x]\rangle$, we obtain the general and useful relation,

$$
\left\langle a d_{x}^{*} \alpha, y\right\rangle=-\left\langle a d_{y}^{*} \alpha, x\right\rangle .
$$

Whence,

$$
{ }^{t}(\delta r)(\xi, \eta)=a d_{\underline{r} \xi}^{*} \eta-a d_{\underline{\eta} \eta}^{*} \xi,
$$

and the proposition is proved.

We shall now study condition (ii). We introduce the algebraic Schouten bracket of an element $r \in \bigwedge^{2} \mathfrak{g}$ with itself, denoted by $\llbracket r, r \rrbracket$. It is the element in $\bigwedge^{3} \mathfrak{g}$ defined by

$$
\llbracket r, r \rrbracket(\xi, \eta, \zeta)=-2 \circlearrowleft\langle\zeta,[\underline{r} \xi, \underline{r} \eta]\rangle,
$$

where $\circlearrowleft$ denotes the summation over the circular permutations of $\xi, \eta, \zeta$. (The factor -2 is conventional.)

Proposition. A necessary and sufficient condition for $\gamma=\delta r, r \in \bigwedge^{2} \mathfrak{g}$, to define a Lie bracket on $\mathfrak{g}^{*}$ is that $\llbracket r, r \rrbracket \in \Lambda^{3} \mathfrak{g}$ be ad-invariant.

Proof. Here we give a computational proof. See Appendix 1 for a shorter, more conceptual proof. Note that the element $\llbracket r, r \rrbracket$ is a 0 -cochain on $\mathfrak{g}$ with values in $\bigwedge^{3} \mathfrak{g}$. It is $a d$-invariant if and only if $\delta(\llbracket r, r \rrbracket)=0$. The proposition will then follow from the identity

$$
\circlearrowleft\left\langle\left[[\xi, \eta]^{r}, \zeta\right]^{r}, x\right\rangle=\frac{1}{2} \delta(\llbracket r, r \rrbracket)(x)(\xi, \eta, \zeta),
$$

for $\xi, \eta, \zeta \in \mathfrak{g}^{*}, x \in \mathfrak{g}$.

By $(2.2)$,

$$
\begin{gathered}
\left\langle\left[[\xi, \eta]^{r}, \zeta\right]^{r}, x\right\rangle=\left\langle\left[a d_{\underline{\underline{r}} \xi}^{*} \eta-a d_{\underline{\underline{r}} \eta}^{*} \xi, \zeta\right]^{r}, x\right\rangle \\
=\left\langle a d_{\underline{\underline{r}}\left(a d_{\underline{\underline{r}} \xi}^{*} \eta-a d_{\underline{\underline{\eta}} \eta}^{*} \xi\right)}^{*} \zeta, x\right\rangle-\left\langle a d_{\underline{\underline{r}} \zeta}^{*}\left(a d_{\underline{\underline{r}} \xi}^{*} \eta-a d_{\underline{r} \eta}^{*} \xi\right), x\right\rangle .
\end{gathered}
$$

By (2.3), this expression is equal to

$$
-\left\langle a d_{x}^{*} \zeta, \underline{r}\left(a d_{\underline{r} \xi}^{*} \eta-a d_{\underline{r} \eta}^{*} \xi\right)\right\rangle+\left\langle a d_{x}^{*}\left(a d_{\underline{\underline{\xi}}}^{*} \eta-a d_{\underline{r} \eta}^{*} \xi\right), \underline{r} \zeta\right\rangle .
$$

Using the skew-symmetry of $r$ and the relation

$$
a d_{x}^{*} a d_{y}^{*}-a d_{y}^{*} a d_{x}^{*}=a d_{[x, y]}^{*},
$$

valid for any $x, y \in \mathfrak{g}$, applied to $y=\underline{r} \xi$, we obtain

$$
\begin{aligned}
\left\langle\left[[\xi, \eta]^{r}, \zeta\right]^{r}, x\right\rangle= & \left\langle\underline{r} a d_{x}^{*} \zeta, a d_{\underline{r} \xi}^{*} \eta\right\rangle-\left\langle\underline{r} a d_{x}^{*} \zeta, a d_{\underline{r} \eta}^{*} \xi\right\rangle+\left\langle a d_{\underline{r} \xi}^{*} a d_{x}^{*} \eta, \underline{r} \zeta\right\rangle \\
& +\left\langle a d_{[x, \underline{r} \xi]}^{*} \eta, \underline{r} \zeta\right\rangle-\left\langle a d_{x}^{*} a d_{\underline{r} \eta}^{*} \xi, \underline{r} \zeta\right\rangle .
\end{aligned}
$$


Using (2.3), we obtain $\left\langle a d_{[x, \underline{r} \xi]}^{*} \eta, \underline{r} \zeta\right\rangle=-\left\langle a d_{\underline{r} \zeta}^{*} \eta,[x, \underline{r} \xi]\right\rangle=\left\langle a d_{x}^{*} a d_{\underline{r} \zeta}^{*} \eta, \underline{r} \xi\right\rangle$. Therefore,

$$
\begin{aligned}
\circlearrowleft\left\langle\left[[\xi, \eta]^{r}, \zeta\right]^{r}, x\right\rangle & =\circlearrowleft\left(\left\langle\eta,\left[\underline{r} a d_{x}^{*} \zeta, \underline{r} \xi\right]\right\rangle+\left\langle\xi,\left[\underline{r} \eta, \underline{r} a d_{x}^{*} \zeta\right]\right\rangle+\left\langle a d_{x}^{*} \eta,[\underline{r} \zeta, \underline{r} \xi]\right\rangle\right) \\
& +\circlearrowleft\left(\left\langle a d_{x}^{*} a d_{\underline{r} \zeta}^{*} \eta, \underline{r} \xi\right\rangle-\left\langle a d_{x}^{*} a d_{\underline{r} \eta}^{*} \xi, \underline{r} \zeta\right\rangle\right) .
\end{aligned}
$$

The last summation obviously vanishes. Now, by (2.4),

$$
\begin{gathered}
\frac{1}{2} \delta(\llbracket r, r \rrbracket)(x)(\xi, \eta, \zeta)=\frac{1}{2} a d_{x}^{(3)} \llbracket r, r \rrbracket(\xi, \eta, \zeta)=-\frac{1}{2} \circlearrowleft \llbracket r, r \rrbracket\left(\xi, \eta, a d_{x}^{*} \zeta\right) \\
=\circlearrowleft\left(\left\langle a d_{x}^{*} \zeta,[\underline{r} \xi, \underline{r} \eta]\right\rangle+\left\langle\eta,\left[\underline{r} a d_{x}^{*} \zeta, \underline{r} \xi\right]\right\rangle+\left\langle\xi,\left[\underline{r} \eta, \underline{r} a d_{x}^{*} \zeta\right]\right\rangle\right) .
\end{gathered}
$$

Comparing the two expressions we have just found, we see that (2.5) is proved.

Let $r$ be a skew-symmetric element of $\mathfrak{g} \otimes \mathfrak{g}$. The condition that $\llbracket r, r \rrbracket$ be $a d$ invariant is sometimes called the generalized Yang-Baxter equation. Obviously, a sufficient condition for $\llbracket r, r \rrbracket$ to be $a d$-invariant is

$$
\llbracket r, r \rrbracket=0 .
$$

We shall see that condition (2.6) is a particular case of the classical Yang-Baxter equation. (See Sect. 2.2.)

Definition. Let $r$ be an element in $\mathfrak{g} \otimes \mathfrak{g}$, with symmetric part $s$, and skewsymmetric part $a$. If $s$ and $\llbracket a, a \rrbracket$ are ad-invariant, then $r$ is called a classical $r$-matrix or, if no confusion is possible, an $r$-matrix. If $r$ is skew-symmetric $(r=a)$ and if $\llbracket r, r \rrbracket=0$, then $r$ is called $a$ triangular $r$-matrix.

Remark 1. It follows from the preceding discussion that any $r$-matrix in $\mathfrak{g} \otimes \mathfrak{g}$ defines a Lie-bialgebra structure on $\mathfrak{g}$ for which the Lie bracket on $\mathfrak{g}^{*}$ is given by formula (2.2). This bracket is called the Sklyanin bracket defined by $r$.

Remark 2. Some authors (see Babelon and Viallet [35], Li and Parmentier [43], Reiman [26]) define an $r$-matrix to be an element $r$ in $\mathfrak{g} \otimes \mathfrak{g}$ such that $a d_{\underline{r} \xi}^{*} \eta-a d_{\underline{r} \eta}^{*} \xi$ is a Lie bracket. In this definition, $s$ is not necessarily $a d$-invariant, and such $\bar{a}$ Lie bracket is not, in general, a Lie-bialgebra bracket.

In Sect. 2.4 we shall study the modified Yang-Baxter equation and its solutions, which are called classical $R$-matrices, or when no confusion can arise, $R$-matrices.

\subsection{The classical Yang-Baxter equation}

Let $r$ be an element in $\mathfrak{g} \otimes \mathfrak{g}$, and let us introduce $\langle\underline{r, r}\rangle: \bigwedge^{2} \mathfrak{g}^{*} \rightarrow \mathfrak{g}$, defined by

$$
\langle\underline{r, r}\rangle(\xi, \eta)=[\underline{r} \xi, \underline{r} \eta]-\underline{r}[\xi, \eta]^{r} .
$$


Setting

$$
\langle r, r\rangle(\xi, \eta, \zeta)=\langle\zeta,\langle\underline{r, r}\rangle(\xi, \eta)\rangle
$$

the map $\langle\underline{r, r}\rangle$ is identified with an element $\langle r, r\rangle \in \bigwedge^{2} \mathfrak{g} \otimes \mathfrak{g}$. We shall show that, whenever the symmetric part of $r$ is $a d$-invariant, the element $\langle r, r\rangle$ is in fact in $\bigwedge^{3} \mathfrak{g}$.

Theorem. (i) Let $a$ be in $\mathfrak{g} \otimes \mathfrak{g}$ and skew-symmetric. Then $\langle a, a\rangle$ is in $\bigwedge^{3} \mathfrak{g}$, and

$$
\langle a, a\rangle=-\frac{1}{2} \llbracket a, a \rrbracket
$$

(ii) Let $s$ be in $\mathfrak{g} \otimes \mathfrak{g}$, symmetric and ad-invariant. Then $\langle s, s\rangle$ is an ad-invariant element in $\bigwedge^{3} \mathfrak{g}$, and

$$
\langle\underline{s, s}\rangle(\xi, \eta)=[\underline{s} \xi, \underline{s} \eta],
$$

(iii) For $r=a+s$, where $a$ is skew-symmetric, and $s$ is symmetric and adinvariant, $\langle r, r\rangle$ is in $\bigwedge^{3} \mathfrak{g}$, and

$$
\langle r, r\rangle=\langle a, a\rangle+\langle s, s\rangle
$$

\section{Proof.}

(i) By definition, $\langle a, a\rangle(\xi, \eta, \zeta)=\langle\zeta,[\underline{a} \xi, \underline{a} \eta]\rangle-\left\langle\zeta, \underline{a}[\xi, \eta]^{a}\right\rangle$. By (2.2) and the skew-symmetry of $\underline{a}$,

$$
\begin{gathered}
\langle a, a\rangle(\xi, \eta, \zeta)=\langle\zeta,[\underline{a} \xi, \underline{a} \eta]\rangle-\left\langle\zeta, \underline{a} a d_{\underline{a} \xi}^{*} \eta\right\rangle+\left\langle\zeta, \underline{a} a d_{\underline{a} \eta}^{*} \xi\right\rangle \\
=\langle\zeta,[\underline{a} \xi, \underline{a} \eta]\rangle+\left\langle a d_{\underline{a} \xi}^{*} \eta, \underline{a} \zeta\right\rangle-\left\langle a d_{\underline{a} \eta}^{*} \xi, \underline{a} \zeta\right\rangle \\
=\langle\zeta,[\underline{a} \xi, \underline{a} \eta]\rangle+\langle\eta,[\underline{a} \zeta, \underline{a} \xi]\rangle+\langle\xi,[\underline{a} \eta, \underline{a} \zeta]\rangle .
\end{gathered}
$$

By (2.4), we see that

$$
\langle a, a\rangle(\xi, \eta, \zeta)=-\frac{1}{2} \llbracket a, a \rrbracket(\xi, \eta, \zeta)
$$

and therefore $(i)$ is proved.

(ii) If $s$ is symmetric and $a d$-invariant, then clearly $\langle\underline{s, s}\rangle(\xi, \eta)=[\underline{s} \xi, \underline{s} \eta]$. To see that $\langle s, s\rangle$ is in $\bigwedge^{3} \mathfrak{g}$, we use the ad-invariance of $s$ again and the symmetry of $\underline{s}$. Since

$$
\langle s, s\rangle(\xi, \eta, \zeta)=\langle\zeta,[\underline{s} \xi, \underline{s} \eta]\rangle,
$$

we find that

$$
\langle s, s\rangle(\xi, \eta, \zeta)=\left\langle\zeta, a d_{\underline{s} \xi} \underline{s} \eta\right\rangle=\left\langle\zeta, \underline{s} a d_{\underline{s} \xi}^{*} \eta\right\rangle
$$




$$
=\left\langle a d_{\underline{s} \xi}^{*} \eta, \underline{s} \zeta\right\rangle=-\langle\eta,[\underline{s} \xi, \underline{s} \zeta]\rangle=-\langle s, s\rangle(\xi, \zeta, \eta) .
$$

Thus $\langle s, s\rangle$ is skew-symmetric in the last two variables, therefore $\langle s, s\rangle \in \Lambda^{3} \mathfrak{g}$.

To prove that $\langle s, s\rangle$ is $a d$-invariant, we must prove that

$$
\langle\underline{s, s}\rangle\left(a d_{x}^{*} \xi, \eta\right)+\langle\underline{s, s}\rangle\left(\xi, a d_{x}^{*} \eta\right)=a d_{x}(\langle\underline{\langle s, s}\rangle(\xi, \eta)) .
$$

Now

$$
\begin{gathered}
\langle\underline{s, s}\rangle\left(a d_{x}^{*} \xi, \eta\right)+\langle\underline{s, s}\rangle\left(\xi, a d_{x}^{*} \eta\right) \\
=\left[a d_{x} \underline{s} \xi, \underline{s} \eta\right]+\left[\xi, a d_{x} \underline{s} \eta\right]=a d_{x}[\underline{s} \xi, \underline{s} \eta]=a d_{x}(\langle\underline{s, s}\rangle(\xi, \eta)) .
\end{gathered}
$$

(iii) From the $a d$-invariance of $s$, we know that

$$
[\xi, \eta]^{r}=[\xi, \eta]^{a} .
$$

Thus, since $r=a+s$, we find that

$$
\begin{gathered}
\langle\underline{r, r}\rangle(\xi, \eta)=[\underline{a} \xi, \underline{a} \eta]-\underline{a}[\xi, \eta]^{a} \\
+[\underline{a} \xi, \underline{s} \eta]+[\underline{s} \xi, \underline{a} \eta]-\underline{s}[\xi, \eta]^{a}+[\underline{s} \xi, \underline{s} \eta] .
\end{gathered}
$$

By $(2.2)$ and the $a d$-invariance of $\underline{s}$,

$$
\underline{s}[\xi, \eta]^{a}=\underline{s} a d_{\underline{a} \xi}^{*} \eta-\underline{s} a d_{\underline{a} \eta}^{*} \xi=a d_{\underline{a} \xi} \underline{s} \eta-a d_{\underline{a}} \eta \underline{s} \xi,
$$

and therefore $[\underline{a} \xi, \underline{s} \eta]+[\underline{s} \xi, \underline{a} \eta]-\underline{s}[\xi, \eta]^{a}=0$. Therefore $\langle\underline{r, r}\rangle=\langle\underline{a, a}\rangle+\langle\underline{s, s}\rangle$, thus proving (iii), since we already know that $\langle a, a\rangle$ and $\overline{\langle s, s\rangle}$ are in $\Lambda^{3} \mathfrak{g}$.

It follows from the proof of $(i)$ that,

$$
\langle a, a\rangle(\xi, \eta, \zeta)=\circlearrowleft\langle\zeta,[\underline{a} \xi, \underline{a} \eta]\rangle,
$$

while, if $s$ is $a d$-invariant, it follows from (ii) that

$$
\langle s, s\rangle(\xi, \eta, \zeta)=\langle\zeta,[\underline{s} \xi, \underline{s} \eta]\rangle .
$$

(In this case there is no summation over the circular permutations of $\xi, \eta, \zeta$.)

From this theorem, we obtain immediately,

Corollary. Let $r \in \mathfrak{g} \otimes \mathfrak{g}, r=a+s$, where $s$ is symmetric and ad-invariant, and $a$ is skew-symmetric. A sufficient condition for $\llbracket a, a \rrbracket$ to be ad-invariant is

$$
\langle r, r\rangle=0 .
$$

Thus an element $r \in \mathfrak{g} \otimes \mathfrak{g}$ with ad-invariant symmetric part, satisfying $\langle r, r\rangle=0$ is an $r$-matrix. 
Definition. Condition (2.7), $\langle r, r\rangle=0$, is called the classical Yang-Baxter equation. An r-matrix satisfying the classical Yang-Baxter equation is called quasi-triangular. If, moreover, the symmetric part of $r$ is invertible, then $r$ is called factorizable.

Remark 1. When $r$ is skew-symmetric, equation (2.7) reduces to equation (2.6). Thus a triangular $r$-matrix is quasi-triangular but not factorizable.

Remark 2. Sometimes condition (2.7), written in the form $\langle a, a\rangle=-\langle s, s\rangle$ is called the modified Yang-Baxter equation (for $a$ ), and the term classical YangBaxter equation is reserved for the case where $r$ is skew-symmetric, i.e., for condition (2.6). The abbreviations $C Y B E$ and $M Y B E$ are commonly used.

Remark 3. It is clear from part (iii) of the theorem that if $r=a+s$, then ${ }^{t} r=-a+s$ satisfies

$$
\left\langle{ }^{t} r,{ }^{t} r\right\rangle=\langle r, r\rangle \text {. }
$$

Thus, whenever $r$ is a solution of the classical Yang-Baxter equation, so are ${ }^{t} r$ and $-{ }^{t} r=a-s$. The notations

$$
r_{+}=r, \quad r_{-}=-{ }^{t} r
$$

will be used in Sect. 2.4, 2.5 and 4.9 below.

Observe also that if $r$ is a solution of the $C Y B E$, then so is any scalar multiple of $r$. However, if $a \in \bigwedge^{2} \mathfrak{g}$ is a solution of the $M Y B E$ in the sense of Remark 2 above, $\langle a, a\rangle=-\langle s, s\rangle$, for a given $s \in \mathfrak{g} \otimes \mathfrak{g}$, then $-a$ satisfies the same equation, but an arbitrary scalar multiple of $a$ does not.

The following proposition is immediate from the definition of $\underline{\langle r, r\rangle}$.

Proposition. An r-matrix, $r$, is quasi-triangular if and only if $\underline{r}_{+}=\underline{a}+\underline{s}$ and $\underline{r}_{-}=\underline{a}-\underline{s}$ are Lie-algebra morphisms from $\left(\mathfrak{g}^{*},[,]^{r}\right)$ to $\mathfrak{g}$.

We now prove

Proposition. On a simple Lie algebra over $\mathbb{C}$, any Lie-bialgebra structure is defined by a quasi-triangular r-matrix.

Proof. First, the Lie-bialgebra structure of any semi-simple Lie algebra, $\mathfrak{g}$, is necessarily defined by an $r$-matrix. In fact, when $\mathfrak{g}$ is semi-simple, by Whitehead's lemma, any 1-cocycle is a coboundary, and any 1-cocycle $\gamma$ with values in $\bigwedge^{2} \mathfrak{g}$ is the coboundary of an element $a \in \bigwedge^{2} \mathfrak{g}$,

$$
\gamma=\delta a
$$

We know that $\langle a, a\rangle=-\frac{1}{2} \llbracket a, a \rrbracket$ is an $a d$-invariant element of $\bigwedge^{3} \mathfrak{g}$, because ${ }^{t} \gamma$ is a Lie bracket on $\mathfrak{g}^{*}$, so $a$ is an $r$-matrix.

Let $t$ be the Killing form of $\mathfrak{g}$. It is an invariant, non-degenerate, symmetric, bilinear form on $\mathfrak{g}$, and $\langle t, t\rangle$ is an ad-invariant element of $\bigwedge^{3} \mathfrak{g}$, by (ii) of the 
theorem. We now use the result (see Koszul [11]) that, in a simple Lie algebra over $\mathbb{C}$, the space of $a d$-invariant elements of $\Lambda^{3} \mathfrak{g}$ is 1-dimensional. Therefore there exists a complex number $\mu$ such that

$$
\langle a, a\rangle=-\mu^{2}\langle t, t\rangle .
$$

Now, $r=a+\mu t$ is in fact a quasi-triangular $r$-matrix and $\delta r=\delta a=\gamma$.

\subsection{Tensor notation}

We have already observed in Sect. 1.2 that, for $r \in \mathfrak{g} \otimes \mathfrak{g}$, there are various notations for $\delta r(x), x \in \mathfrak{g}$,

$$
\delta r(x)=\left(a d_{x} \otimes 1+1 \otimes a d_{x}\right)(r)=[x \otimes 1+1 \otimes x, r] .
$$

We now introduce a new notation, not to be confused with the usual indicial notation of the tensor calculus. If $r \in \mathfrak{g} \otimes \mathfrak{g}$, we define $r_{12}, r_{13}, r_{23}$ as elements in the third tensor power of the enveloping algebra of $\mathfrak{g}$ (an associative algebra with unit such that $[x, y]=x \cdot y-y \cdot x)$,

$$
\begin{aligned}
& r_{12}=r \otimes 1, \\
& r_{23}=1 \otimes r,
\end{aligned}
$$

and, if $r=\Sigma_{i} u_{i} \otimes v_{i}$, then $r_{13}=\Sigma_{i} u_{i} \otimes 1 \otimes v_{i}$, where 1 is the unit of the enveloping algebra of $\mathfrak{g}$. This notation is called the tensor notation.

In $\mathfrak{g} \otimes \mathfrak{g} \otimes \mathfrak{g}$, we now define

$$
\left[r_{12}, r_{13}\right]=\left[\Sigma_{i} u_{i} \otimes v_{i} \otimes 1, \Sigma_{j} u_{j} \otimes 1 \otimes v_{j}\right]=\Sigma_{i, j}\left[u_{i}, u_{j}\right] \otimes v_{i} \otimes v_{j},
$$

and, similarly,

$$
\begin{aligned}
& {\left[r_{12}, r_{23}\right]=\left[\Sigma_{i} u_{i} \otimes v_{i} \otimes 1, \Sigma_{j} 1 \otimes u_{j} \otimes v_{j}\right]=\Sigma_{i, j} u_{i} \otimes\left[v_{i}, u_{j}\right] \otimes v_{j},} \\
& {\left[r_{13}, r_{23}\right]=\left[\Sigma_{i} u_{i} \otimes 1 \otimes v_{i}, \Sigma_{j} 1 \otimes u_{j} \otimes v_{j}\right]=\Sigma_{i, j} u_{i} \otimes u_{j} \otimes\left[v_{i}, v_{j}\right] .}
\end{aligned}
$$

In these notations, if the symmetric part $s$ of $r$ is $a d$-invariant, then

$$
\langle r, r\rangle=\left[r_{12}, r_{13}\right]+\left[r_{12}, r_{23}\right]+\left[r_{13}, r_{23}\right],
$$

and

$$
\langle s, s\rangle=\left[s_{13}, s_{23}\right]=\left[s_{23}, s_{12}\right]=\left[s_{12}, s_{13}\right] .
$$

In fact,

$$
\begin{aligned}
& {\left[r_{12}, r_{13}\right](\xi, \eta, \zeta)=\left\langle\xi,\left[{ }^{t} \underline{r} \eta,{ }^{t} \underline{r} \zeta\right]\right\rangle} \\
& {\left[r_{12}, r_{13}\right](\xi, \eta, \zeta)=\left\langle\eta,\left[\underline{r} \xi,{ }^{t} \underline{r} \zeta\right]\right\rangle}
\end{aligned}
$$




$$
\left[r_{13}, r_{23}\right](\xi, \eta, \zeta)=\langle\zeta,[\underline{r} \xi, \underline{r} \eta]\rangle
$$

while

$$
\begin{gathered}
\langle r, r\rangle(\xi, \eta, \zeta)=\langle\zeta,[\underline{r} \xi, \underline{r} \eta]\rangle-\left\langle\zeta, \underline{r}\left(a d_{\underline{r} \xi}^{*} \eta+a d_{\underline{t}}^{*} \eta \xi\right)\right\rangle \\
=\langle\zeta,[\underline{r} \xi, \underline{r} \eta]\rangle+\left\langle\eta,\left[\underline{r} \xi,{ }^{t} \underline{r} \zeta\right]\right\rangle+\left\langle\xi,\left[{ }^{t} \underline{r} \eta,{ }^{t} \underline{r} \zeta\right]\right\rangle .
\end{gathered}
$$

(We have used the fact that $a d_{\underline{s} \xi}^{*} \eta+a d_{\underline{s} \eta}^{*} \xi=0$, because of the $a d$-invariance of $s$, and therefore

$$
\left.a d_{\underline{\underline{\xi}}}^{*} \eta+a d_{\underline{t} \eta} \eta \xi=a d_{\underline{a} \xi}^{*} \eta-a d_{\underline{a} \eta}^{*} \xi=[\xi, \eta]^{r} .\right)
$$

Thus (2.8) is proved. Another way to state the $a d$-invariance of $s$ is

$$
\langle\xi,[x, \underline{s} \eta]\rangle+\langle\eta,[x, \underline{s} \xi]\rangle=0,
$$

and (2.9) follows.

So, in tensor notation, the classical Yang-Baxter equation (2.7) reads

$$
\left[r_{12}, r_{13}\right]+\left[r_{12}, r_{23}\right]+\left[r_{13}, r_{23}\right]=0 .
$$

Example 1. Let $\mathfrak{g}$ be the Lie algebra of dimension 2 with basis $X, Y$ and commutation relation

$$
[X, Y]=X
$$

Then $r=X \wedge Y=X \otimes Y-Y \otimes X$ is a skew-symmetric solution of $C Y B E$, i.e., a triangular $r$-matrix. This fact can be proved using definition (2.4), or one can prove (2.10). Here, for example,

$$
\begin{gathered}
{\left[r_{12}, r_{13}\right]=[X \otimes Y \otimes 1-Y \otimes X \otimes 1, X \otimes 1 \otimes Y-Y \otimes 1 \otimes X]} \\
=-[X, Y] \otimes Y \otimes X-[Y, X] \otimes X \otimes Y=-X \otimes Y \otimes X+X \otimes X \otimes Y,
\end{gathered}
$$

and similarly

$$
\begin{aligned}
& {\left[r_{12}, r_{23}\right]=-X \otimes X \otimes Y+Y \otimes X \otimes X,} \\
& {\left[r_{13}, r_{23}\right]=X \otimes Y \otimes X-Y \otimes X \otimes X}
\end{aligned}
$$

so that $\langle r, r\rangle=0$.

Then $\delta r(X)=0, \delta r(Y)=-X \wedge Y$. In terms of the dual basis $X^{*}, Y^{*}$ of $\mathfrak{g}^{*}$, $\left[X^{*}, Y^{*}\right]^{r}=-Y^{*}$.

Example 2. On $\mathfrak{s l}(2, \mathbb{C})$, we consider the Casimir element, $t$ (i.e., the Killing form seen as an element in $\mathfrak{g} \otimes \mathfrak{g}$ ),

$$
t=\frac{1}{8} H \otimes H+\frac{1}{4}(X \otimes Y+Y \otimes X)
$$


and we set

$$
\begin{aligned}
& t_{0}=\frac{1}{8} H \otimes H, \\
& t_{+-}=\frac{1}{4} X \otimes Y .
\end{aligned}
$$

If we define

$$
r=t_{0}+2 t_{+-}=\frac{1}{8}(H \otimes H+4 X \otimes Y)
$$

then the symmetric part of $r$ is $t$, and the skew-symmetric part is $a=\frac{1}{4} X \wedge Y$, and $r$ is a factorizable $r$-matrix. Then

$$
\delta a(H)=0, \delta a(X)=\frac{1}{4} X \wedge H, \delta a(Y)=\frac{1}{4} Y \wedge H,
$$

or, in terms of the dual basis $H^{*}, X^{*}, Y^{*}$ of $\mathfrak{g}^{*}$,

$$
\left[H^{*}, X^{*}\right]^{r}=\frac{1}{4} X^{*},\left[H^{*}, Y^{*}\right]^{r}=\frac{1}{4} Y^{*},\left[X^{*}, Y^{*}\right]^{r}=0 .
$$

Thus the Lie-bialgebra structure of $\mathfrak{s l}(2, \mathbb{C})$ of Sect. 1.1 is defined by the factorizable $r$-matrix given above.

Example 3. On $\mathfrak{s l}(2, \mathbb{C})$, we consider $r=X \otimes H-H \otimes X$, which is a triangular $r$-matrix. Then $\delta r(X)=0, \delta r(Y)=2 Y \wedge X, \delta r(H)=X \wedge H$.

\section{$2.4 R$-matrices and double Lie algebras}

Let now $R$ be any linear map from $\mathfrak{g}$ to $\mathfrak{g}$. We define

$$
[x, y]_{R}=[R x, y]+[x, R y]
$$

We consider the skew-symmetric bilinear form $\langle R, R\rangle$ on $\mathfrak{g}$ with values in $\mathfrak{g}$ defined by

$$
\langle R, R\rangle(x, y)=[R x, R y]-R([R x, y]+[x, R y])+[x, y]
$$

for $x, y \in \mathfrak{g}$, and, more generally, we define $\langle R, R\rangle_{k}$, by

$$
\langle R, R\rangle_{k}(x, y)=[R x, R y]-R([R x, y]+[x, R y])+k^{2}[x, y]
$$

where $k$ is any scalar.

Let $c \in \bigwedge^{3} \mathfrak{g}$, and define $\underline{c}: \bigwedge^{2} \mathfrak{g}^{*} \rightarrow \mathfrak{g}$ by

$$
\langle\zeta, \underline{c}(\xi, \eta)\rangle=c(\xi, \eta, \zeta) \text {. }
$$

Then, for $u \in \mathfrak{g}$,

$$
\begin{aligned}
\left(a d_{u} c\right)(\xi, \eta, \zeta) & =-\left(c\left(a d_{u}^{*} \xi, \eta, \zeta\right)+c\left(\xi, a d_{u}^{*} \eta, \zeta\right)+c\left(\xi, \eta, a d_{u}^{*} \zeta\right)\right) \\
& =-\circlearrowleft c\left(\xi, \eta, a d_{u}^{*} \zeta\right)=-\circlearrowleft\left\langle a d_{u}^{*} \zeta, \underline{c}(\xi, \eta)\right\rangle
\end{aligned}
$$


where $\circlearrowleft$ denotes the sum over cyclic permutations in $\xi, \eta, \zeta$. We have thus proved

Lemma. The ad-invariance of $c$ is equivalent to the condition on $\underline{c}$,

$$
\circlearrowleft\langle\zeta,[u, \underline{c}(\xi, \eta)]\rangle=0,
$$

for all $\xi, \eta, \zeta \in \mathfrak{g}^{*}, u \in \mathfrak{g}$.

Proposition. The Jacobi identity for the bracket [, ] $]_{R}$ defined by (2.11) is satisfied if and only if

$$
\underset{x, y, z}{\circlearrowleft}[z,\langle R, R\rangle(x, y)]=0
$$

for all $x, y, z$ in $\mathfrak{g}$.

Proof. We can show by a direct computation, using the Jacobi identity for the bracket [, ] repeatedly, that

$$
\circlearrowleft\left[[x, y]_{R}, z\right]_{R}-\circlearrowleft[z,\langle R, R\rangle(x, y)]
$$

$=\circlearrowleft([R[R x, y], z]+[[R x, y], R z]+[R[x, R y], z]+[[x, R y], R z])-\circlearrowleft[z,\langle R, R\rangle(x, y)]$ vanishes identically.

Definition. Condition

$$
\langle R, R\rangle_{k}=0
$$

is called the modified Yang-Baxter equation (MYBE) with coefficient $k^{2}$.

An endomorphism $R$ of $\mathfrak{g}$ satisfying $\langle R, R\rangle_{k}=0$ for some scalar $k$ is called a classical $R$-matrix, or simply an $R$-matrix. It is called factorizable if $k$ is not equal to 0 .

Thus any $R$-matrix on $\mathfrak{g}$ defines a second Lie-algebra structure $[,]_{R}$ on $\mathfrak{g}$. For this reason, a Lie algebra with an $R$-matrix is called a double Lie algebra. (This definition is not to be confused with that of the double of a Lie bialgebra, given in Sect. 1.6.)

Let $\mathfrak{g}$ be a Lie algebra with an ad-invariant, non-degenerate, symmetric bilinear form defining a bijective linear map $\underline{s}$ from $\mathfrak{g}^{*}$ to $\mathfrak{g}$. A skew-symmetric endomorphism of $(\mathfrak{g}, s)$ is a linear map $R$ from $\mathfrak{g}$ to $\mathfrak{g}$ such that $R \circ \underline{s}: \mathfrak{g}^{*} \rightarrow \mathfrak{g}$ is skew-symmetric.

Proposition. Let $R$ be a skew-symmetric endomorphism of $(\mathfrak{g}, s)$, and set $r=a+s$, where $\underline{a}=R \circ \underline{s}$. Then

$$
[x, y]_{R}=\underline{s}\left[\underline{s}^{-1} x, \underline{s}^{-1} y\right]^{r}=\underline{s}\left[\underline{s}^{-1} x, \underline{s}^{-1} y\right]^{a},
$$


and

$$
\langle R, R\rangle(x, y)=\langle\underline{r, r}\rangle\left(\underline{s}^{-1} x, \underline{s}^{-1} y\right) .
$$

Proof. The relations between $r=a+s$ and $R$ are

$$
\left\{\begin{array}{l}
R=\underline{a}^{\prime} \circ \underline{s}^{-1}, \\
\underline{r}=(R+1) \circ \underline{s},
\end{array}\right.
$$

where 1 is the identity map from $\mathfrak{g}$ to $\mathfrak{g}$. By the $a d$-invariance of $\underline{s}$,

$$
\begin{gathered}
{[R x, y]+[x, R y]=a d_{(R \circ \underline{s}) \underline{s}^{-1} x} y-a d_{(R \circ \underline{s}) \underline{s}^{-1} y} x} \\
\left.=\underline{s}\left(a d_{\underline{a}\left(\underline{s}^{-1} x\right)} \underline{s}^{-1} y-a d_{\underline{a}}^{*} \underline{s}^{-1} y\right) \underline{s}^{-1} x\right) .
\end{gathered}
$$

This relation and (2.2) prove (2.17), and

$$
R([R x, y]+[x, R y])=\underline{a}\left[\underline{s}^{-1} x, \underline{s}^{-1} y\right]^{a} .
$$

Thus, by the theorem of Sect. 2.2,

$$
\begin{aligned}
\langle R, R\rangle(x, y) & =\left[\underline{a}\left(\underline{s}^{-1} x\right), \underline{a}\left(\underline{s}^{-1} y\right)\right]-\underline{a}\left[\underline{s}^{-1} x, \underline{s}^{-1} y\right]^{a}+\left[\underline{s}\left(\underline{s}^{-1} x\right), \underline{s}\left(\underline{s}^{-1} y\right)\right] \\
& =\langle a, a\rangle\left(\underline{s}^{-1} x, \underline{s}^{-1} y\right)+\langle\underline{s, s}\rangle\left(\underline{s}^{-1} x, \underline{s}^{-1} y\right) \\
& =\left\langle\underline{a+s, a+s\rangle}\left(\underline{s}^{-1} x, \underline{s}^{-1} y\right)\right.
\end{aligned}
$$

and this is equality (2.18).

More generally, setting $r_{k}=a+k s$, we find that

$$
\langle R, R\rangle_{k}(x, y)=\left\langle\underline{r_{k}, r_{k}}\right\rangle\left(\underline{s}^{-1} x, \underline{s}^{-1} y\right) .
$$

Proposition. Let $R$ be a skew-symmetric endomorphism of $(\mathfrak{g}, s)$. Then $R$ is an $R$-matrix (resp., a factorizable $R$-matrix) if and only if $r_{k}=(R+k 1) \circ \underline{s}$ is a quasi-triangular $r$-matrix (resp., a factorizable $r$-matrix), for some scalar $k$.

Proof. This follows from the preceding proposition.

In other words, $R=\underline{a} \circ \underline{s}^{-1}$ satisfies the $M Y B E$ with coefficient $k^{2}$ if and only if $r=a+k s$ satisfies the $C Y B E$. In the terminology of Remark 2 of Sect. $2.2, R$ is a solution of the $M Y B E$ with coefficient 1 if and only if $a$, defined by $\underline{a}=R \circ \underline{s}$ satisfies the $M Y B E$.

Applying the lemma to $c=\langle r, r\rangle$, and using the preceding proposition, we see that $r=a+s$ is an $r$-matrix with invertible symmetric part $s$ if and only if $R=\underline{a} \circ \underline{s}^{-1}$ satisfies the condition

$$
\underset{x, y, z}{\circlearrowleft}\left\langle\underline{s}^{-1} z,[u,\langle R, R\rangle(x, y)]\right\rangle=0
$$


for each $x, y, z, u \in \mathfrak{g}$. Using the $a d$-invariance of $s$, we find that this condition is equivalent to

$$
\underset{x, y, z}{\circlearrowleft}[z,\langle R, R\rangle(x, y)]=0 .
$$

This fact furnishes an alternate proof of the first proposition of this section.

Example. If $\mathfrak{g}=\mathfrak{a} \oplus \mathfrak{b}$, where both $\mathfrak{a}$ and $\mathfrak{b}$ are Lie subalgebras of $\mathfrak{g}$, then $R=\frac{1}{2}\left(p_{\mathfrak{a}}-p_{\mathfrak{b}}\right)$, where $p_{\mathfrak{a}}$ (resp., $\left.p_{\mathfrak{b}}\right)$ is the projection onto $\mathfrak{a}$ (resp., $\mathfrak{b}$ ) parallel to $\mathfrak{b}$ (resp., $\mathfrak{a}$ ), is a solution of the modified Yang-Baxter equation with coefficient $\frac{1}{4}$. The proof is straightforward. In this case, we find that

$$
[x, y]_{R}=\left[x_{\mathfrak{a}}, y_{\mathfrak{a}}\right]-\left[x_{\mathfrak{b}}, y_{\mathfrak{b}}\right] .
$$

As an example, we can consider $\mathfrak{g}=\mathfrak{s l}(n, \mathbb{R}), \mathfrak{a}$ the subalgebra of upper triangular matrices, and $\mathfrak{b}=\mathfrak{s o}(n)$.

Proposition. If $R$ is a solution of the modified Yang-Baxter equation with coefficient $k^{2}$, then

$$
R_{ \pm}=R \pm k 1
$$

are Lie-algebra morphisms from $\mathfrak{g}_{R}$ to $\mathfrak{g}$, where $\mathfrak{g}_{R}$ denotes $\mathfrak{g}$ equipped with the Lie bracket $[,]_{R}$ defined by (2.11).

Proof. In fact,

$$
\begin{gathered}
R_{ \pm}[x, y]_{R}-\left[R_{ \pm} x, R_{ \pm} y\right]=(R \pm k 1)([R x, y]+[x, R y])-[(R \pm k 1) x,(R \pm k 1) y] \\
=R([R x, y]+[x, R y])-[R x, R y]-k^{2}[x, y]=-\langle R, R\rangle_{k}(x, y) .
\end{gathered}
$$

Remark. When $R$ is a solution of the $M Y B E$ with coefficient 1 , and $\underline{s}$ is invertible, then $R_{ \pm}=\underline{r}_{ \pm} \circ \underline{s}^{-1}$, and it follows from (2.11) and (2.17) that

$$
R_{ \pm}[x, y]_{R}-\left[R_{ \pm} x, R_{ \pm} y\right]=\underline{r}_{ \pm}\left[\underline{s}^{-1} x, \underline{s}^{-1} y\right]^{r}-\left[\underline{r}_{ \pm}\left(\underline{s}^{-1} x\right), \underline{r}_{ \pm}\left(\underline{s}^{-1} y\right)\right]
$$

Therefore the morphism properties of $R_{ \pm}$can be deduced from those of $r_{ \pm}$ proved in Sect. 2.2.

As a consequence of the preceding proposition we obtain the following

Proposition. Let $R$ be an $R$-matrix satisfying $\langle R, R\rangle_{k}=0$. Then

$$
J: x \in \mathfrak{g}_{R} \mapsto\left(R_{+} x, R_{-} x\right) \in \mathfrak{g} \oplus \mathfrak{g}
$$

is an injective map which identifies $\mathfrak{g}_{R}$ with a Lie subalgebra of the direct sum of Lie algebras $\mathfrak{g} \oplus \mathfrak{g}$.

Proof. The linear map $J$ is clearly injective since $R_{+} x=R_{-} x=0$ implies $\left(R_{+}-R_{-}\right) x=0$, and thus $x=0$. Moreover,

$$
J\left([x, y]_{R}\right)=\left(R_{+}[x, y]_{R}, R_{-}[x, y]_{R}\right)=\left(\left[R_{+} x, R_{+} y\right],\left[R_{-} x, R_{-} y\right]\right) \in \mathfrak{g} \oplus \mathfrak{g},
$$

proving the proposition. 


\subsection{The double of a Lie bialgebra is a factorizable Lie bial- gebra}

Let $(\mathfrak{g}, \gamma)$ be any Lie bialgebra, and let $\mathfrak{d}$ be its double. Recall that, as a vector space, $\mathfrak{d}$ is just $\mathfrak{g} \oplus \mathfrak{g}^{*}$. There is a canonical $R$-matrix defined on the Lie algebra $\left(\mathfrak{d},[,]_{\mathfrak{d}}\right)$, namely

$$
R=\frac{1}{2}\left(p_{\mathfrak{g}^{*}}-p_{\mathfrak{g}}\right)
$$

Since $\mathfrak{g}$ and $\mathfrak{g}^{*}$ are Lie subalgebras of $\mathfrak{d}$, this endomorphism of $\mathfrak{d}$ is indeed a factorizable $R$-matrix, which satisfies the modified Yang-Baxter equation with coefficient $k^{2}=\frac{1}{4}$ (with respect to the Lie bracket of the double). So $\mathfrak{d}$ is a double Lie algebra, with

$$
[x+\xi, y+\eta]_{R}=-[x, y]+[\xi, \eta]
$$

Moreover $\mathfrak{d}=\mathfrak{g} \oplus \mathfrak{g}^{*}$ has a natural scalar product, ( | ), which defines a linear $\operatorname{map} \underline{s}_{\mathfrak{d}}$ from $\mathfrak{d}^{*}=\mathfrak{g}^{*} \oplus \mathfrak{g}$ to $\mathfrak{d}$. It is easily seen that

$$
\underline{s}_{\mathfrak{d}}(\xi, x)=(x, \xi) .
$$

Note that $R$ is skew-symmetric with respect to this scalar product. Therefore, by the third proposition in Sect. 2.4, $\underline{r}_{\mathfrak{d}}=R \circ \underline{s}_{\mathfrak{d}}+\frac{1}{2} \underline{s}_{\mathfrak{d}}$ defines a factorizable $r$-matrix on $\mathfrak{d}$.

Explicitly, $\underline{r}_{\mathfrak{d}}$ is the linear map from $\mathfrak{d}^{*}$ to $\mathfrak{d}$ defined by

$$
\underline{r}_{\mathfrak{d}}(\xi, x)=(0, \xi),
$$

with symmetric part $\frac{1}{2} \underline{s}_{\mathfrak{d}}$ and skew-symmetric part

$$
\underline{a}_{\mathfrak{d}}(\xi, x)=\frac{1}{2}(-x, \xi)
$$

The bracket on $\mathfrak{d}^{*}$ defined by the $r$-matrix $r_{\mathfrak{d}}$ is

$$
[\xi+x, \eta+y]_{\mathfrak{d}^{*}}=[\xi, \eta]-[x, y] .
$$

(We use the notations $(\xi, x)$ or $\xi+x$ for elements of $\mathfrak{g}^{*} \oplus \mathfrak{g}$.) Thus in the Lie bialgebra $\mathfrak{d}$, the dual $\mathfrak{d}^{*}$ of $\mathfrak{d}$ is the direct sum of $\mathfrak{g}^{*}$ and the opposite of $\mathfrak{g}$.

Moreover, if the Lie bialgebra $\mathfrak{g}$ itself is quasi-triangular (the cocycle $\gamma$ is the coboundary of a quasi-triangular $r$-matrix), then the Lie algebra $\mathfrak{d}$ is a direct sum that is isomorphic to $\mathfrak{g} \oplus \mathfrak{g}$.

Proposition. If the Lie-bialgebra structure of $\mathfrak{g}$ is defined by a factorizable $r$-matrix, then its double is isomorphic to the direct sum of Lie algebras $\mathfrak{g} \oplus \mathfrak{g}$.

Proof. We first embed $\mathfrak{g}$ in $\mathfrak{g} \oplus \mathfrak{g}$ by $x \mapsto(x, x)$. Then we embed $\mathfrak{g}^{*}$ in $\mathfrak{g} \oplus \mathfrak{g}$ by $j: \xi \mapsto\left(\underline{r}_{+} \xi, \underline{r}_{-} \xi\right)$. This map $j$ is the map $J$ of the last proposition of Sect. 2.4 
composed with $\underline{s}: \mathfrak{g}^{*} \rightarrow \mathfrak{g}_{R}$, and formula (2.17) shows that it is a morphism of Lie algebras from $\mathfrak{g}^{*}$ to $\mathfrak{g} \oplus \mathfrak{g}$.

We obtain a Manin triple $\left(\mathfrak{g} \oplus \mathfrak{g}, \mathfrak{g}^{\text {diag }}, j\left(\mathfrak{g}^{*}\right)\right)$, where $\mathfrak{g}^{\text {diag }}$ is the diagonal subalgebra of $\mathfrak{g} \oplus \mathfrak{g}$, and $\mathfrak{g} \oplus \mathfrak{g}$ is equipped with the invariant scalar product

$$
\left((x, y),\left(x^{\prime}, y^{\prime}\right)\right)=\left\langle\underline{s} x, x^{\prime}\right\rangle-\left\langle\underline{s} y, y^{\prime}\right\rangle .
$$

This Manin triple is isomorphic to $\left(\mathfrak{d}, \mathfrak{g}, \mathfrak{g}^{*}\right)$.

Example. The double of $\mathfrak{g}=\mathfrak{s l}(2, \mathbb{R})$ is $\mathfrak{s l}(2, \mathbb{R}) \oplus \mathfrak{s l}(2, \mathbb{R})$. (This fact was proved directly in Sect. 1.7.) This property extends to $\mathfrak{s l}(n, \mathbb{R})$.

We shall see in Sect. 3.6 that Hamiltonian systems on double Lie algebras give rise to equations in Lax form, and in Sect. 4.9 that the double plays a fundamental role in the theory of dressing transformations.

\subsection{Bibliographical note}

This section is based on the articles by Drinfeld [15] [16] and Semenov-TianShansky [18] [19] [28]. See also Kosmann-Schwarzbach and Magri [40]. A survey and examples can be found in Chari and Pressley [22], Chapter 2. (Chapter 3 of [22] deals with the classification of the solutions of the classical Yang-Baxter equation, due to Belavin and Drinfeld, which we have not discussed in these lectures.) The first proposition in Sect. 2.1 is in [15] and, in invariant form, in [38] [40]. The proposition in Sect. 2.5 was proved independently by Aminou and Kosmann-Schwarzbach [33] and by Reshetikhin and Semenov-Tian-Shansky $[47]$.

\section{Poisson manifolds. The dual of a Lie algebra. Lax equations}

We shall now introduce Poisson manifolds and show that the dual of a finitedimensional Lie algebra $\mathfrak{g}$ is always a Poisson manifold.

In particular, when $\mathfrak{g}$ is a Lie bialgebra, we obtain a Poisson structure on $\mathfrak{g}$ itself from the Lie-algebra structure on $\mathfrak{g}^{*}$. We shall first express the Poisson brackets of functions on the dual $\mathfrak{g}^{*}$ of a Lie algebra $\mathfrak{g}$ in tensor notation and then show that, for a connected Lie group, the co-adjoint orbits in $\mathfrak{g}^{*}$ are the leaves of the symplectic foliation of $\mathfrak{g}^{*}$. Finally, we shall show that Hamiltonian systems on double Lie algebras give rise to equations in Lax form.

\subsection{Poisson manifolds}

On smooth manifolds, one can define Poisson structures, which give rise to Poisson brackets with the usual properties, on the space of smooth functions 
on the manifold in the following way. We shall henceforth write manifold for smooth manifold, tensor for smooth field of tensors, etc. Let us denote the space of functions on a manifold $M$ by $C^{\infty}(M)$. By a bivector on a manifold $M$ we mean a skew-symmetric, contravariant 2-tensor, i.e., if $P$ is a bivector, at each point $x \in M, P_{x}$ has skew-symmetric components in local coordinates, $\left(P^{i j}(x)\right), i, j=1,2, \cdots, \operatorname{dim} M$. At each point $x$, we can view $P_{x}$ as a skewsymmetric bilinear form on $T_{x}^{*} M$, the dual of the tangent space $T_{x} M$, or as the skew-symmetric linear map $\underline{P}_{x}$ from $T_{x}^{*} M$ to $T_{x} M$, such that

$$
\left\langle\eta_{x}, \underline{P}_{x}\left(\xi_{x}\right)\right\rangle=P_{x}\left(\xi_{x}, \eta_{x}\right), \text { for } \xi_{x}, \eta_{x} \in T_{x}^{*} M
$$

If $\xi, \eta$ are differential 1 -forms on $M$, we define $P(\xi, \eta)$ to be the function in $C^{\infty}(M)$ whose value at $x \in M$ is $P_{x}\left(\xi_{x}, \eta_{x}\right)$.

If $f, g$ are functions on $M$, and $d f, d g$ denote their differentials, we set

$$
\{f, g\}=P(d f, d g) .
$$

Note that $\underline{P}(d f)$ is a vector field, denoted by $X_{f}$, and that

$$
\{f, g\}=X_{f} \cdot g \text {. }
$$

It is clear that $\{f, g h\}=\{f, g\} h+g\{f, h\}$, for any functions $f, g, h$ on $M$, so that the bracket $\{$,$\} satisfies the Leibniz rule.$

Definition. A Poisson manifold $(M, P)$ is a manifold $M$ with a Poisson bivector $P$ such that the bracket defined by (3.2) satisfies the Jacobi identity.

When $(M, P)$ is a Poisson manifold, $\{f, g\}$ is called the Poisson bracket of $f$ and $g \in C^{\infty}(M)$, and $X_{f}=\underline{P}(d f)$ is called the Hamiltonian vector field with Hamiltonian $f$. Functions $f$ and $g$ are said to be in involution if $\{f, g\}=0$.

Example. If $M=\mathbb{R}^{2 n}$, with coordnates $\left(q^{i}, p_{i}\right), i=1, \cdots, n$, and if

$$
\underline{P}\left(d q^{i}\right)=-\frac{\partial}{\partial p_{i}}, \quad \underline{P}\left(d p_{i}\right)=\frac{\partial}{\partial q^{i}},
$$

then

$$
X_{f}=\frac{\partial f}{\partial p_{i}} \frac{\partial}{\partial q^{i}}-\frac{\partial f}{\partial q^{i}} \frac{\partial}{\partial p_{i}}
$$

and

$$
\{f, g\}=\frac{\partial f}{\partial p_{i}} \frac{\partial g}{\partial q^{i}}-\frac{\partial f}{\partial q^{i}} \frac{\partial g}{\partial p_{i}},
$$

the usual Poisson bracket of functions on phase-space. The corresponding bivector is $P=\frac{\partial}{\partial p_{i}} \wedge \frac{\partial}{\partial q^{i}}$.

In local coordinates, a necessary and sufficient condition for a bivector $P$ to be a Poisson bivector is

$$
\circlearrowleft P^{i \ell} \frac{\partial P^{j k}}{\partial x^{\ell}}=0
$$


where $\circlearrowleft$ denotes the sum over the circular permutations of $i, j, k$ (and the summation over $\ell=1,2, \ldots, \operatorname{dim} M$ is understood). In an invariant formalism, we define $\llbracket P, P \rrbracket$ to be the contravariant 3 -tensor with local components

$$
\llbracket P, P \rrbracket^{i j k}=-2 \circlearrowleft P^{i \ell} \frac{\partial P^{j k}}{\partial x^{\ell}} .
$$

One can show that this defines a tri-vector (skew-symmetric, contravariant 3tensor), called the Schouten bracket of $P$ (with itself), and that

$$
\llbracket P, P \rrbracket\left(d f_{1}, d f_{2}, d f_{3}\right)=-2 \circlearrowleft\left\{f_{1},\left\{f_{2}, f_{3}\right\}\right\} .
$$

From (3.5), it follows that $P$ is a Poisson bivector if and only if $\llbracket P, P \rrbracket=0$.

Alternatively, the bivector $P$ is a Poisson bivector if and only if

$$
X_{\{f, g\}}=\left[X_{f}, X_{g}\right], \text { for all } f, g \in C^{\infty}(M) .
$$

Now let $(M, \omega)$ be an arbitrary symplectic manifold, i.e., $\omega$ is a closed, nondegenerate differential 2-form on $M$. To say that $\omega$ is non-degenerate means that for each $x \in M, \underline{\omega}_{x}: T_{x} M \rightarrow T_{x}^{*} M$ defined by

$$
\left\langle\underline{\omega}_{x}\left(X_{x}\right), Y_{x}\right\rangle=\omega_{x}\left(Y_{x}, X_{x}\right),
$$

for $X_{x}, Y_{x} \in T_{x} M$, is a bijective linear map.

Let us show that every symplectic manifold has a Poisson structure. Since the linear mapping $\underline{\omega}_{x}$ is bijective, it has an inverse, which we denote by $\underline{P}_{x}$. As above, we set $X_{f}=\underline{P}(d f)=\underline{\omega}^{-1}(d f)$. Then

$$
\{f, g\}=X_{f} \cdot g=\left\langle\underline{\omega}\left(X_{g}\right), X_{f}\right\rangle=\omega\left(X_{f}, X_{g}\right) .
$$

First, one can show, using the classical properties of the Lie derivative, that $d \omega=0$ implies

$$
\left[X_{f}, X_{g}\right]=X_{\{f, g\}},
$$

for all $f$ and $g$ in $C^{\infty}(M)$. Now

$$
\circlearrowleft\left\{f_{1},\left\{f_{2}, f_{3}\right\}\right\}=X_{f_{1}} \cdot X_{f_{2}} \cdot f_{3}-X_{f_{2}} \cdot X_{f_{1}} \cdot f_{3}-X_{\left\{f_{1}, f_{2}\right\}} \cdot f_{3},
$$

which vanishes by the preceding observation. Thus, when $\omega$ is a closed, nondegenerate 2-form, the bracket $\{$,$\} satisfies the Jacobi identity. Therefore, if$ $\omega$ is a symplectic structure on $M$, then $\underline{P}$ defines a Poisson structure on $M$. Conversely, if $P$ is a Poisson bivector such that $\underline{P}$ is invertible, it defines a symplectic structure.

Whereas every symplectic manifold has a Poisson structure, the converse does not hold: a symplectic manifold is a Poisson manifold such that the linear map $\underline{P}_{x}$ is a bijection for each $x$ in $M$. 


\subsection{The dual of a Lie algebra}

We shall show that, given a finite-dimensional Lie algebra $\mathfrak{g}$, its dual $\mathfrak{g}^{*}$ is a Poisson manifold in a natural way. The Poisson structure on $\mathfrak{g}^{*}$ is defined by

$$
\underline{P}_{\xi}(x)=-a d_{x}^{*} \xi,
$$

where $\xi$ is a point of $\mathfrak{g}^{*}$, and $x \in \mathfrak{g}$ is considered as a 1 -form on $\mathfrak{g}^{*}$ at point $\xi$. This Poisson structure is called the linear Poisson structure (because $\underline{P}_{\xi}$ depends linearly on $\xi \in \mathfrak{g}^{*}$ ), or the Berezin-Kirillov-Kostant-Souriau Poisson structure (or a subset of those names), or the Lie-Poisson structure (not to be confused with the Poisson Lie structures on groups to be defined in Sect. 4.2).

For $f \in C^{\infty}\left(\mathfrak{g}^{*}\right), d_{\xi} f$ is in $T_{\xi}^{*} \mathfrak{g}^{*} \simeq\left(\mathfrak{g}^{*}\right)^{*} \simeq \mathfrak{g}$, and

$$
\underline{P}_{\xi}\left(d_{\xi} f\right)=-a d_{d_{\xi} f}^{*} \xi \in \mathfrak{g}^{*} \simeq T_{\xi} \mathfrak{g}^{*},
$$

so that

$$
X_{f}(\xi)=-a d_{d_{\xi} f}^{*} \xi
$$

while

$$
\{f, g\}(\xi)=X_{f}(\xi) \cdot g=-\left\langle a d_{d_{\xi} f}^{*} \xi, d_{\xi} g\right\rangle,
$$

whence

$$
\{f, g\}(\xi)=\left\langle\xi,\left[d_{\xi} f, d_{\xi} g\right]\right\rangle .
$$

If, in particular, $f=x, g=y$, where $x, y \in \mathfrak{g}$ are seen as linear forms on $\mathfrak{g}^{*}$, we obtain

$$
\{x, y\}(\xi)=\langle\xi,[x, y]\rangle
$$

It is clear that we have thus defined a Poisson structure on $\mathfrak{g}^{*}$ because, by (3.8), the Jacobi identity for $\{$,$\} follows from the Jacobi identity for the Lie bracket$ on $\mathfrak{g}$. In fact

$$
\circlearrowleft\left\{x_{1},\left\{x_{2}, x_{3}\right\}\right\}(\xi)=\left\langle\xi, \circlearrowleft\left[x_{1},\left[x_{2}, x_{3}\right]\right]\right\rangle=0 .
$$

From the Jacobi identity for linear functions on $\mathfrak{g}^{*}$, we deduce the Jacobi identity for polynomials, and hence for all smooth functions on $\mathfrak{g}^{*}$ (using a density theorem).

\subsection{The first Russian formula}

We now consider the case where $\mathfrak{g}$ is not only a Lie algebra, but has a Liebialgebra structure defined by an $r$-matrix, $r \in \mathfrak{g} \otimes \mathfrak{g}$. In this case $\mathfrak{g}^{*}$ is a Lie algebra, with Lie bracket $[,]^{r}$ such that

$$
\begin{aligned}
\left\langle[\xi, \eta]^{r}, x\right\rangle & =\delta r(x)(\xi, \eta)=\left(\left(a d_{x} \otimes 1+1 \otimes a d_{x}\right)(r)\right)(\xi, \eta) \\
& =[x \otimes 1+1 \otimes x, r](\xi, \eta),
\end{aligned}
$$


in the notations of Sect. 1.2, 2.1 and 2.3. Since the vector space $\mathfrak{g}$ can be identified with the dual of the Lie algebra $\mathfrak{g}^{*}$, it has the linear Poisson structure, here denoted by $\{,\}^{r}$,

$$
\{\xi, \eta\}^{r}(x)=\left\langle x,[\xi, \eta]^{r}\right\rangle
$$

whence

$$
\{\xi, \eta\}^{r}(x)=[x \otimes 1+1 \otimes x, r](\xi, \eta)
$$

Let us examine the case where $\mathfrak{g}$ is a Lie algebra of matrices. It is customary to write $L$ for a generic element in $\mathfrak{g}$ (because, in the theory of integrable systems, $L$ denotes the Lax matrix; see, e.g., Perelomov [25]). If $L$ is a $p \times p$ matrix, and 1 is the identity $p \times p$ matrix, then $L \otimes 1$ and $1 \otimes L$ are $p^{2} \times p^{2}$ matrices. If $L=\left(a_{i}^{j}\right)$, then

$$
L \otimes 1=\left(\begin{array}{ccccccccc}
a_{1}^{1} & 0 & \cdots & 0 & \cdots & a_{p}^{1} & 0 & \cdots & 0 \\
0 & a_{1}^{1} & \cdots & 0 & \cdots & 0 & a_{p}^{1} & \cdots & 0 \\
\vdots & \vdots & \ddots & \vdots & \ddots & \vdots & \vdots & \ddots & \vdots \\
0 & 0 & \cdots & a_{1}^{1} & \cdots & 0 & 0 & \cdots & a_{p}^{1} \\
\vdots & \vdots & \vdots & \vdots & \vdots & \vdots & \vdots & \vdots & \vdots \\
a_{1}^{p} & 0 & \cdots & 0 & \cdots & a_{p}^{p} & 0 & \cdots & 0 \\
0 & a_{1}^{p} & \cdots & 0 & \cdots & 0 & a_{p}^{p} & \cdots & 0 \\
\vdots & \vdots & \ddots & \vdots & \ddots & \vdots & \vdots & \ddots & \vdots \\
0 & 0 & \cdots & a_{1}^{p} & \cdots & 0 & 0 & \cdots & a_{p}^{p}
\end{array}\right), 1 \otimes L=\left(\begin{array}{cccc}
L & 0 & \cdots & 0 \\
0 & L & \cdots & 0 \\
& & \ddots & \\
0 & 0 & \cdots & L
\end{array}\right) .
$$

Since $r$ is an element in $\mathfrak{g} \otimes \mathfrak{g}$, it is also a $p^{2} \times p^{2}$ matrix. Then, what we have denoted by $[L \otimes 1+1 \otimes L, r]$ is the usual commutator of $p^{2} \times p^{2}$ matrices.

The Poisson structure of $\mathfrak{g} \simeq\left(\mathfrak{g}^{*}\right)^{*}$ is entirely specified if we know the Poisson brackets of the coordinate functions on $\mathfrak{g}$. Since $\mathfrak{g} \subset \mathfrak{g l}(p)$, it is enough to know the pairwise Poisson brackets of coefficients $\left\{a_{i}^{j}, a_{k}^{\ell}\right\}$, where $a_{i}^{j}$, for fixed indices $i, j \in\{1, \cdots, p\}$, is considered as the linear function on $\mathfrak{g}$ which associates its coefficient in the $i^{t h}$ column and $j^{\text {th }}$ row to a matrix $L \in \mathfrak{g}$. These Poisson brackets can be arranged in a $p^{2} \times p^{2}$ matrix, which we denote by $\{L \stackrel{\otimes}{,} L\}$. By definition, 


$$
\{L \stackrel{\otimes}{,} L\}=\left(\begin{array}{cccccc}
\left\{a_{1}^{1}, a_{1}^{1}\right\} & \cdots & \left\{a_{1}^{1}, a_{p}^{1}\right\} & \left\{a_{2}^{1}, a_{1}^{1}\right\} & \cdots & \left\{a_{p}^{1}, a_{p}^{1}\right\} \\
\left\{a_{1}^{1}, a_{1}^{2}\right\} & \cdots & \vdots & \vdots & & \vdots \\
\vdots & & \vdots & \vdots & & \vdots \\
\left\{a_{1}^{1}, a_{1}^{p}\right\} & \cdots & \left\{a_{1}^{1}, a_{p}^{p}\right\} & \cdots & \vdots \\
\left\{a_{1}^{2}, a_{1}^{1}\right\} & \cdots & \left\{a_{1}^{2}, a_{p}^{1}\right\} & \cdots & & \\
\vdots & & \vdots & & & \\
\left\{a_{1}^{2}, a_{1}^{p}\right\} & \cdots & \left\{a_{1}^{2}, a_{p}^{p}\right\} & \cdots & & \\
\vdots & & \vdots & & & \\
\left\{a_{1}^{p}, a_{1}^{p}\right\} & \cdots & \left\{a_{1}^{p}, a_{p}^{p}\right\} & \cdots & & \left\{a_{p}^{p}, a_{p}^{p}\right\}
\end{array}\right) .
$$

Now, evaluating the element $[L \otimes 1+1 \otimes L, r] \in \mathfrak{g} \otimes \mathfrak{g}$ on the pair $a_{i}^{j}, a_{k}^{\ell} \in \mathfrak{g}^{*}$, amounts to taking the $\begin{array}{lll}j & \ell \\ i & k\end{array}$ coefficient in the $p^{2} \times p^{2}$ matrix $[L \otimes 1+1 \otimes L, r]$. Thus formula (3.10) becomes the equality of matrices,

$$
\{L \stackrel{\otimes}{,} L\}=[L \otimes 1+1 \otimes L, r] .
$$

Sometimes the notations $L_{1}=L \otimes 1, L_{2}=1 \otimes L,\{L \stackrel{\otimes}{,} L\}=\left\{L_{1}, L_{2}\right\}$ are used. Then (3.11) becomes

$$
\left\{L_{1}, L_{2}\right\}=\left[L_{1}+L_{2}, r\right] .
$$

Formula (3.11) is what I call the first Russian formula. The second Russian formula to be explained in Sect. 4.3 will express the Poisson bracket of the coordinate functions on a Poisson Lie group when the elements of that group are matrices.

Remark. In formula (3.11), it is clear that $r$ can be assumed to be a skewsymmetric tensor, since its symmetric part is necessarily ad-invariant. This does not mean that the $p^{2} \times p^{2}$ matrix of $r$, nor that matrix $\left[L_{1}+L_{2}, r\right]$ is skew-symmetric.

Examples. We denote an element in $\mathfrak{s t}(2, \mathbb{C})$ by $L=\left(\begin{array}{ll}\alpha & \beta \\ \gamma & \delta\end{array}\right)$, with $\alpha+\delta=0$. Let $a=\frac{1}{4}(X \otimes Y-Y \otimes X) \in \bigwedge^{2}(\mathfrak{s l}(2, \mathbb{C}))$, as in Example 2 of Sect. 2.3. Then we obtain from formula (3.11),

$$
\{\alpha, \beta\}=\frac{1}{4} \beta,\{\alpha, \gamma\}=\frac{1}{4} \gamma,\{\beta, \gamma\}=0 .
$$


If we choose $r=X \otimes H-H \otimes X$, as in Example 3 of Sect. 2.3, then

$$
r=\left(\begin{array}{cccc}
0 & -1 & 1 & 0 \\
0 & 0 & 0 & -1 \\
0 & 0 & 0 & 1 \\
0 & 0 & 0 & 0
\end{array}\right) \text {, and }\{\alpha, \beta\}=-2 \alpha,\{\alpha, \gamma\}=0,\{\beta, \gamma\}=2 \gamma .
$$

\subsection{The traces of powers of Lax matrices are in involution}

We shall now show that whenever there exists an $r$-matrix $r$ such that (3.11) holds, the traces of powers of $L$ are pairwise in involution, i.e.,

$$
\left\{\operatorname{tr} L^{k}, \operatorname{tr} L^{\ell}\right\}=0
$$

for all $k, \ell \geq 1$. It will then follow that the eigenvalues of $L$ (as functions on $\mathfrak{g}$ ) are in involution.

For $p \times p$ matrices $A$ and $B$, we similarly define

$$
\{A \stackrel{\otimes}{,} B\}=\left\{A_{1}, B_{2}\right\} .
$$

First, we observe that

$$
\operatorname{tr}\left\{A_{1}, B_{2}\right\}=\{\operatorname{tr} A, \operatorname{tr} B\} .
$$

For matrices $A$ and $B$, it is clear that $(A B)_{1}=A_{1} B_{1}$ and $(A B)_{2}=A_{2} B_{2}$. It follows therefore from the Leibniz rule that, for matrices $A, B$ and $C$,

$$
\left\{A_{1},(B C)_{2}\right\}=\left\{A_{1}, B_{2} C_{2}\right\}=B_{2}\left\{A_{1}, C_{2}\right\}+\left\{A_{1}, B_{2}\right\} C_{2} .
$$

Induction then shows that for any matrix $L$,

$$
\left\{L_{1}^{k}, L_{2}^{\ell}\right\}=\sum_{a=0}^{k-1} \sum_{b=0}^{\ell-1} L_{1}^{k-a-1} L_{2}^{\ell-b-1}\left\{L_{1}, L_{2}\right\} L_{1}^{a} L_{2}^{b} .
$$

From this relation, we obtain

$$
\left\{\operatorname{tr} L^{k}, \operatorname{tr} L^{\ell}\right\}=\operatorname{tr}\left\{L_{1}^{k}, L_{2}^{\ell}\right\}=k \ell \operatorname{tr} L_{1}^{k-1} L_{2}^{\ell-1}\left\{L_{1}, L_{2}\right\},
$$

since $A_{1} B_{2}=(A \otimes 1)(1 \otimes B)=A \otimes B=(1 \otimes B)(A \otimes 1)=B_{2} A_{1}$ for any matrices $A$ and $B$. Now, we use $\left(3.11^{\prime}\right)$ and we obtain

$$
\left\{\operatorname{tr} L^{k}, \operatorname{tr} L^{\ell}\right\}=k \ell \operatorname{tr} L_{1}^{k-1} L_{2}^{\ell-1}\left[L_{1}+L_{2}, r\right] .
$$

Now $\operatorname{tr}\left(L_{1}^{k-1} L_{2}^{\ell-1} L_{1} r-L_{1}^{k-1} L_{2}^{\ell-1} r L_{1}\right)=0$, and similarly $\operatorname{tr}\left(L_{1}^{k-1} L_{2}^{\ell-1} L_{2} r-\right.$ $\left.L_{1}^{k-1} L_{2}^{\ell-1} r L_{2}\right)=0$, and relation (3.12) is proved. (This result can be obtained as a corollary of Semenov-Tian-Shansky's theorem on double Lie algebras to be stated in Sect. 3.6.) 
We know that when a dynamical system is in Lax form, i.e., can be written as

$$
\dot{L}=[L, B]
$$

where $L$ and $B$ are matrices depending on the phase-space coordinates, the eigenvalues of $L$ are conserved (isospectral evolution), and hence the traces of powers of $L$ are conserved. What we have proved is the following

Proposition. When the Poisson brackets of the coefficients of the Lax matrix are given by an r-matrix, the traces of powers of the Lax matrix are conserved quantities in involution.

If sufficiently many of these conserved quantites in involution are functionally independent, the complete integrability of the system in the sense of Liouville and Arnold (see Arnold [2]) follows.

Remark. Relation (3.12) is valid under the weaker assumption that $r$ is an $r$-matrix in the sense of Remark 2 of Sect. 2.1. In this more general case, we obtain from $[\xi, \eta]^{r}=a d_{\underline{r} \xi}^{*} \eta-a d_{\underline{r} \eta}^{*} \xi$,

$$
\left\{L_{1}, L_{2}\right\}=\left[L_{1},-{ }^{t} r\right]+\left[L_{2}, r\right]=\left[{ }^{t} r, L_{1}\right]-\left[r, L_{2}\right] .
$$

(This is formula (11) of Babelon and Viallet [35], where $d={ }^{t} r$.)

\subsection{Symplectic leaves and coadjoint orbits}

We have remarked in Sect. 3.1 that any symplectic manifold is a Poisson manifold but that the converse does not hold. In fact, any Poisson manifold is a union of symplectic manifolds, generally of varying dimensions, called the leaves of the symplectic foliation of the Poisson manifold. (In the case of a symplectic manifold, there is only one such leaf, the manifold itself.) On a Poisson manifold $(M, P)$, let us consider an open set where the rank of $\underline{P}$ is constant. At each point $x$, the image of $T_{x}^{*} M$ under $\underline{P}_{x}$ is a linear subspace of $T_{x} M$ of dimension equal to the rank of $\underline{P}_{x}$. That the distribution $x \mapsto \operatorname{Im} \underline{P}_{x}$ is integrable follows from the Poisson property of $P$. (Here, 'distribution' means a vector subbundle of the tangent bundle. See, e.g., Vaisman [30].) By the Frobenius theorem, this distribution defines a foliation, whose leaves are the maximal integral manifolds. If the rank of $\underline{P}_{x}$ is not constant on the manifold, this distribution defines a generalized foliation, the leaves of which are of varying dimension equal to the rank of the Poisson map. This will be illustrated in the case of the dual of a Lie algebra.

First, let us review the adjoint and coadjoint actions of Lie groups.

Let $G$ be a Lie group with Lie algebra $\mathfrak{g}$. Then $G$ acts on its Lie algebra by the adjoint action, denoted by $A d$. For $g \in G, x \in \mathfrak{g}$,

$$
A d_{g} x=\left.\frac{d}{d t}\left(g \cdot \exp t x \cdot g^{-1}\right)\right|_{t=0} .
$$


If $G$ is a matrix Lie group, then $g$ and $x$ are matrices and we recover the usual formula,

$$
A d_{g} x=g x g^{-1} .
$$

By definition, the adjoint orbit of $x \in \mathfrak{g}$ is the set of all $A d_{g} x$, for $g \in G$. In the case of a matrix Lie group, it is the set of matrices which are conjugate to $x$ by matrices belonging to $G$.

If we define

$$
a d_{x} y=\left.\frac{d}{d t}\left(A d_{\exp t x} y\right)\right|_{t=0}
$$

then

$$
a d_{x} y=\left.\frac{d}{d t} \frac{d}{d s} \exp t x \cdot \exp s y \cdot \exp (-t x)\right|_{t=s=0}=[x, y] .
$$

Thus ad does coincide with the adjoint representation of $\mathfrak{g}$ on itself introduced in Sect. 1.2.

We can define an action of $G$ on functions on $\mathfrak{g}$ by

$$
(g . f)(x)=f\left(A d_{g^{-1}} x\right), \text { for } x \in \mathfrak{g}, f \in C^{\infty}(\mathfrak{g}), g \in G \text {. }
$$

A function $f$ on $\mathfrak{g}$ is called Ad-invariant (or G-invariant) if $g . f=f$, for all $g \in G$. This means that $f$ is constant on the orbits of the adjoint action. In the case of a group of matrices, it means that the value of $f$ at $x$ depends only on the equivalence class of $x$, modulo conjugation by elements of $G$. For example, we can take $f(x)=\frac{1}{k} \operatorname{tr}\left(x^{k}\right)$, for $k$ a positive integer.

We now consider the dual $\mathfrak{g}^{*}$ of $\mathfrak{g}$, and we define the coadjoint action of $G$ on $\mathfrak{g}^{*}$, denoted by $A d^{*}$, as

$$
\left\langle A d_{g}^{*} \xi, x\right\rangle=\left\langle\xi, A d_{g^{-1}} x\right\rangle
$$

for $g \in G, \xi \in \mathfrak{g}^{*}, x \in \mathfrak{g}$. By definition, the coadjoint orbit of $\xi \in \mathfrak{g}^{*}$ is the set of all $A d_{g}^{*} \xi$, for $g \in G$. If we define

$$
a d_{x}^{*} \xi=\left.\frac{d}{d t} A d_{\exp t x}^{*} \xi\right|_{t=0}
$$

we see that $a d^{*}$ coincides with the coadjoint representation of $\mathfrak{g}$ on $\mathfrak{g}^{*}$ which we introduced in Sect. 1.3. The tangent space at $\xi \in \mathfrak{g}^{*}$ to the coadjoint orbit $\mathcal{O}_{\xi}$ of $\xi$ is the linear subspace of $T_{\xi} \mathfrak{g}^{*} \simeq \mathfrak{g}^{*}$,

$$
T_{\xi} \mathcal{O}_{\xi}=\left\{a d_{x}^{*} \xi \mid x \in \mathfrak{g}\right\}
$$

The Lie group $G$ acts on functions on $\mathfrak{g}^{*}$ by

$$
(g . f)(\xi)=f\left(A d_{g^{-1}}^{*} \xi\right), \text { for } \xi \in \mathfrak{g}^{*}, f \in C^{\infty}\left(\mathfrak{g}^{*}\right), g \in G .
$$


A function $f$ on $\mathfrak{g}^{*}$ is called $A d^{*}$-invariant (or $G$-invariant) if $g . f=f$, for all $g \in G$.

Now we give infinitesimal characterizations of $A d$-invariant functions on $\mathfrak{g}$ and $A d^{*}$-invariant functions on $\mathfrak{g}^{*}$.

Proposition. Assume that the Lie group $G$ is connected. A function $f \in C^{\infty}(\mathfrak{g})$ is Ad-invariant if and only if

$$
a d_{x}^{*}\left(d_{x} f\right)=0, \text { for all } x \in \mathfrak{g} .
$$

A function $f \in C^{\infty}\left(\mathfrak{g}^{*}\right)$ is $A d^{*}$-invariant if and only if

$$
a d_{d_{\xi} f}^{*} \xi=0, \text { for all } \xi \in \mathfrak{g}^{*} .
$$

Proof. Let $f$ be a function on $\mathfrak{g}$. If $G$ is connected, by (3.16), relation $g . f=f$ is satisfied for all $g \in G$ if and only if

$$
0=\left.\frac{d}{d t} f\left(A d_{\exp (-t y)} x\right)\right|_{t=0}=-\left\langle d_{x} f,[y, x]\right\rangle=-\left\langle a d_{x}^{*}\left(d_{x} f\right), y\right\rangle,
$$

for all $x, y \in \mathfrak{g}$, proving (3.20). Similarly, if $f \in C^{\infty}\left(\mathfrak{g}^{*}\right)$, then by (3.19), relation $g . f=f$ is satisfied for all $g \in G$ if and only if

$$
0=\left.\frac{d}{d t} f\left(A d_{\exp (-t x)}^{*} \xi\right)\right|_{t=0}=-\left\langle d_{\xi} f, a d_{x}^{*} \xi\right\rangle=\left\langle x, a d_{d_{\xi} f}^{*} \xi\right\rangle
$$

for all $x \in \mathfrak{g}, \xi \in \mathfrak{g}^{*}$, proving (3.21).

Note that alternate ways of writing (3.20) and (3.21) are

$$
d_{x} f \circ a d_{x}=0 \text {, for all } x \in \mathfrak{g}
$$

and

$$
\xi \circ a d_{d_{\xi} f}=0 \text {, for all } \xi \in \mathfrak{g}^{*} \text {. }
$$

For example, if $f(x)=\frac{1}{k} \operatorname{tr}\left(x^{k}\right)$, then $d_{x} f(h)=\operatorname{tr}\left(x^{k-1} h\right)$, for $x, h \in \mathfrak{g}$, and it is clear that, for each $y \in \mathfrak{g}$,

$$
\operatorname{tr}\left(x^{k-1}[x, y]\right)=0 .
$$

Let us examine the case where $\mathfrak{g}^{*}$ can be identified with $\mathfrak{g}$ by means of an $a d$-invariant, non-degenerate, symmetric, bilinear form $s$. This is the case if $\mathfrak{g}$ is semi-simple and we choose $s$ to be the Killing form. Then $\underline{s}: \mathfrak{g}^{*} \rightarrow \mathfrak{g}$ satisfies

$$
a d_{x} \circ \underline{s}=\underline{s} \circ a d_{x}^{*},
$$


for all $x \in \mathfrak{g}$ (see Sect. 2.1), so that in this identification the coadjoint action becomes the adjoint action. If $f$ is a function on $\mathfrak{g}^{*} \simeq \mathfrak{g}$, its differential at a point $L$ is an element in $\mathfrak{g}$. By (3.20), the function $f$ is $G$-invariant if and only if

$$
\left[L, d_{L} f\right]=0
$$

We can now prove

Proposition. Let $G$ be a Lie group with Lie algebra $\mathfrak{g}$. The symplectic leaves of the linear Poisson structure of $\mathfrak{g}^{*}$ are the connected components of the coadjoint orbits in $\mathfrak{g}^{*}$.

Proof. By (3.7), the linear Poisson bivector $P$ on $\mathfrak{g}^{*}$ is such that

$$
\operatorname{Im} \underline{P}_{\xi}=\left\{a d_{x}^{*} \xi \mid x \in \mathfrak{g}\right\}
$$

Thus, by (3.18), the image of $\underline{P}_{\xi}$ coincides with the tangent space at $\xi \in \mathfrak{g}^{*}$ to the coadjoint orbit of $\xi$. The result follows.

Example. If $G=S O(3), \mathfrak{g}^{*}$ is a 3 -dimensional vector space identified with $\mathbb{R}^{3}$ in which the coadjoint orbits are the point $\{0\}$ (0-dimensional orbit) and all spheres centered at the origin (2-dimensional orbits).

Note that the coadjoint orbit of the origin 0 in the dual of any Lie algebra is always $\{0\}$. In particular, the dual of a Lie algebra is not a symplectic manifold.

When a Poisson manifold is not symplectic, there are nonconstant functions which are in involution with all functions on the manifold.

Definition. In a Poisson manifold, those functions whose Poisson brackets with all functions vanish are called Casimir functions.

Proposition. In the dual of the Lie algebra of a connected Lie group $G$, the Casimir functions are the $A d^{*}$-invariant functions.

Proof. In fact, if $f, g \in C^{\infty}\left(\mathfrak{g}^{*}\right)$ and $\xi \in \mathfrak{g}^{*}$, then

$$
\{f, g\}(\xi)=\left\langle\xi,\left[d_{\xi} f, d_{\xi} g\right]\right\rangle=-\left\langle a d_{d_{\xi} f}^{*} \xi, d_{\xi} g\right\rangle .
$$

This quantity vanishes for all $g \in C^{\infty}\left(\mathfrak{g}^{*}\right)$ if and only if $a d_{d_{\xi} f}^{*} \xi=0$, and therefore this proposition is a consequence of $(3.21)$.

The symplectic leaves of a Poisson manifold are contained in the connected components of the level sets of the Casimir functions. (On a symplectic leaf, each Casimir function is constant.) 


\subsection{Double Lie algebras and Lax equations}

Let $(\mathfrak{g}, R)$ be a double Lie algebra in the sense of Sect. 2.4. Then $\mathfrak{g}$ has two Lie algebra structures, $[$,$] and [,]_{R}$ defined by (2.11). We denote the corresponding adjoint (resp., coadjoint) actions by $a d$ and $a d^{R}$ (resp., $a d^{*}$ and $a d^{R *}$ ). Thus $\mathfrak{g}^{*}$ has two linear Poisson structures, $P$ and $P^{R}$. By definition, for $x, y \in \mathfrak{g}, \xi \in \mathfrak{g}^{*}$,

$$
\begin{gathered}
\left\langle\underline{P}_{\xi}^{R}(x), y\right\rangle=-\left\langle a d_{x}^{R *} \xi, y\right\rangle=\left\langle\xi,[x, y]_{R}\right\rangle \\
=\langle\xi,[R x, y]+[x, R y]\rangle,
\end{gathered}
$$

whence

$$
\left\langle\underline{P}_{\xi}^{R}(x), y\right\rangle=-\left\langle a d_{R x}^{*} \xi, y\right\rangle-\left\langle a d_{x}^{*} \xi, R y\right\rangle
$$

We denote the Poisson bracket defined by $P^{R}$ by $\{,\}_{R}$. For $f_{1}, f_{2} \in C^{\infty}\left(\mathfrak{g}^{*}\right)$,

$$
\begin{gathered}
\left\{f_{1}, f_{2}\right\}_{R}(\xi)=-\left\langle a d_{R\left(d_{\xi} f_{1}\right)}^{*} \xi, d_{\xi} f_{2}\right\rangle-\left\langle a d_{d_{\xi} f_{1}}^{*} \xi, R\left(d_{\xi} f_{2}\right)\right\rangle \\
=\left\langle a d_{d_{\xi} f_{2}}^{*} \xi, R\left(d_{\xi} f_{1}\right)\right\rangle-\left\langle a d_{d_{\xi} f_{1}}^{*} \xi, R\left(d_{\xi} f_{2}\right)\right\rangle
\end{gathered}
$$

From this formula and from the proof of the last proposition of Sect. 3.5, we obtain

Theorem. Let $\mathfrak{g}^{*}$ be the dual of a double Lie algebra, with Poisson brackets $\{$,$\} and \{,\}_{R}$. If $f_{1}$ and $f_{2}$ are Casimir functions on $\left(\mathfrak{g}^{*},\{\},\right)$, they are in involution with respect to $\{,\}_{R}$.

Now, let $f$ be a Casimir function on $\left(\mathfrak{g}^{*},\{\},\right)$. Its Hamiltonian vector field $X_{f}$ clearly vanishes. Let us denote the Hamiltonian vector field with Hamiltonian $f$ with respect to $\{,\}_{R}$ by $X_{f}^{R}$. Then

$$
X_{f}^{R}(\xi)=\underline{P}_{\xi}^{R}\left(d_{\xi} f\right) .
$$

If $f$ is a Casimir function on $\left(\mathfrak{g}^{*},\{\},\right)$, it follows from (3.22) and (3.21) that

$$
X_{f}^{R}(\xi)=-a d_{R\left(d_{\xi} f\right)}^{*} \xi .
$$

The corresponding Hamiltonian equation is $\dot{\xi}=-a d_{R\left(d_{\xi} f\right)}^{*} \xi$. In addition, let us assume that $\mathfrak{g}^{*}$ is identified with $\mathfrak{g}$ by an $a d$-invariant, nondegenerate, symmetric $s \in \mathfrak{g} \otimes \mathfrak{g}$. Then, denoting a generic element in $\mathfrak{g}$ by $L$, the element $-a d_{R\left(d_{\xi} f\right)}^{*} \xi$ in $\mathfrak{g}^{*}$ is identified with $-a d_{R\left(d_{L} f\right)} L=\left[L, R\left(d_{L} f\right)\right]$ in $\mathfrak{g}$. Thus the Hamiltonian vector field satisfies

$$
X_{f}^{R}(L)=\left[L, R\left(d_{L} f\right)\right],
$$

and the corresponding Hamiltonian equation is

$$
\dot{L}=\left[L, R\left(d_{L} f\right)\right]
$$


which is in fact an equation of Lax type, $\dot{L}=[L, B]$.

The theorem and these formulæ constitute Semenov-Tian-Shansky's theorem.

If, in particular, $\mathfrak{g}=\mathfrak{g}_{+} \oplus \mathfrak{g}_{-}$, where $\mathfrak{g}_{+}$and $\mathfrak{g}_{-}$are Lie subalgebras, and $R=\frac{1}{2}\left(p_{+}-p_{-}\right)$, where $p_{+}$(resp., $\left.p_{-}\right)$is the projection onto $\mathfrak{g}_{+}$(resp., $\mathfrak{g}_{-}$) parallel to $\mathfrak{g}_{-}$(resp., $\mathfrak{g}_{+}$) (see the example in Sect. 2.4), then for a Casimir function $f$ on $\left(\mathfrak{g}^{*},\{\},\right)$, from (3.23) and (3.21), we obtain

$$
X_{f}^{R}(\xi)=-\frac{1}{2} a d_{\left(d_{\xi} f\right)_{+}-\left(d_{\xi} f\right)_{-}}^{*} \xi=-a d_{\left(d_{\xi} f\right)_{+}}^{*} \xi=a d_{\left(d_{\xi} f\right)_{-}}^{*} \xi,
$$

where $x_{ \pm}=p_{ \pm}(x)$, or, in the Lax form,

$$
\dot{L}=\left[L,\left(d_{L} f\right)_{+}\right],
$$

or, equivalently,

$$
\dot{L}=-\left[L,\left(d_{L} f\right)_{-}\right] .
$$

Example. Let $\mathfrak{g}=\mathfrak{s l}(n, \mathbb{R})$, and let $\mathfrak{g}_{+}=\mathfrak{s} \mathfrak{o}(n), \mathfrak{g}_{-}$the subalgebra of upper triangular matrices (see the example of Sect 2.4), where $\mathfrak{g}^{*}$ and $\mathfrak{g}$ are identified by the trace functional $\operatorname{tr}(x y)$, and let us choose $f(L)=\frac{1}{2} \operatorname{tr} L^{2}$.

Then (3.25) becomes

$$
\dot{L}=\left[L, L_{+}\right],
$$

where $L=L_{+}+L_{-}$and $L_{ \pm} \in \mathfrak{g}_{ \pm}$.

The various results of this section are of importance in the theory of integrable systems because they furnish conserved quantities in involution. If $h$ is a Casimir function of $(\mathfrak{g},[]$,$) , the Hamiltonian vector field X_{h}^{R}$ is tangent to the coadjoint orbits of $\left(\mathfrak{g},[,]_{R}\right)$. In restriction to an orbit, which is a symplectic manifold, we obtain a Hamiltonian system, for which the restrictions of the Casimir functions of $\left(\mathfrak{g}^{*},\{\},\right)$ are conserved quantities in involution.

For example, by restricting the Hamiltonian system of the preceding example to the adjoint orbit of the matrix

$$
\left(\begin{array}{ccccc}
0 & 1 & 0 & \cdots & 0 \\
1 & 0 & 0 & \cdots & 0 \\
\vdots & \vdots & \vdots & \vdots & \vdots \\
0 & 0 & \cdots & 0 & 1 \\
0 & 0 & \cdots & 1 & 0
\end{array}\right)
$$

in $\mathfrak{s l}(n, \mathbb{R})$, we obtain the Toda system, for which $\frac{1}{k} \operatorname{tr}\left(L^{k}\right)$ are conserved quantities in involution. 
Let us show that Semenov-Tian-Shansky's theorem implies the Adler-KostantSymes theorem.

Let us denote the orthogonal of $\mathfrak{g}_{+}$(resp., $\left.\mathfrak{g}_{-}\right)$in $\mathfrak{g}^{*}$ by $\mathfrak{g}_{+}^{\perp}$ (resp., $\left.\mathfrak{g}_{-}^{\perp}\right)$. Then $\mathfrak{g}_{+}^{*}$ can be identified with $\mathfrak{g}_{-}^{\perp}$, while $\mathfrak{g}_{-}^{*}$ can be identified with $\mathfrak{g}_{+}^{\perp}$, and $\mathfrak{g}^{*}$ splits as

$$
\mathfrak{g}^{*}=\mathfrak{g}_{-}^{\perp} \oplus \mathfrak{g}_{+}^{\perp} \approx \mathfrak{g}_{+}^{*} \oplus \mathfrak{g}_{-}^{*} .
$$

If $f$ is a function on $\mathfrak{g}^{*}$, then

$$
d_{\xi}\left(\left.f\right|_{\mathfrak{g}_{+}^{*}}\right)=\left(d_{\xi} f\right)_{+} .
$$

It follows that, for functions $f_{1}$ and $f_{2}$ on $\mathfrak{g}^{*}$,

$$
\left\{\left.f_{1}\right|_{\mathfrak{g}_{+}^{*}},\left.f_{2}\right|_{\mathfrak{g}_{+}^{*}}\right\}_{\mathfrak{g}_{+}^{*}}=\left.\left\{f_{1}, f_{2}\right\}_{R}\right|_{\mathfrak{g}_{+}^{*}}
$$

where $\{,\}_{\mathfrak{g}_{+}^{*}}$ denotes the Lie-Poisson bracket on the dual of the Lie algebra $\mathfrak{g}_{+}$. In fact, by formula (2.19), both sides evaluated at $\xi \in \mathfrak{g}_{+}^{*}$ are equal to $\left\langle\xi,\left[\left(d_{\xi} f_{1}\right)_{+},\left(d_{\xi} f_{2}\right)_{+}\right]\right\rangle$. By the preceding theorem, if $f_{1}$ and $f_{2}$ are Casimir functions on $\mathfrak{g}^{*}$, they satisfy $\left\{f_{1}, f_{2}\right\}_{R}=0$, and therefore, by (3.28),

$$
\left\{\left.f_{1}\right|_{\mathfrak{g}_{+}^{*}},\left.f_{2}\right|_{\mathfrak{g}_{+}^{*}}\right\}_{\mathfrak{g}_{+}^{*}}=0 .
$$

Thus we obtain the Adler-Kostant-Symes theorem, namely,

Theorem. Let $\mathfrak{g}=\mathfrak{g}_{+} \oplus \mathfrak{g}_{-}$, where $\mathfrak{g}_{+}$and $\mathfrak{g}_{-}$are Lie subalgebras of $\mathfrak{g}$, and let $f_{1}$ and $f_{2}$ be Casimir functions on $\mathfrak{g}^{*}$. Then the restrictions of $f_{1}$ and $f_{2}$ to $\mathfrak{g}_{-}^{\perp} \approx \mathfrak{g}_{+}^{*}$ commute in the Lie-Poisson bracket of $\mathfrak{g}_{+}^{*}$.

\subsection{Solution by factorization}

We shall show that, when the $R$-matrix is defined by the decomposition of a Lie algebra into a sum of complementary Lie subalgebras, the problem of integrating dynamical system (3.23) can be reduced to a factorization problem in the associated Lie group. Thus in this case, the Lax equation (3.24) can be solved "by factorization". Actually this scheme is valid in the more general situation where $R$ is a factorizable $R$-matrix, and this fact explains the terminology.

Let $G$ be a Lie group with Lie algebra $\mathfrak{g}=\mathfrak{g}_{+} \oplus \mathfrak{g}_{-}$, where $\mathfrak{g}_{+}$and $\mathfrak{g}_{-}$are Lie subalgebras of $\mathfrak{g}$, and let $G_{+}$(resp., $G_{-}$) be the connected Lie subgroup of $G$ with Lie algebra $\mathfrak{g}_{+}$(resp., $\left.\mathfrak{g}_{-}\right)$. The solution by factorization of the initial value problem,

$$
\left\{\begin{array}{l}
\dot{\xi}=-a d_{\left(d_{\xi} f\right)_{+}}^{*} \xi \\
\xi(0)=\xi_{0}
\end{array}\right.
$$

where $f$ is a Casimir function on $\mathfrak{g}^{*}$, and $\xi_{0}$ is an element of $\mathfrak{g}^{*}$, is the following.

Let $x_{0}=d_{\xi_{0}} f \in \mathfrak{g}$, and assume that $e^{t x_{0}} \in G$ has been factorized as

$$
e^{t x_{0}}=g_{+}(t)^{-1} g_{-}(t)
$$


where $g_{ \pm}(t) \in G_{ \pm}, g_{ \pm}(0)=e$, which is possible for $|t|$ small enough. We shall prove that

$$
\xi(t)=A d_{g_{+}(t)}^{*} \xi_{0}
$$

solves the preceding initial-value problem.

In fact, because $f$ is $A d^{*}$-invariant, for $g \in G, \eta \in \mathfrak{g}^{*}$,

$$
\begin{gathered}
\left(d_{A d_{g}^{*} \xi_{0}} f\right)(\eta)=\left.\frac{d}{d s} f\left(A d_{g}^{*} \xi_{0}+s \eta\right)\right|_{s=0} \\
=\left.\frac{d}{d s} f\left(\xi_{0}+s A d_{g^{-1}}^{*} \eta\right)\right|_{s=0}=\left(d_{\xi_{0}} f\right)\left(A d_{g^{-1}}^{*} \eta\right),
\end{gathered}
$$

so that

$$
d_{A d_{g}^{*} \xi_{0}} f=A d_{g}\left(d_{\xi_{0}} f\right) .
$$

Now, from $g_{+}(t) e^{t x_{0}}=g_{-}(t)$, we obtain, by differentiating,

$$
\dot{g}_{+}(t) g_{+}(t)^{-1}+A d_{g_{+}(t)} x_{0}=\dot{g}_{-}(t) g_{-}(t)^{-1} .
$$

Using the definition of $x_{0}$, and formulæ (3.30) (3.31), we obtain

$$
d_{\xi(t)} f=-\dot{g}_{+}(t) g_{+}(t)^{-1}+\dot{g}_{-}(t) g_{-}(t)^{-1},
$$

so that

$$
\left(d_{\xi(t)} f\right)_{+}=-\dot{g}_{+}(t) g_{+}(t)^{-1},
$$

while it follows from (3.30) that

$$
\dot{\xi}(t)=a d_{\dot{g}_{+}(t) g_{+}(t)^{-1}}^{*} \xi(t) .
$$

Therefore $\xi(t)$ given by (3.30) solves the given initial-value problem.

\subsection{Bibliographical note}

For Poisson manifolds and coadjoint orbits, see Cartier [21], Vaisman [30] or Marsden and Ratiu [12]. For Sect. 3.3, see Faddeev and Takhtajan [23], Babelon and Viallet [20]. Double Lie algebras were introduced by Semenov-Tian-Shansky [18] [28], where the theorem of Sect. 3.6 is proved, and the factorization method is explained. For the Toda system, see Kostant [9], Reyman and SemenovTian-Shansky [27], Semenov-Tian-Shansky [28], Babelon and Viallet [20]. For the Adler-Kostant-Symes theorem, see Kostant [9], Guillemin and Sternberg [7]. The books by Faddeev and Takhtajan [23] and by Perelomov [25] contain surveys of the Lie-algebraic approach to integrable equations. 


\section{Poisson Lie groups}

When a Lie group is also a Poisson manifold, it is natural to require that the Poisson structure and the multiplication defining the group structure be compatible in some sense. This idea, when made precise, leads to the notion of a Poisson Lie group. In fact, the introduction of Poisson Lie groups was motivated by the properties of the monodromy matrices of difference equations describing lattice integrable systems. We shall give the definition of Poisson Lie groups and show that the infinitesimal object corresponding to a Poisson Lie group is a Lie bialgebra. The emphasis will be on Poisson Lie groups defined by $r$-matrices, and we shall study examples on matrix groups.

It has gradually emerged from the physics literature that the group of dressing transformations should be considered as the action of a Poisson Lie group on a Poisson manifold. This action is a Poisson action in a sense that we shall define in Sect. 4.7, and which generalizes the Hamiltonian actions. For such actions one can define a momentum mapping (also known as a non-Abelian Hamiltonian). If $G$ and $G^{*}$ are dual Poisson Lie groups, the dressing action of $G^{*}$ on $G$, and that of $G^{*}$ on $G$ will be defined and characterized in various ways in Sect. 4.9 .

\subsection{Multiplicative tensor fields on Lie groups}

First recall that in a Lie group $G$, the left- and right-translations by an element $g \in G$, denoted by $\lambda_{g}$ and $\rho_{g}$ respectively, are defined by

$$
\lambda_{g}(h)=g h, \rho_{g}(h)=h g,
$$

for $h \in G$. Taking the tangent linear map to $\lambda_{g}$ (resp., $\rho_{g}$ ) at point $h \in G$, we obtain a linear map from $T_{h} G$, the tangent space to $G$ at $h$, into the tangent space to $G$ at $\lambda_{g}(h)=g h$ (resp., at $\rho_{g}(h)=h g$ ). For any positive integer $k$, using the $k$-th tensor power of the tangent map to $\lambda_{g}$ (resp., $\rho_{g}$ ), we can map $k$-tensors at $h$ to $k$-tensors at $g h$ (resp., $h g$ ). If $Q_{h}$ is a tensor at $h$, we simply denote its image under this map by $g . Q_{h}$ (resp., $Q_{h} . g$ ). Here and below, we write tensor for smooth contravariant tensor field.

Definition. A tensor $Q$ on a Lie group $G$ is called multiplicative if

$$
Q_{g h}=g \cdot Q_{h}+Q_{g} \cdot h
$$

for all $g, h \in G$.

Note that this relation implies that

$$
Q_{e}=0
$$

where $e$ is the unit element of the group $G$. In fact, setting $h=g=e$ in (4.1), we obtain $Q_{e}=2 Q_{e}$. 
Let us denote the Lie algebra of $G$, which is the tangent space $T_{e} G$ to $G$ at $e$, by $\mathfrak{g}$. We can associate to any $k$-tensor $Q$ on the Lie group $G$ a mapping $\rho(Q)$ from $G$ to the $k$-th tensor power of $\mathfrak{g}$ defined by

$$
\rho(Q)(g)=Q_{g} \cdot g^{-1},
$$

for $g \in G$. When $Q$ is multiplicative, the mapping $\rho(Q)$ has the following property. For all $g, h \in G$,

$$
\rho(Q)(g h)=A d_{g}(\rho(Q)(h))+\rho(Q)(g) .
$$

In fact, by (4.3) and (4.1),

$$
\begin{aligned}
\rho(Q)(g h) & =Q_{g h} \cdot(g h)^{-1}=\left(g \cdot Q_{h}+Q_{g} \cdot h\right) \cdot h^{-1} \cdot g^{-1} \\
& =g \cdot \rho(Q)(h) \cdot g^{-1}+\rho(Q)(g)
\end{aligned}
$$

Taking into account the definition of the adjoint action of the group on tensor powers of $\mathfrak{g}, A d_{g} a=$ g.a.g $g^{-1}$, for $a \in \otimes^{k} \mathfrak{g}$, we obtain (4.4). Observe that if $a=a_{1} \otimes \cdots \otimes a_{k}$, then $g \cdot a=g \cdot a_{1} \otimes \cdots \otimes g \cdot a_{k}$.

Definition. A mapping $U$ from $G$ to a representation space $(V, \mathcal{R})$ of $G$ satisfying

$$
U(g h)=\mathcal{R}(g)(U(h))+U(g)
$$

is called a 1-cocycle of $G$ with values in $V$, with respect to the representation $\mathcal{R}$.

Thus we have proved

Proposition. If $Q$ is a multiplicative $k$-tensor on a Lie group $G$, then $\rho(Q)$ is a 1-cocycle of $G$ with values in the $k$-th tensor power of $\mathfrak{g}$, with respect to the adjoint action of $G$.

As the names suggest, there is a relationship between Lie-group cocycles and Lie-algebra cocycles. The following is a well-known result.

Proposition. If $U: G \rightarrow V$ is a 1-cocycle of $G$ with values in $V$, with respect to the representation $\mathcal{R}$, then $T_{e} U: \mathfrak{g} \rightarrow V$ defined by

$$
T_{e} U(x)=\left.\frac{d}{d t} U(\exp t x)\right|_{t=0},
$$

for $x \in \mathfrak{g}$, is a 1 -cocycle of $\mathfrak{g}$ with values in $V$, with respect to the representation $d_{e} \mathcal{R}$ defined by

$$
d_{e} \mathcal{R}(x)=\left.\frac{d}{d t} \mathcal{R}(\exp t x)\right|_{t=0} .
$$

Conversely, if $u$ is a 1-cocyle of $\mathfrak{g}$ with values in $V$, with respect to a representation $\sigma$ of $\mathfrak{g}$, and if $G$ is connected and simply connected, there exists a unique 
1-cocyle $U$ of $G$ with values in $V$, with respect to the representation $\mathcal{R}$ such that $d_{e} \mathcal{R}=\sigma$, satisfying $T_{e} U=u$.

To any multiplicative $k$-tensor $Q$, we associate the 1 -cocycle on $\mathfrak{g}$ with respect to the adjoint action of $\mathfrak{g}$ on the $k$-th tensor power of $\mathfrak{g}$,

$$
D Q=T_{e}(\rho(Q)) .
$$

This linear map from $\mathfrak{g}$ to $\stackrel{k}{\otimes} \mathfrak{g}$ coincides with the linearization of $Q$ at $e$, namely it satisfies, for $x \in \mathfrak{g}$,

$$
(D Q)(x)=\left(\mathcal{L}_{X} Q\right)(e),
$$

where $X$ is any vector field on $G$ defined in a neighborhood of $e$ such that $X_{e}=x$, and $\mathcal{L}$ is the Lie derivation.

Example. Let $q \in \stackrel{k}{\otimes} \mathfrak{g}$. Set $Q=q^{\lambda}-q^{\rho}$, where

$$
q^{\lambda}(g)=g \cdot q, q^{\rho}(g)=q \cdot g,
$$

for $g \in G$. Then $Q$ is a multiplicative $k$-tensor. Such a multiplicative tensor is called exact because its associated 1-cocycle $\rho(Q): G \rightarrow \stackrel{k}{\otimes} \mathfrak{g}$ is exact, namely

$$
\rho(Q)(g)=A d_{g} q-q .
$$

(If an element $q \in \stackrel{k}{\otimes} \mathfrak{g}$ is considered as a 0 -cochain on $G$, its group coboundary is $\left.g \mapsto A d_{g} q-q.\right)$ In this case, $(D Q)(x)=a d_{x} q$, so that $D Q=\delta q$, the Lie-algebra coboundary of the 0 -cochain $q$, with respect to the adjoint representation.

\subsection{Poisson Lie groups and Lie bialgebras}

Definition. A Lie group $G$, with Poisson bivector $P$, is called a Poisson Lie group if $P$ is multiplicative.

By $(4.2), P_{e}=0$, so $(G, P)$ is not a symplectic manifold.

Examples. Obviously, $G$ with the trivial Poisson structure $(P=0)$ is a Poisson Lie group.

The dual $\mathfrak{g}^{*}$ of a Lie algebra, considered as an Abelian group, with the linear Poisson structure is a Poisson Lie group. In fact, in this case (4.1) reads

$$
P_{\xi+\eta}=P_{\xi}+P_{\eta},
$$

for $\xi, \eta \in \mathfrak{g}^{*}$, and this relation holds since $\xi \mapsto P_{\xi}$ is linear.

Remark. The condition that the Poisson bivector $P$ be multiplicative is equivalent to the following condition:

$$
\left\{\varphi \circ \lambda_{g}, \psi \circ \lambda_{g}\right\}(h)+\left\{\varphi \circ \rho_{h}, \psi \circ \rho_{h}\right\}(g)=\{\varphi, \psi\}(g h),
$$


for all functions $\varphi, \psi$ on $G$, and for all $g, h$ in $G$, which means that the multiplication map from $G \times G$ to $G$ maps Poisson brackets on $G \times G$ to Poisson brackets on $G$. In other words, the multiplication is a Poisson map from $G \times G$ to $G$, where $G \times G$ is endowed with the product Poisson structure. (We recall the definition of the product of Poisson manifolds and that of Poisson maps below, in Sect. 4.7.)

Let $r \in \bigwedge^{2} \mathfrak{g}$. Then the bivector defined by

$$
P=r^{\lambda}-r^{\rho}
$$

is multiplicative. Below we shall derive conditions on $r$ for $P$ to be a Poisson bivector.

When $P$ is a multiplicative bivector, let us set

$$
\gamma=D P=T_{e}(\rho(P))
$$

By the two propositions of Sect. 4.1, we know that $\gamma$ is a 1-cocycle of $\mathfrak{g}$ with values in $\bigwedge^{2} \mathfrak{g}$, with respect to the adjoint representation.

Proofs of the following two propositions will be given in Appendix 1.

Proposition. If $P$ is a multiplicative Poisson bivector on $G$, let $\gamma$ be defined by (4.10). Then ${ }^{t} \gamma: \bigwedge^{2} \mathfrak{g}^{*} \rightarrow \mathfrak{g}^{*}$ is a Lie bracket on $\mathfrak{g}^{*}$.

Then $(\mathfrak{g}, \gamma)$ is a Lie bialgebra, which is called the tangent Lie bialgebra of $(G, P)$.

Proposition. Conversely, if $(\mathfrak{g}, \gamma)$ is a Lie bialgebra, there exists a unique (up to isomorphism) connected and simply connected Poisson Lie group $(G, P)$ with tangent Lie bialgebra $(\mathfrak{g}, \gamma)$.

Here we shall be concerned only with the case where $P$ is defined by $r \in \bigwedge^{2} \mathfrak{g}$ by means of (4.9).

Proposition. The multiplicative bivector $P$ defined by (4.9) is a Poisson bivector if and only $\llbracket r, r \rrbracket$ is Ad-invariant.

Proof. Recall that $\llbracket r, r \rrbracket$ was defined in (2.4). The proof rests on the formulæ $\llbracket r^{\lambda}, r^{\lambda} \rrbracket=\llbracket r, r \rrbracket^{\lambda}, \quad \llbracket r^{\rho}, r^{\rho} \rrbracket=-\llbracket r, r \rrbracket^{\rho}$, and $\llbracket r^{\lambda}, r^{\rho} \rrbracket=0$, whence $\rho(\llbracket P, P \rrbracket)(g)=$ $A d_{g}(\llbracket r, r \rrbracket)-\llbracket r, r \rrbracket$.

If $G$ is connected, the $A d$-invariance under the action of $G$ is equivalent to the $a d$-invariance under the action of its Lie algebra $\mathfrak{g}$. (See Sect. 1.2 and 3.5 for the definitions of $a d$ - and $A d$-actions.) Thus, in this case, $P=r^{\lambda}-r^{\rho}$ is a Poisson bivector if and only if $r$ is a solution of the generalized Yang-Baxter equation. In particular, if $r$ is a triangular $r$-matrix, then $P$ is a Poisson bivector. (See Remark 1, below.) More generally, if $r$ is a quasi-triangular $r$-matrix with 
$a d$-invariant symmetric part $s$ and skew-symmetric part $a \in \bigwedge^{2} \mathfrak{g}$, such that $\langle a, a\rangle+\langle s, s\rangle=0$, then

$$
P=r^{\lambda}-r^{\rho}=a^{\lambda}-a^{\rho},
$$

and $P$ is a Poisson bivector since

$$
-\frac{1}{2} \llbracket a, a \rrbracket=\langle a, a\rangle=-\langle s, s\rangle,
$$

which is an ad-invariant element of $\bigwedge^{3} \mathfrak{g}$. Then $(G, P)$ is called a quasi-triangular Poisson Lie group. If moreover $\underline{s}$ is invertible, then the Poisson Lie group $(G, P)$ is called factorizable.

Thus any quasi-triangular $r$-matrix, in particular a triangular or a factorizable $r$-matrix, gives rise to a Poisson-Lie structure on $G$. The Poisson bracket of functions on $G$ thus defined is called the Sklyanin bracket or the quadratic bracket. The reason for this latter name will appear in the next section.

Remark 1. It is clear from the proof of the preceding proposition that, when $r$ is a triangular $r$-matrix, both $r^{\lambda}$ and $r^{\rho}$ are also Poisson structures. Moreover, they are compatible, i.e., any linear combination of $r^{\lambda}$ and $r^{\rho}$ is a Poisson structure. However, $r^{\lambda}-r^{\rho}$ is the only Poisson-Lie structure in this family.

Remark 2. When $r$ is quasi-triangular, $\langle a, a\rangle=-\langle s, s\rangle$ and therefore both $a^{\lambda}-a^{\rho}$ and $a^{\lambda}+a^{\rho}$ are Poisson structures. While $a^{\lambda}-a^{\rho}$ is a Poisson-Lie structure, $a^{\lambda}+a^{\rho}$ is not. In fact, its rank at the unit, $e$, is the rank of $\underline{a}$, and therefore not 0 , unless we are in the trivial case, $a=0$. If $\underline{a}$ is invertible, $a^{\lambda}+a^{\rho}$ is symplectic in a neighborhood of $e$.

\subsection{The second Russian formula (quadratic brackets)}

Let us assume that $G$ is a Lie group of $p \times p$ matrices. Then $\mathfrak{g}$ is a Lie algebra of $p \times p$ matrices and $r$ is a $p^{2} \times p^{2}$ matrix. If $L$ is a point in $G$, the entries of the left (resp., right) translate of $r$ by $L$ are those of the product of matrices $(L \otimes L) r($ resp., $r(L \otimes L))$. As in Sect. 3.3, we shall consider each entry $a_{i}^{j}$ of $L$ as the restriction to $G$ of the corresponding linear function on the space of $p \times p$ matrices, and we shall denote the table of their pairwise Poisson brackets, in the Poisson-Lie structure on $G$ defined by $r$, by $\{L, L\}$. The differential of such a linear function is constant and coincides with the linear function itself. Therefore the $\begin{array}{cc}j & \ell \\ i & k\end{array}$ coefficient of $\{L, L\}$ is the $\begin{array}{lll}j & \ell \\ i & k\end{array}$ coefficient of $(L \otimes L) r-r(L \otimes L)$. Whence, for $L \in G$,

$$
\{L \stackrel{\otimes}{, L}=[L \otimes L, r] .
$$

Formula (4.11) is what I call the second Russian formula. (It is close to, but different from (3.11) in Sect. 3.3.) It is clear from formula (4.11) that the 
Poisson brackets of any two entries of matrix $L$ are quadratic functions of the entries of $L$, which justifies the name "quadratic bracket". (Note that in formula (3.11) in Sect. 3.3, the Poisson bracket is linear.) Formula (4.11) is the basis of the $r$-matrix method for classical lattice integrable systems.

\subsection{Examples}

\subsubsection{Quasi-triangular structure of $S L(2, \mathbb{R})$.}

Let $G=S L(2, \mathbb{R})$ be the group of real $2 \times 2$ matrices with determinant 1 . We consider $r=\frac{1}{8}(H \otimes H+4 X \otimes Y)$, the factorizable $r$-matrix of Example 2 in Sect. 2.3, an element of $\mathfrak{s l}(2, \mathbb{R}) \otimes \mathfrak{s l}(2, \mathbb{R})$, with skew-symmetric part $r_{0}=\frac{1}{4}(X \otimes Y-Y \otimes X)$.

Let $L=\left(\begin{array}{ll}a & b \\ c & d\end{array}\right)$ be a generic element of $G$. Then $a d-b c=1$. By (4.11), we obtain

$$
\left(\begin{array}{llll}
\{a, a\} & \{a, b\} & \{b, a\} & \{b, b\} \\
\{a, c\} & \{a, d\} & \{b, c\} & \{b, d\} \\
\{c, a\} & \{c, b\} & \{d, a\} & \{d, b\} \\
\{c, c\} & \{c, d\} & \{d, c\} & \{d, d\}
\end{array}\right)=\left[L \otimes L, r_{0}\right]
$$

where

$$
L \otimes L=\left(\begin{array}{cccc}
a^{2} & a b & b a & b^{2} \\
a c & a d & b c & b d \\
c a & c b & d a & d b \\
c^{2} & c d & d c & d^{2}
\end{array}\right) \text { and } r_{0}=\frac{1}{4}\left(\begin{array}{cccc}
0 & 0 & 0 & 0 \\
0 & 0 & -1 & 0 \\
0 & 1 & 0 & 0 \\
0 & 0 & 0 & 0
\end{array}\right)
$$

Therefore we find the quadratic Poisson brackets,

$$
\begin{aligned}
& \{a, b\}=\frac{1}{4} a b,\{a, c\}=\frac{1}{4} a c,\{a, d\}=\frac{1}{2} b c, \\
& \{b, c\}=0, \quad\{b, d\}=\frac{1}{4} b d,\{c, d\}=\frac{1}{4} c d .
\end{aligned}
$$

Using the Leibniz rule for Poisson brackets, we find

$$
\{a, a d-b c\}=\{b, a d-b c\}=\{c, a d-b c\}=\{d, a d-b c\}=0 .
$$

Thus $a d-b c$ is a Casimir function for this Poisson structure, which is indeed defined on $S L(2, \mathbb{R})$. 


\subsubsection{Triangular structure of $S L(2, \mathbb{R})$.}

Another Poisson Lie strucutre on $S L(2, \mathbb{R})$ is defined by the triangular $r$-matrix, considered in Example 3 of Sect. 2.3,

$$
r=X \otimes H-H \otimes X=\left(\begin{array}{cccc}
0 & -1 & 1 & 0 \\
0 & 0 & 0 & -1 \\
0 & 0 & 0 & 1 \\
0 & 0 & 0 & 0
\end{array}\right) .
$$

Taking into account the constraint $a d-b c=1$, we find

$$
\begin{gathered}
\{a, b\}=1-a^{2},\{a, c\}=c^{2},\{a, d\}=c(-a+d) \\
\{b, c\}=c(a+d),\{b, d\}=d^{2}-1,\{c, d\}=-c^{2} .
\end{gathered}
$$

Here also we can show that $a d-b c$ is a Casimir function.

\subsubsection{Quasi-triangular structure of $S U(2)$.}

Let $G=S U(2)$, and $\mathfrak{g}=\mathfrak{s u}(2)$, with basis

$$
X=\frac{1}{2}\left(\begin{array}{cc}
0 & 1 \\
-1 & 0
\end{array}\right), Y=\frac{1}{2}\left(\begin{array}{cc}
0 & i \\
i & 0
\end{array}\right), Z=\frac{1}{2}\left(\begin{array}{cc}
i & 0 \\
0 & -i
\end{array}\right),
$$

and commutation relations

$$
[X, Y]=Z,[Y, Z]=X,[Z, X]=Y .
$$

Then $r=Y \otimes X-X \otimes Y$ is a skew-symmetric $r$-matrix. In fact

$$
\begin{gathered}
\langle r, r\rangle=\left[r_{12}, r_{13}\right]+\left[r_{12}, r_{23}\right]+\left[r_{13}, r_{23}\right] \\
=[X \otimes Y \otimes 1-Y \otimes X \otimes 1, X \otimes 1 \otimes Y-Y \otimes 1 \otimes X] \\
+[X \otimes Y \otimes 1-Y \otimes X \otimes 1,1 \otimes X \otimes Y-1 \otimes Y \otimes X] \\
+[X \otimes 1 \otimes Y-Y \otimes 1 \otimes X, 1 \otimes X \otimes Y-1 \otimes Y \otimes X] \\
=-Z \otimes Y \otimes X+Z \otimes X \otimes Y-X \otimes Z \otimes Y+Y \otimes Z \otimes X+X \otimes Y \otimes Z-Y \otimes X \otimes Z .
\end{gathered}
$$

Now,

$[X \otimes 1 \otimes 1,\langle r, r\rangle]=Y \otimes Y \otimes X-Y \otimes X \otimes Y+Z \otimes Z \otimes X-Z \otimes X \otimes Z$

$[1 \otimes X \otimes 1,\langle r, r\rangle]=-Z \otimes Z \otimes X+X \otimes X \otimes Y-X \otimes Y \otimes X+X \otimes Z \otimes Z$

$[1 \otimes 1 \otimes X,\langle r, r\rangle]=Z \otimes X \otimes Z-X \otimes Z \otimes Z-X \otimes Y \otimes Y+Y \otimes X \otimes Y$.

Thus $a d_{X}^{(3)}\langle r, r\rangle=0$, and, similarly, $a d_{Y}^{(3)}\langle r, r\rangle=0, a d_{Z}^{(3)}\langle r, r\rangle=0$. Thus $r$ satisfies the generalized Yang-Baxter equation. (This $r$-matrix is not triangular.) 
The Poisson brackets on $S U(2)$ defined by $r$, setting $L=\left(\begin{array}{cc}a & b \\ c & d\end{array}\right) \in$ $S U(2)$, are

$$
\begin{gathered}
\{a, b\}=i a b,\{a, c\}=i a c,\{a, d\}=2 i b c \\
\{b, c\}=0,\{b, d\}=i b d,\{c, d\}=i c d
\end{gathered}
$$

The Lie bracket defined by $r$ on $(\mathfrak{s u}(2))^{*}$, the dual of the Lie algebra $\mathfrak{s u}(2)$, is given by

$$
\left[Z^{*}, X^{*}\right]=X^{*},\left[Z^{*}, Y^{*}\right]=Y^{*},\left[X^{*}, Y^{*}\right]=0
$$

These examples can be generalized to yield Poisson-Lie structures on each simple Lie group (Drinfeld [16]) and on each compact Lie group (Lu and Weinstein [45]). Very interesting examples arise on loop groups (Drinfeld [16], Reyman and Semenov-Tian-Shansky [27]).

\subsection{The dual of a Poisson Lie group}

Since every Lie bialgebra has a dual Lie bialgebra and a double, we shall study the corresponding constructions at the Lie group level.

If $(G, P)$ is a Poisson Lie group, we consider its Lie bialgebra $\mathfrak{g}$ whose 1 cocycle is $\gamma=D P$ (Sect. 4.2). We denote the dual Lie bialgebra by $\left(\mathfrak{g}^{*}, \gamma\right)$. By the last proposition of Sect. 4.2, we know that there exists a unique connected and simply connected Poisson Lie group with Lie bialgebra $\left(\mathfrak{g}^{*}, \gamma\right)$. We denote it by $G^{*}$ and we call it the dual of $(G, P)$. More generally, any Poisson-Lie group with Lie bialgebra $\left(\mathfrak{g}^{*}, \gamma\right)$ is called a dual of $(G, P)$.

If $G$ itself is connected and simply connected, then the dual of $G^{*}$ is $G$ (since the dual of $\mathfrak{g}^{*}$ is $\mathfrak{g}$, because $\mathfrak{g}$ is finite-dimensional).

Example 1. If $P$ is the trivial Poisson structure on $G(P=0)$, then the Liealgebra structure of $\mathfrak{g}^{*}$ is Abelian and the dual group of $G$ is the Abelian group $\mathfrak{g}^{*}$ with its linear Poisson structure.

Example 2. Let $G=S L(2, \mathbb{R})$ with the Poisson structure defined by the quasitriangular $r$-matrix, with skew-symmetric part $\frac{1}{4}(X \otimes Y-Y \otimes X)$. We have seen in Sect. 1.7 that the dual $\mathfrak{g}^{*}$ of $\mathfrak{s l}(2, \mathbb{R})$ can be identified with

$$
\left\{(x, y) \in \mathfrak{b}_{-} \oplus \mathfrak{b}_{+} \mid \mathfrak{h}-\text { components of } x \text { and } y \text { are opposite }\right\}
$$

This result extends to $\mathfrak{s l}(n, \mathbb{R})$ equipped with the standard $r$-matrix. Thus, if $G=S L(n, \mathbb{R})$, then

$$
G^{*}=\left\{\left(L_{-}, L_{+}\right) \in B_{-} \times B_{+} \mid \text {diagonal elements of } L_{-} \text {and } L_{+} \text {are inverse }\right\} .
$$

Here $B_{+}$(resp., $B_{-}$) is the connected component of the group of upper (resp., lower) triangular matrices with determinant 1 . 
For example, for $n=2$,

$$
B_{+}=\left\{\left(\begin{array}{cc}
a & b \\
0 & \frac{1}{a}
\end{array}\right) \mid a>0, b \in \mathbb{R}\right\}
$$

and

$$
B_{-}=\left\{\left(\begin{array}{cc}
a & 0 \\
c & \frac{1}{a}
\end{array}\right) \mid a>0, c \in \mathbb{R}\right\}
$$

so that

$$
G^{*}=\left\{\left(\left(\begin{array}{cc}
a & b \\
0 & \frac{1}{a}
\end{array}\right),\left(\begin{array}{cc}
\frac{1}{a} & 0 \\
c & a
\end{array}\right)\right) \mid a>0, b, c \in \mathbb{R}\right\} .
$$

This 3-dimensional Lie group can be identified with

$$
S B(2, \mathbb{C})=\left\{\left(\begin{array}{cc}
\alpha & \beta+i \gamma \\
0 & \alpha^{-1}
\end{array}\right) \mid \alpha>0, \beta, \gamma \in \mathbb{R}\right\} .
$$

Example 3. The dual of the Lie bialgebra $\mathfrak{s u}(2)$ (see Sect. 1.7) can be integrated to a Poisson Lie group. The real Lie group $S B(2, \mathbb{C})$ defined above has the Lie algebra with basis $H=\left(\begin{array}{cc}1 & 0 \\ 0 & -1\end{array}\right), X=\left(\begin{array}{ll}0 & 1 \\ 0 & 0\end{array}\right), X^{\prime}=i X$, and commutation relations $[H, X]=2 X,\left[H, X^{\prime}\right]=2 X^{\prime},\left[X, X^{\prime}\right]=0$, and thus is isomorphic to $\mathfrak{s u}(2)^{*}$. Thus the dual of the Poisson Lie group $S U(2)$ is also the group $S B(2, \mathbb{C})$.

However, $(\mathfrak{s} r(2, \mathbb{R}))^{*}$ and $(\mathfrak{s u}(2))^{*}$ are not isomorphic as Lie bialgebras, so $(S L(2, \mathbb{R}))^{*}$ and $(S U(2))^{*}$, which are both isomorphic as Lie groups to $S B(2, \mathbb{C})$, do not have the same Poisson structure.

More generally (see Sect. 1.7), the dual of the compact form $\mathfrak{k}$ of a complex simple Lie algebra $\mathfrak{g}$ is a Lie algebra $\mathfrak{b}$ such that

$$
\mathfrak{g}^{\mathbb{R}}=\mathfrak{k} \oplus \mathfrak{b} .
$$

If $K$ is a compact Lie group, with Lie algebra $\mathfrak{k}$, then $K$ is a Poisson Lie group whose dual, $K^{*}$, is the connected and simply connected Lie group with Lie algebra $\mathfrak{b}$. Thus, in the Iwasawa decomposition, $G=K A N$, both $K$ and $A N$ are Poisson Lie groups in duality. (See Lu and Weinstein [45].)

\subsection{The double of a Poisson Lie group}

When $(G, P)$ is a Poisson Lie group, its tangent Lie bialgebra $(\mathfrak{g}, \gamma)$ has a double $\mathfrak{d}$, which is a factorizable Lie bialgebra. (See Sect. 2.5.)

The connected and simply connected Lie group $\mathcal{D}$ with Lie algebra $\mathfrak{d}$ is called the double of $(G, P)$. Since $\mathfrak{d}$ is a factorizable Lie bialgebra with $r$-matrix $r_{\mathfrak{d}}, \mathcal{D}$ is a factorizable Poisson Lie group, with Poisson structure $P_{\mathcal{D}}=r_{\mathfrak{d}}^{\lambda}-r_{\mathfrak{d}}^{\rho}$, where 
$\lambda$ and $\rho$ refer to left and right translations in the Lie group $\mathcal{D}$. More precisely, the double of $(G, P)$ is the Poisson Lie group $\left(\mathcal{D}, P_{\mathcal{D}}\right)$. Since $\mathfrak{g}$ and $\mathfrak{g}^{*}$ are Lie subalgebras of $\mathfrak{d}, G$ and $G^{*}$ are Lie subgroups of $\mathcal{D}$.

More generally, any Poisson Lie group with Lie algebra $\mathfrak{d}$ is called a double of $(G, P)$.

Example. When $G$ is a Lie group with trivial Poisson structure, we know that its dual is the Abelian Lie group $\mathfrak{g}^{*}$. A Lie group whose Lie algebra is the double of $\mathfrak{g}$, i.e., the semi-direct product of $\mathfrak{g}$ and $\mathfrak{g}^{*}$ with the coadjoint representation, is the Lie group $T^{*} G$, the cotangent bundle of $G$.

By Remark 2 of Sect. 4.2, $r_{\mathfrak{d}}^{\lambda}+r_{\mathfrak{d}}^{\rho}$ is also a Poisson structure on $\mathcal{D}$ (but not a Poisson-Lie structure). The Lie group $\mathcal{D}$ equipped with the Poisson structure $r_{\mathfrak{d}}^{\lambda}+r_{\mathfrak{d}}^{\rho}$ is called the Heisenberg double of $(G, P)$. This Poisson structure is actually symplectic in a neighborhood of the unit.

If the Poisson Lie group $(G, P)$ itself is defined by a factorizable $r$-matrix $r \in \mathfrak{g} \otimes \mathfrak{g}$, then we can describe its double in a simple way.

In fact, integrating the Lie-algebra morphisms described in Sect. 2.4 and 2.5 , we obtain morphisms of Lie groups. In particular, let $G_{R}$ be the connected and simply connected Lie group with Lie algebra $\mathfrak{g}_{R}$. Then the Lie-algebra morphisms $R_{+}$and $R_{-}$can be integrated to Lie-group morphisms $\mathcal{R}_{+}$and $\mathcal{R}_{-}$ from $G_{R}$ to $G$, and the pair $\mathcal{J}=\left(\mathcal{R}_{+}, \mathcal{R}_{-}\right)$defines an embedding of $G_{R}$ into the direct product $G \times G$. Locally, near the unit, the double of $G$ can be identified with the product of the manifolds $G$ and $G_{R}$, which are Lie subgroups of $G \times G$.

\subsection{Poisson actions}

Whereas a Hamiltonian action of a Lie group $G$ on a Poisson manifold $M$ is defined as a group action which preserves the Poisson structure, a Poisson action is an action of a Poisson Lie group on a Poisson manifold satisfying a different property expressed in terms of the Poisson bivectors of both the manifold and the group. When the Poisson structure of the group is trivial, i.e., vanishes, we recover the Hamiltonian actions.

Definition. Let $\left(G, P_{G}\right)$ be a Poisson Lie group and $\left(M, P_{M}\right)$ a Poisson manifold. An action $\alpha$ of $G$ on $M$ is called a Poisson action if $\alpha: G \times M \rightarrow M$, $(g, m) \mapsto g . m$, is a Poisson map.

Recall that, setting $\alpha(g, m)=g . m, \alpha$ is an action of $G$ on $M$ if, for $g, h \in$ $G, m \in M$,

$$
\begin{gathered}
g .(h . m)=(g h) . m, \\
e . m=m .
\end{gathered}
$$

Recall also that the Poisson bracket on $G \times M$ is defined by

$$
\{\Phi, \Psi\}_{G \times M}(g, m)=\{\Phi(., m), \Psi(., m)\}_{G}(g)+\{\Phi(g, .), \Psi(g, .)\}_{M}(m),
$$


for $\Phi, \Psi \in C^{\infty}(G \times M)$.

Finally, recall that a Poisson map $\alpha$ from a Poisson manifold $\left(N, P_{N}\right)$ to a Poisson manifold $\left(M, P_{M}\right)$ is a map such that

$$
\{\varphi \circ \alpha, \psi \circ \alpha\}_{N}=\{\varphi, \psi\}_{M} \circ \alpha \text {, for } \varphi, \psi \in C^{\infty}(M) .
$$

Thus $\alpha$ is a Poisson action if, for $g, h \in G, m \in M$,

$$
\{\varphi, \psi\}_{M}(g . m)=\left\{\varphi_{m}, \psi_{m}\right\}_{G}(g)+\left\{\varphi^{g}, \psi^{g}\right\}_{M}(m),
$$

where we have set $\varphi_{m}(g)=\varphi(g . m)$ and $\left(\varphi^{g}\right)(m)=\varphi(g . m)$. As expected, if $\{,\}_{G}=0,(4.12)$ reduces to the condition that, for each $g \in G$, the mapping $m \in M \mapsto g . m \in M$ be a Poisson map.

If $(G, P)$ is a Poisson Lie group, the left and right actions of $G$ on itself are Poisson actions. We shall give more examples of Poisson actions in Sect. 4.9.

We shall now give an infinitesimal criterion for Poisson actions. We know that an action $\alpha$ of $G$ on $M$ defines an action $\alpha^{\prime}$ of $\mathfrak{g}$ on $M, \alpha^{\prime}: x \in \mathfrak{g} \mapsto x_{M}$, where $x_{M}$ is the vector field on $M$ defined by

$$
x_{M}(m)=\left.\frac{d}{d t}(\exp (-t x) \cdot m)\right|_{t=0} .
$$

In fact, $\alpha^{\prime}$ maps $x \in \mathfrak{g}$ to a vector field $x_{M}$ on $M$ in such a way that

$$
[x, y]_{M}=\left[x_{M}, y_{M}\right], \quad \text { for } x, y \in \mathfrak{g} .
$$

Moreover we can extend $\alpha^{\prime}$ to a map from $\bigwedge^{2} \mathfrak{g}$ to the bivectors on $M$ by setting

$$
(x \wedge y)_{M}(m)=x_{M}(m) \wedge y_{M}(m),
$$

and, more generally still, we can extend $\alpha^{\prime}$ to a morphism of associative algebras from $\Lambda \mathfrak{g}$ to the algebra of fields of multivectors on $M$.

Let us still denote by $m$ the exterior powers of the differential at $g$ of the map $\alpha_{m}: g \mapsto g . m$ from $G$ to $M$, and by $g$. those of the map $\alpha^{g}: m \mapsto g . m$ from $M$ to $M$. With these notations, which we shall use in the proof of the following proposition, we can write

$$
\alpha^{\prime}(w)(m)=w_{M}(m)=(-1)^{|w|} w \cdot m,
$$

for any $w \in \bigwedge \mathfrak{g}$, where $|w|$ is the degree of $w$.

Proposition. Let $\left(G, P_{G}\right)$ be a connected Poisson Lie group, with associated 1-cocyle of $\mathfrak{g}$,

$$
\gamma=D P_{G}=T_{e}\left(\rho\left(P_{G}\right)\right): \mathfrak{g} \rightarrow \wedge^{2} \mathfrak{g},
$$

and let $\left(M, P_{M}\right)$ be a Poisson manifold. The action $\alpha: G \times M \rightarrow M$ is a Poisson action if and only if

$$
\mathcal{L}_{x_{M}}\left(P_{M}\right)=-(\gamma(x))_{M}
$$


for all $x \in \mathfrak{g}$, where $\mathcal{L}$ denotes the Lie derivation.

Proof. In fact, condition (4.12) is equivalent to

$$
\left(P_{M}\right)_{g \cdot m}=\left(P_{G}\right)_{g} \cdot m+g \cdot\left(P_{M}\right)_{m},
$$

where we have used the notations introduced above. We shall now show that (4.13) is the infinitesimal form of (4.14). From (4.14), we find

$$
\begin{gathered}
\mathcal{L}_{x_{M}}\left(P_{M}\right)(m)=\left.\frac{d}{d t}\left(\exp t x \cdot\left(P_{M}\right)_{\exp (-t x) \cdot m}\right)\right|_{t=0} \\
=\left.\frac{d}{d t}\left(\exp t x \cdot\left(\left(P_{G}\right)_{\exp (-t x)} \cdot m+\exp (-t x) \cdot\left(P_{M}\right)_{m}\right)\right)\right|_{t=0} \\
=\left.\frac{d}{d t}\left(\exp t x \cdot\left(\left(P_{G}\right)_{\exp (-t x)} \cdot m\right)\right)\right|_{t=0} .
\end{gathered}
$$

Because $\alpha$ is an action, $\alpha^{g} \circ \alpha_{m}=\alpha_{m} \circ \lambda_{g}$, where $\lambda_{g}$ is the left tanslation by $g$ in $G$. Thus,

$$
\exp t x \cdot\left(\left(P_{G}\right)_{\exp (-t x)} \cdot m\right)=\left(\exp t x \cdot\left(P_{G}\right)_{\exp (-t x)}\right) \cdot m .
$$

On the other hand, by definition,

$$
\gamma(x)=\mathcal{L}_{x^{\rho}}\left(P_{G}\right)(e)=\left.\frac{d}{d t}\left(\exp (-t x) \cdot\left(P_{G}\right)_{\exp t x}\right)\right|_{t=0},
$$

since the flow of a right-invariant vector field acts by left translations. Therefore, we obtain

$$
\mathcal{L}_{x_{M}}\left(P_{M}\right)(m)=-(\gamma(x)) \cdot m=-(\gamma(x))_{M}(m) .
$$

This computation also shows that, conversely, (4.13) implies (4.14) when $G$ is connected.

Definition. A Lie-algebra action $x \mapsto x_{M}$ is called an infinitesimal Poisson action of the Lie bialgebra $(\mathfrak{g}, \gamma)$ on $\left(M, P_{M}\right)$ if it satisfies (4.13).

\subsection{Momentum mapping}

Generalizing the momentum mapping for Hamiltonian actions, we adopt the following definition. Here $G^{*}$ is again the Poisson Lie group dual to $G$.

Definition. A map $J: M \rightarrow G^{*}$ is said to be a momentum mapping for the Poisson action $\alpha: G \times M \rightarrow M$ if, for all $x \in \mathfrak{g}$,

$$
x_{M}=\underline{P}_{M}\left(J^{*}\left(x^{\lambda}\right)\right),
$$

where $x^{\lambda}$ is the left-invariant differential 1-form on $G^{*}$ defined by the element $x \in \mathfrak{g}=\left(T_{e} G^{*}\right)^{*}$, and $J^{*}\left(x^{\lambda}\right)$ is the inverse image of $x^{\lambda}$ under $J$. 
If, in particular, $G$ is a Lie group with trivial Poisson structure, then $G^{*}=\mathfrak{g}^{*}$, the differential 1-form $x^{\lambda}$ is the constant 1-form $x$ on $\mathfrak{g}^{*}$, and

$$
J^{*}\left(x^{\lambda}\right)=d(J(x)), \text { where } J(x)(m)=\langle J(m), x\rangle .
$$

Thus, in this case, we recover the usual definition of a momentum mapping for a Hamiltonian action, $J: M \rightarrow \mathfrak{g}^{*}$, that is

$$
x_{M}=\underline{P}_{M}(d(J(x))),
$$

i.e., $x_{M}$ is the Hamiltonian vector field with Hamiltonian $J(x) \in C^{\infty}(M)$. Whence the name "non-Abelian Hamiltonian" also given to the momentum mapping in the case of a Poisson action.

\subsection{Dressing transformations}

We consider a Poisson Lie group $\left(G, P_{G}\right)$, its dual $\left(G^{*}, P_{G^{*}}\right)$ and its double $\mathcal{D}$. Their respective Lie algebras are $\mathfrak{g}, \mathfrak{g}^{*}$ and $\mathfrak{d}$.

For each $x \in \mathfrak{g}$, we consider the vector field on $G^{*}$,

$$
\ell(x)=\underline{P}_{G^{*}}\left(x^{\lambda}\right),
$$

where $x^{\lambda}$ is the left-invariant 1 -form on $G^{*}$ defined by $x \in \mathfrak{g}=\left(T_{e} G^{*}\right)^{*}$. Then

Theorem. (i) The map $x \mapsto \ell(x)=\underline{P}_{G^{*}}\left(x^{\lambda}\right)$ is an action of $\mathfrak{g}$ on $G^{*}$, whose linearization at $e$ is the coadjoint action of $\mathfrak{g}$ on $\mathfrak{g}^{*}$.

(ii) The action $x \mapsto \ell(x)$ is an infinitesimal Poisson action of the Lie bialgebra $\mathfrak{g}$ on the Poisson Lie group $G^{*}$.

A concise proof of this theorem will be given in Appendix 2.

This action is called the left infinitesimal dressing action of $\mathfrak{g}$ on $G^{*}$. In particular, when $G$ is a trivial Poisson Lie group, its dual group $G^{*}$ is the Abelian group $\mathfrak{g}^{*}$, and the left infinitesimal dressing action of $\mathfrak{g}$ on $\mathfrak{g}^{*}$ is given by the linear vector fields $\ell(x): \xi \in \mathfrak{g}^{*} \mapsto-a d_{x}^{*} \xi \in \mathfrak{g}^{*}$, for each $x \in \mathfrak{g}$. We can prove directly that $x \mapsto \ell(x)$ is a Lie-algebra morphism from $\mathfrak{g}$ to the Lie algebra of linear vector fields on $\mathfrak{g}^{*}$. In fact, applying $\ell([x, y])$ to the linear function $z \in\left(\mathfrak{g}^{*}\right)^{*}$, we find $\ell([x, y]) \cdot z=\ell(x) \cdot \ell(y) \cdot z-\ell(y) \cdot \ell(x) \cdot z$.

Similarly, the right infinitesimal dressing action of $\mathfrak{g}$ on $G^{*}$ is defined by

$$
x \mapsto r(x)=-\underline{P}_{G^{*}}\left(x^{\rho}\right),
$$

where $x^{\rho}$ is the right-invariant 1 -form on $G^{*}$ defined by $x \in \mathfrak{g}$, and its linearization is the opposite of the coadjoint action of $\mathfrak{g}$ on $\mathfrak{g}^{*}$.

The dressing vector fields $\ell(x)=\underline{P}_{G^{*}}\left(x^{\lambda}\right)$ have the following property, called twisted multiplicativity,

$$
\ell_{u v}(x)=u \cdot \ell_{v}(x)+\ell_{u}\left(A d_{v^{-1}}^{*} x\right) \cdot v,
$$


for $u, v \in G^{*}$, and an analogous property holds for the right dressing vector fields,

$$
r_{u v}(x)=r_{u}(x) \cdot v+u \cdot r_{v}\left(A d_{u}^{*} x\right) .
$$

Dually, we can define the left and right infinitesimal dressing actions of $\mathfrak{g}^{*}$ on $G$ by

$$
\xi \mapsto \underline{P}_{G}\left(\xi^{\lambda}\right) \text { and } \xi \mapsto-\underline{P}_{G}\left(\xi^{\rho}\right) .
$$

Integrating these infinitesimal Poisson actions, when these vector fields are complete, we obtain Poisson actions of $G$ on $G^{*}$, and of $G^{*}$ on $G$, called the left and right dressing actions.

In the rest of this section, we derive the main properties of the dressing transformations.

Proposition. The symplectic leaves of $G$ (resp., $\left.G^{*}\right)$ are the connected components of the orbits of the right or left dressing action of $G^{*}$ (resp., $G$ ).

Proof. This is clear from the definitions, since either the vector fields $\underline{P}_{G^{*}}\left(x^{\rho}\right)$ or the vector fields $\underline{P}_{G^{*}}\left(x^{\lambda}\right)$ span the tangent space to the symplectic leaves of $G^{*}$, and similarly for $G$.

Proposition. The momentum mapping for the left (resp., right) dressing action of $G$ on $G^{*}$ is the opposite of the identity map (resp., is the identity map) from $G^{*}$ to $G^{*}$.

The proof follows from the definitions, and there is a similar statement for the actions of $G^{*}$ on $G$.

If the dual $G^{*}$ is identified with a subset of the quotient of $\mathcal{D}$ under the right (resp., left) action of $G$, the left (resp., right) dressing action of $G$ on $G^{*}$ is identified with left- (resp., right-) multiplication by elements of $G$, and similarly for the actions of $G$ on $G^{*}$.

There is an alternate way of defining the dressing actions, which shows their relationship to the factorization problem encountered in Sect. 3.7.

Let $g$ be in $G$ and $u$ in $G^{*}$. We consider their product $u g$ in $\mathcal{D}$. Because $\mathfrak{d}=\mathfrak{g} \oplus \mathfrak{g}^{*}$, elements in $\mathcal{D}$ sufficiently near the unit can be decomposed in a unique way as a product of an element in $G$ and an element in $G^{*}$ (in this order). Applying this fact to $u g \in \mathcal{D}$, we see that there exist elements ${ }^{u} g \in G$ and $u^{g} \in G^{*}$ such that

$$
u g={ }^{u} g u^{g} .
$$

We thus define locally a left action of $G^{*}$ on $G$ and a right action of $G$ on $G^{*}$. In other words, the action of $u \in G^{*}$ on $g \in G$ (resp., the action of $g \in G$ on $\left.u \in G^{*}\right)$ is given by

$$
\left.(u, g) \mapsto(u g)_{G} \quad \text { (resp., }(u, g) \mapsto(u g)_{G^{*}}\right),
$$


where $(u g)_{G}$ (resp., $\left.(u g)_{G^{*}}\right)$ denotes the $G$-factor (resp., $G^{*}$-factor) of $u g \in \mathcal{D}$ as $g^{\prime} u^{\prime}$, with $g^{\prime} \in G, u^{\prime} \in G^{*}$.

In the same way, the product $g u \in \mathcal{D}$ can be uniquely decomposed (if it is sufficiently near the unit) into ${ }^{g} u g^{u}$, where ${ }^{g} u \in G^{*}$ and $g^{u} \in G$. So, by definition,

$$
g u={ }^{g} u g^{u} .
$$

In this way, we obtain (locally) a left action of $G$ on $G^{*}$ and a right action of $G^{*}$ on $G$.

Let us show that the left action of $G$ on $G^{*}$ is indeed a group action. By definition, for $g, h \in G, u \in G^{*}$,

$$
(g h) u={ }^{g h} u(g h)^{u}
$$

and

$$
g(h u)=g\left({ }^{h} u h^{u}\right)=\left(g^{h} u\right) h^{u}={ }^{g}\left({ }^{h} u\right) g^{\left({ }^{h} u\right)} h^{u} .
$$

These two equations imply

$$
{ }^{g h} u={ }^{g}\left({ }^{h} u\right),
$$

which is what was to be proved. They also imply the relation

$$
(g h)^{u}=g^{\left({ }^{h} u\right)} h^{u},
$$

which expresses the twisted multiplicativity property of the dressing transformations of the left action of $G$ on $G^{*}$. Analogous results hold for the three other actions which we have defined.

Proposition. The left and right actions of $G$ on $G^{*}$ and of $G^{*}$ on $G$ defined by (4.19) and (4.20) coincide with the dressing actions.

Proof. To prove that these actions coincide with the dressing actions defined above, it is enough to show that the associated infinitesimal actions coincide.

Recall that the Lie bracket on the double $\mathfrak{d}=\mathfrak{g} \oplus \mathfrak{g}^{*}$ satisfies

$$
[x, \xi]_{\mathfrak{o}}=-a d_{\xi}^{*} x+a d_{x}^{*} \xi,
$$

for $x \in \mathfrak{g}, \xi \in \mathfrak{g}^{*}$ (see Sect. 1.6). In the linearization of the left (resp., right) action of $G$ on $G^{*}$, the image of $(x, \xi) \in \mathfrak{g} \oplus \mathfrak{g}^{*}$ is the projection onto $\mathfrak{g}^{*}$ of $[x, \xi]_{\mathfrak{o}}$ (resp., $[\xi, x]_{\mathfrak{d}}$ ), i.e., the linearized action is the coadjoint action (resp., the opposite of the coadjoint action) of $\mathfrak{g}$ on $\mathfrak{g}^{*}$.

Similarily the linearized action of the left (resp., right) action of $G^{*}$ on $G$ is the coadjoint (resp., the opposite of the coadjoint action) of $\mathfrak{g}^{*}$ on $\mathfrak{g}$.

From relation (4.22) we deduce that the vector fields of the left infinitesimal action of $\mathfrak{g}$ on $G^{*}$ satisfy the twisted multiplicativity property (4.17). This fact 
and the fact that the linearized action is the coadjoint action of $\mathfrak{g}$ on $\mathfrak{g}^{*}$ permit identifying this infinitesimal action with the left infinitesimal dressing action of $\mathfrak{g}$ on $G^{*}$ (see Lu and Weinstein [45]).

The proofs for the right action of $G$ on $G^{*}$, and for the left and right actions of $G^{*}$ on $G$ are similar.

To conclude, we wish to relate the dressing transformations defined above with the formulæ expressing the dressing of $G$-valued fields satisfying a zerocurvature equation. (See Faddeev and Takhtajan [29], Babelon and Bernard [34].) The field equation expresses a compatibility condition for a linear system, the Lax representation, equivalent to a nonlinear soliton equation. This nonlinear equation admits a Hamiltonian formulation such that the Poisson brackets of the $\mathfrak{g}$-valued Lax matrix are expressed in terms of a factorizable $r$-matrix. The dressing transformations act on the $G$-valued fields and preserve the solutions of the field equation. This action is in fact a Poisson action of the dual group, $G^{*}$.

If the Poisson Lie structure of $G$ is defined by a factorizable $r$-matrix, the double, $\mathcal{D}$, is isomorphic to $G \times G$, with, as subgroups, the diagonal subgroup $\{(g, g) \mid g \in G\} \approx G$, and $\left\{\left(g_{+}, g_{-}\right) \mid g_{ \pm}=\mathcal{R}_{ \pm} h, h \in G\right\} \approx G^{*}$, with Lie algebras $\mathfrak{g}$ and $\left\{\left(\underline{r}_{+} x, \underline{r}_{-} x\right) \mid x \in \mathfrak{g}\right\} \approx \mathfrak{g}^{*}$, respectively (see Sect. 2.5 and 4.6).

In this case, the factorization problems consist in finding group elements $g^{\prime} \in G$ and $g_{ \pm}^{\prime}=\mathcal{R}_{ \pm} h^{\prime}, h^{\prime} \in G$, satisfying

$$
\left(g_{+}, g_{-}\right)(g, g)=\left(g^{\prime}, g^{\prime}\right)\left(g_{+}^{\prime}, g_{-}^{\prime}\right),
$$

or $g^{\prime} \in G$ and $g_{ \pm}^{\prime}=\mathcal{R}_{ \pm} h^{\prime}, h^{\prime} \in G$, satisfying

$$
(g, g)\left(g_{+}, g_{-}\right)=\left(g_{+}^{\prime}, g_{-}^{\prime}\right)\left(g^{\prime}, g^{\prime}\right) .
$$

Let us write the left action of $G^{*}$ on $G$ in this case. From $u g={ }^{u} g u^{g}$, we obtain from (4.23),

$$
g_{+} g=g^{\prime} g_{+}^{\prime}, \quad g_{-} g=g^{\prime} g_{-}^{\prime} .
$$

Eliminating $g^{\prime}$, we find that $g_{+}^{\prime}{ }^{-1} g_{-}^{\prime}$ is obtained from $g_{+}^{-1} g_{-}$by conjugation by $g^{-1}$, and that $g_{+}^{\prime}, g_{-}^{\prime}$ solve the factorization equation

$$
g_{+}^{\prime-1} g_{-}^{\prime}=g^{-1}\left(g_{+}{ }^{-1} g_{-}\right) g \text {. }
$$

It follows that the action of the element $\left(g_{+}, g_{-}\right) \in G^{*}$ on $g \in G$ is given by

$$
g^{\prime}=g_{+} g g_{+}^{\prime-1}=g_{-} g g_{-}^{\prime-1},
$$

where the group elements $g_{+}^{\prime}$ and $g_{-}^{\prime}$ solve the factorization equation (4.25).

Similarly for the right action of $G^{*}$ on $G$, we obtain the factorization equation

$$
g_{+}^{\prime} g_{-}^{\prime-1}=g\left(g_{+} g_{-}^{-1}\right) g^{-1}
$$


and the action of $\left(g_{+}, g_{-}\right) \in G^{*}$ on $g \in G$ is given by

$$
g^{\prime}=g_{+}^{\prime-1} g g_{+}=g_{-}^{\prime-1} g g_{-},
$$

where the group elements $g_{+}^{\prime}$ and $g_{-}^{\prime}$ solve the factorization equation (4.27).

Thus we recover the formula of the dressing transformation in Faddeev and Takhtajan [29] and in Babelon and Bernard [34]. (In the convention of [29], the $g_{-}^{\prime}$ considered here is replaced by its inverse, while in [34], the factorization equation is $g_{-}^{\prime-1} g_{+}^{\prime}=g\left(g_{-}{ }^{-1} g_{+}\right) g^{-1}$.)

\subsection{Bibliographical note}

Multiplicative fields of tensors, were introduced by Lu and Weinstein [45]. See Kosmann-Schwarzbach [39], Vaisman [30], Dazord and Sondaz [37]. The results of Sect. 4.2 are due to Drinfeld [15], and are further developed in KosmannSchwarzbach [38] [39], Verdier [31], and $\mathrm{Lu}$ and Weinstein [45]. For formula (4.11), see e.g., Takhtajan [29]. For the examples of Sect. 4.5, see [45] and Majid [46][24]. Poisson actions were introduced by Semenov-Tian-Shansky [19], who showed that they were needed to explain the properties of the dressing transformations in field theory. Their infinitesimal characterization is due to $\mathrm{Lu}$ and Weinstein [45]. The generalization of the momentum mapping to the case of Poisson actions is due to $\mathrm{Lu}$ [44], while Babelon and Bernard [34], who call it the "non-Abelian Hamiltonian", have shown that in the case of the dressing transformations of $G$-valued fields the momentum mapping is given by the monodromy matrix of the associated linear equation. For the properties of the dressing transformations, see Semenov-Tian-Shansky [19], Lu and Weinstein [45], Vaisman [30], Alekseev and Malkin [32] (also, Kosmann-Schwarzbach and Magri [40] for the infinitesimal dressing transformations). A comprehensive survey of results, including examples and further topics, such as affine Poisson Lie groups, is given by Reyman [26]. 
Appendix 1

\section{The 'Big Bracket' and its Applications}

Let $F$ be a finite-dimensional (complex or real) vector space, and let $F^{*}$ be its dual vector space. We consider the exterior algebra of the direct sum of $F$ and $F^{*}, \bigwedge\left(F \oplus F^{*}\right)=\bigoplus_{n=-2}^{\infty}\left(\underset{p+q=n}{\oplus}\left(\bigwedge^{q+1} F^{*} \otimes \bigwedge^{p+1} F\right)\right)$.

We say that an element of $\Lambda\left(F \oplus F^{*}\right)$ is of bidegree $(p, q)$ and of degree $n=p+q$ if it belongs to $\bigwedge^{q+1} F^{*} \oplus \bigwedge^{p+1} F$. Thus elements of the base field are of bidegree $(-1,-1)$, elements of $F$ (resp., $\left.F^{*}\right)$ are of bidegree $(0,-1)$ (resp., $(-1,0)$ ), and a linear map $\mu: \bigwedge^{2} F \rightarrow F$ (resp., $\left.\gamma: F \rightarrow \bigwedge^{2} F\right)$ can be considered to be an element of $\bigwedge^{2} F^{*} \otimes F$ (resp., $F^{*} \otimes \bigwedge^{2} F$ ) which is of bidegree $(0,1)$ (resp., $(1,0))$.

Proposition. On the graded vector space $\bigwedge\left(F \oplus F^{*}\right)$ there exists a unique graded Lie bracket, called the big bracket, such that

- if $x, y \in F,[x, y]=0$,

- if $\xi, \eta \in F^{*},[\xi, \eta]=0$,

- if $x \in F, \xi \in F^{*},[x, \xi]=\langle\xi, x\rangle$.

- if $u, v, w \in \bigwedge\left(F \oplus F^{*}\right)$ are of degrees $|u|,|v|$ and $|w|$, then

$$
[u, v \wedge w]=[u, v] \wedge w+(-1)^{|u||v|} v \wedge[u, w] .
$$

This last formula is called the graded Leibniz rule. The following proposition lists important properties of the big bracket.

Proposition. Let [, ] denote the big bracket. Then

(i) $\mu: \bigwedge^{2} F \rightarrow F$ is a Lie bracket if and only if $[\mu, \mu]=0$.

(ii) ${ }^{t} \gamma: \bigwedge^{2} F^{*} \rightarrow F^{*}$ is a Lie bracket if and only if $[\gamma, \gamma]=0$.

(iii) Let $\mathfrak{g}=(F, \mu)$ be a Lie algebra. Then $\gamma$ is a 1-cocycle of $\mathfrak{g}$ with values in $\bigwedge^{2} \mathfrak{g}$, where $\mathfrak{g}$ acts on $\bigwedge^{2} \mathfrak{g}$ by the adjoint action, if and only if $[\mu, \gamma]=0$.

The dual and the double of a Lie bialgebra. By the graded commutativity of the big bracket,

$$
[\gamma, \mu]=[\mu, \gamma]
$$

This equality proves the proposition of Sect. 1.5 without any computation. 
To prove the theorem of Sect. 1.6, we write, by the bilinearity and graded skew-symmetry of the big bracket,

$$
[\mu+\gamma, \mu+\gamma]=[\mu, \mu]+2[\mu, \gamma]+[\gamma, \gamma] .
$$

Using the bigrading of $\bigwedge\left(F \oplus F^{*}\right)$, we see that conditions

$$
[\mu+\gamma, \mu+\gamma]=0
$$

and

$$
[\mu, \mu]=0,[\mu, \gamma]=0,[\gamma, \gamma]=0
$$

are equivalent. The first is equivalent to the fact that $\mu+\gamma$ defines a Lie-algebra structure on $F \oplus F^{*}$ which leaves the canonical scalar product invariant and is such that $F$ and $F^{*}$ are Lie subalgebras, and the second is equivalent to the defining relations for the Lie bialgebra $(\mathfrak{g}, \gamma)$, where $\mathfrak{g}=(F, \mu)$. Therefore in the finite-dimensional case, Lie bialgebras are in 1-1 correspondence with Manin triples.

Q.E.D.

The following lemma is basic.

Lemma. Let $\mathfrak{g}=(F, \mu)$ be a Lie algebra. Then

a) $d_{\mu}: a \mapsto[\mu, a]$ is a derivation of degree 1 and of square 0 of the graded Lie algebra $\bigwedge\left(F \oplus F^{*}\right)$,

b) if $a \in \Lambda F$, then $d_{\mu} a=-\delta a$, where $\delta$ is the Lie algebra cohomology operator, c) for $a, b \in \bigwedge F$, let us set

$$
\llbracket a, b \rrbracket=[[a, \mu], b] .
$$

Then $\llbracket$, 』is a graded Lie bracket of degree 1 on $\wedge F$ extending the Lie bracket of $\mathfrak{g}$. If $a=b$ and $a \in \bigwedge^{2} F$, this bracket coincides with the quantity introduced in (2.4).

The bracket $\llbracket, \rrbracket$ is called the algebraic Schouten bracket of the exterior algebra of $\mathfrak{g}$.

Coboundary Lie bialgebras. We can now prove the second proposition in Sect. 2.1. Let $\gamma=\delta a=-d_{\mu} a=-[\mu, a]$, where $a \in \bigwedge^{2} \mathfrak{g}$. By the graded Jacobi identity,

$$
[\gamma, \gamma]=\left[d_{\mu} a, d_{\mu} a\right]=[[\mu, a],[\mu, a]]=[\mu,[a,[\mu, a]]]-[[\mu,[\mu, a]], a] .
$$

The second term vanishes because $[\mu, \mu]=0$, and therefore $[\mu,[\mu, a]]=0$. Now, by part c) of the lemma, we obtain

$$
[\gamma, \gamma]=d_{\mu} \llbracket a, a \rrbracket,
$$


and therefore, by (ii) above, $\gamma=\delta a$ is a Lie algebra structure on $\mathfrak{g}^{*}$ if and only if $\llbracket a, a \rrbracket$ is $a d$-invariant.

The tangent Lie bialgebra of a Poisson Lie group and the integration theorem. We now prove the propositions of Sect. 4.2. Let $(G, P)$ be a Poisson Lie group, and let $\gamma=D P=T_{e}(\rho(P))$. We can show that, because $P$ is multiplicative,

$$
[D P, D P]=-D \llbracket P, P \rrbracket .
$$

To prove this equality we decompose $P$ as a sum of decomposable bivectors and we use the biderivation property of the Schouten bracket.

It follows from this relation that, if $\llbracket P, P \rrbracket=0$, then $[\gamma, \gamma]=0$. Conversely, let $(\mathfrak{g}, \gamma)$ be a Lie bialgebra. Then $\gamma$ is a Lie algebra 1-cocycle and it can be integrated into a Lie group 1-cocycle, $\Gamma$, on the connected and simply connected Lie group $G$ with Lie algebra $\mathfrak{g}$. For $g \in G$, let $P_{g}=\Gamma(g) . g$. We thus define a multiplicative bivector, $P$, on $G$. Moreover, $P$ is a Poisson bivector. In fact, $\llbracket P, P \rrbracket$ is multiplicative (as the Schouten bracket of a multiplicative bivector) and, by the above relation, $[\gamma, \gamma]=0$ implies that $D \llbracket P, P \rrbracket=0$. This is enough (see Lu and Weinstein [45]) to prove that $\llbracket P, P \rrbracket=0$.

Manin pairs. If $\mathfrak{p}$ is a finite-dimensional Lie algebra with an invariant, nondegenerate scalar product, and if $\mathfrak{a}$ is an isotropic Lie subalgebra of $\mathfrak{p}$ of maximal dimension, then $(\mathfrak{p}, \mathfrak{a})$ is called a Manin pair. (The dimension of $\mathfrak{p}$ is necessarily even, and $\operatorname{dim} \mathfrak{a}=\frac{1}{2} \operatorname{dim} \mathfrak{p}$ ). If $\mathfrak{b}$ is only an isotropic subspace (not necessarily a Lie subalgebra) complementary to $\mathfrak{a}$, the corresponding structure on $\mathfrak{a}$ is called a Lie quasi-bialgebra or a Jacobian quasi-bialgebra. Lie quasi-bialgebras are generalizations of Lie bialgebras, which were defined by Drinfeld as the classical limit of quasi-Hopf algebras. The double of a Lie quasi-bialgebra is again a Lie quasi-bialgebra.

Twilled Lie algebras. A twilled Lie algebra (also called a double Lie algebra, but this is a definition different from that in Sect. 3.6, or a matched pair of Lie algebras) is just a Lie algebra that splits as the direct sum of two Lie subalgebras. In a twilled Lie algebra, each summand acts on the other by 'twisted derivations'. The double of a Lie bialgebra is a twilled Lie algebra in which the two summands are in duality, and the actions by twisted derivations are the coadjoint actions. There is a corresponding notion of a twilled Lie group (or double group or matched pair of Lie groups), in which each factor acts on the other. These actions have the property of twisted multiplicativity as in (4.22), and the vector fields of the associated infinitesimal action have a property of twisted multiplicativity as in (4.17). The double of a Poisson Lie group, $G$, is a twilled Lie group, with factors $G$ and $G^{*}$, and the dressing actions described in Sect. 4.9 are the action of one factor on the other. 
Bibliographical note. For the definition of the 'big bracket', see Kostant and Sternberg [10]. For its use in the theory of Lie bialgebras, see Lecomte et Roger [42], and see Kosmann-Schwarzbach [39] on which this Appendix is based. See [39] and Bangoura and Kosmann-Schwarzbach [36] for applications of the big bracket to the case of Lie quasi-bialgebras. For twilled Lie algebras and Lie groups, see Kosmann-Schwarzbach and Magri [40], Majid [46][24] and Lu and Weinstein [45].

\section{Appendix 2}

\section{The Poisson Calculus and its Applications}

We recall some basic facts from Poisson calculus, and we prove that the dressing vector fields define infinitesimal Poisson actions of $\mathfrak{g}$ on $G^{*}$ and of $\mathfrak{g}^{*}$ on $G$. The notations are those of Sections 3 and 4. If $(M, P)$ is a Poisson manifold, we denote the space of smooth functions on $M$ by $C^{\infty}(M)$. We further denote by $\{$,$\} the Poisson bracket defined by P$, and we set

$$
(\underline{P} \xi)(\eta)=P(\xi, \eta) .
$$

For any Poisson manifold $(M, P)$ there is a Lie bracket $[,]_{P}$ defined on the vector space of differential 1-forms,

$$
\llbracket \xi, \eta \rrbracket_{P}=\mathcal{L}_{\underline{P} \xi} \eta-\mathcal{L}_{\underline{P} \eta} \xi-d(P(\xi, \eta)) .
$$

This bracket is $\mathbb{R}$-linear, and it satisfies

$$
\llbracket \xi, f \eta \rrbracket_{P}=f \llbracket \xi, \eta \rrbracket_{P}+\left(\mathcal{L}_{\underline{P} \xi} f\right) \eta,
$$

for $f \in C^{\infty}(M)$. In fact, this Lie bracket is characterized by (A.2) together with the property,

$$
\llbracket d f, d g \rrbracket_{P}=d(\{f, g\})
$$

for $f, g \in C^{\infty}(M)$.

The linear mapping $\underline{P}$ from differential 1-forms to vector fields is a Liealgebra morphism, i.e., it satisfies

$$
\llbracket \underline{P} \xi, \underline{P} \eta \rrbracket=\underline{P}\left(\llbracket \xi, \eta \rrbracket_{P}\right) .
$$

Mapping $\underline{P}$ can be extended to a $C^{\infty}(M)$-linear mapping $\Lambda \underline{P}$ from differential forms of all degrees to fields of multivectors (skew-symmetric contravariant tensor fields), setting

$$
(\wedge \underline{P})\left(\xi_{1} \wedge \ldots \wedge \xi_{q}\right)=\underline{P} \xi_{1} \wedge \ldots \wedge \underline{P} \xi_{q} .
$$


The Schouten bracket $\llbracket, \rrbracket$ is a graded Lie bracket on the vector space of fields of multivectors, with its grading shifted by 1, extending the Lie bracket of vector fields and satisfying a graded version of the Leibniz rule, (A.8) below. More precisely, for $Q, Q^{\prime}, Q^{\prime \prime}$ multivectors of degrees $q, q^{\prime}, q^{\prime \prime}$, respectively,

$$
\llbracket Q, Q^{\prime} \rrbracket=-(-1)^{(q-1)\left(q^{\prime}-1\right)} \llbracket Q^{\prime}, Q \rrbracket,
$$

$$
\begin{aligned}
& \llbracket Q, \llbracket Q^{\prime}, Q^{\prime \prime} \rrbracket \rrbracket=\llbracket \llbracket Q, Q^{\prime} \rrbracket, Q^{\prime \prime} \rrbracket+(-1)^{(q-1)\left(q^{\prime}-1\right)} \llbracket Q^{\prime}, \llbracket Q, Q^{\prime \prime} \rrbracket \rrbracket, \\
& \llbracket Q, Q^{\prime} \wedge Q^{\prime \prime} \rrbracket=\llbracket Q, Q^{\prime} \rrbracket \wedge Q^{\prime \prime}+(-1)^{(q-1) q^{\prime}} Q^{\prime} \wedge \llbracket Q, Q^{\prime \prime} \rrbracket .
\end{aligned}
$$

The following properties are satisfied:

$$
\llbracket X, f \rrbracket=X \cdot f=\langle d f, X\rangle,
$$

for any vector field $X$, and

$$
\llbracket P, f \rrbracket=-\underline{P}(d f)
$$

When $P$ is a bivector, the bracket $\llbracket P, P \rrbracket$ coincides with the quantity introduced in (3.4).

Moreover, if $P$ is a Poisson bivector, the mapping $Q \mapsto \llbracket P, Q \rrbracket$ is a derivation of degree 1 and of square 0 of the associative, graded commutative algebra of multivectors, which we denote by $d_{P}$ and which we call the Lichnerowicz-Poisson differential.

Proposition. The linear map $\bigwedge(-\underline{P})$ intertwines the Lichnerowicz-Poisson differential and the de Rham differential of forms, $d$.

Proof. We have to show that, for any $q$-form $\xi$,

$$
d_{P}\left(\left(\wedge^{q} \underline{P}\right)(\xi)\right)=-\left(\wedge^{q+1} \underline{P}\right)(d \xi) .
$$

For $q=0$, this is just (A.10). When $q=1$ and $\xi=d f$, where $f \in C^{\infty}(M)$, then both sides vanish, since the Hamiltonian vector field $\underline{P}(d f)$ leaves $P$ invariant.

If $f \in C^{\infty}(M)$, then

$$
\begin{gathered}
d_{P}\left(\left(\wedge^{q} \underline{P}\right)(f \xi)\right)+\left(\wedge^{q+1} \underline{P}\right) d(f \xi) \\
=\llbracket P, f \rrbracket \wedge\left(\wedge^{q} \underline{P}\right)(\xi)+f \llbracket P,\left(\wedge^{q} \underline{P}\right)(\xi) \rrbracket+\left(\wedge^{q+1} \underline{P}\right)(d f \wedge \xi)+f\left(\wedge^{q+1} \underline{P}\right)(d \xi) \\
=f\left(\llbracket P,\left(\wedge^{q} \underline{P}\right)(\xi) \rrbracket+\left(\wedge^{q+1} \underline{P}\right)(d \xi)\right) .
\end{gathered}
$$

Therefore, (A.11) holds for all 1-forms. Since $d$ (resp., $d_{P}$ ) is a derivation of the associative, graded commutative algebra of differential forms (resp., multivectors), formula (A.11) holds for each integer $q \geq 0$. 
We also recall the following fact from Lie-group theory.

Lemma. Let $G$ be a Lie group with Lie algebra $\mathfrak{g}$. Then, for $\xi \in \mathfrak{g}^{*}, d\left(\xi^{\lambda}\right)=$ $(\delta \xi)^{\lambda}$ and $d\left(\xi^{\rho}\right)=-(\delta \xi)^{\rho}$, where $\delta \xi$ is the Lie algebra coboundary of the 1 cochain $\xi$ on $\mathfrak{g}$ with values in $\mathbb{R}$.

Explicitly,

$$
\delta \xi\left(x_{1}, x_{2}\right)=-\left\langle\xi,\left[x_{1}, x_{2}\right]\right\rangle
$$

so that

$$
\delta \xi=-{ }^{t} \mu(\xi),
$$

where $\mu: \bigwedge^{2} \mathfrak{g} \rightarrow \mathfrak{g}$ is the Lie bracket on $\mathfrak{g}$.

If $\mu$ is considered as an element in $\bigwedge^{2} \mathfrak{g}^{*} \otimes \mathfrak{g}$, then the linear mapping defined in terms of the big bracket (see Appendix 1), $d_{\mu}: \alpha \mapsto[\mu, \alpha]$ is a derivation of degree 1 and square 0 of the exterior algebra $\bigwedge\left(F \oplus F^{*}\right)$ and

$$
d_{\mu} \xi=[\mu, \xi]={ }^{t} \mu(\xi)=-\delta \xi .
$$

On a Poisson Lie group, the left-invariant 1-forms and the right-invariant 1-forms are Lie subalgebras of the space of differential 1-forms equipped with Lie bracket (A.1). More precisely

Proposition. Let $(G, P)$ be a Poisson Lie group with tangent Lie bialgebra $(\mathfrak{g}, \gamma)$ and set $[\xi, \eta]_{\mathfrak{g}^{*}}={ }^{t} \gamma(\xi \otimes \eta)$. Then, for all $\xi, \eta \in \mathfrak{g}^{*}$,

$$
\llbracket \xi^{\lambda}, \eta^{\lambda} \rrbracket_{P}=\left([\xi, \eta]_{\mathfrak{g}^{*}}\right)^{\lambda} \text { and } \llbracket \xi^{\rho}, \eta^{\rho} \rrbracket_{P}=\left([\xi, \eta]_{\mathfrak{g}^{*}}\right)^{\rho} \text {. }
$$

In other words, mappings $\xi \mapsto \xi^{\lambda}$ and $\xi \mapsto \xi^{\rho}$ are Lie-algebra morphisms from $\left(\mathfrak{g}^{*}, \gamma\right)$ to the Lie algebra of differential 1-forms on $(G, P)$.

Properties of the dressing actions. We are now able to give the proof of the theorem of Section 4.9 .

1. We prove that $\ell: x \mapsto \underline{P}_{G^{*}}\left(x^{\lambda}\right)$ is a Lie-algebra morphism from $\mathfrak{g}$ to the vector fields on $G^{*}$ with the usual Lie bracket of vector fields. In fact, by (A.12) applied to $\left(G^{*}, P_{G *}\right)$ and (A.4),

$$
\ell_{[x, y]}=\underline{P}_{G^{*}}\left([x, y]^{\lambda}\right)=\underline{P}_{G^{*}}\left(\llbracket x^{\lambda}, y^{\lambda} \rrbracket P_{G^{*}}\right)=\llbracket \underline{P}_{G^{*}}\left(x^{\lambda}\right), \underline{P}_{G^{*}}\left(y^{\lambda}\right) \rrbracket=\left[\ell_{x}, \ell_{y}\right] .
$$

Similarly, $r_{[x, y]}=-\left[r_{x}, r_{y}\right]$.

2. We show that the linearization of the dressing action of $G$ on $G^{*}$ is the coadjoint action of $\mathfrak{g}$ on $\mathfrak{g}^{*}$.

By definition, the linearization at a fixed point $m_{0}$ of the action $\alpha$ of Lie group $G$ on a manifold $M$ is the map $x \in \mathfrak{g} \mapsto \dot{\alpha}(x) \in \operatorname{End}\left(T_{m_{0}} M\right)$ which is the differential of the linearized action of $G$ on $T_{m_{0}} M$. Therefore,

$$
\dot{\alpha}(x)(v)=\left(\mathcal{L}_{x_{M}} V\right)\left(m_{0}\right)
$$


where $V$ is a vector field on $M$ with value $v$ at $m_{0}$. (Thus the endomorphism $\dot{\alpha}(x)$ of $T_{m_{0}} M$ associated with $x \in \mathfrak{g}$ is the linearization of the vector field $-x_{M}$, and the assignment $x \in \mathfrak{g} \mapsto \dot{\alpha}(x) \in \operatorname{End}\left(T_{m_{0}} M\right)$ is a morphism of Lie algebras, being the composition of two antimorphisms.)

Applying this fact to $M=G^{*}$, with $\alpha$ the dressing action of $G$ on $G^{*}$, and $x_{G^{*}}=\ell_{x}=\underline{P}_{G^{*}}\left(x^{\lambda}\right)$, we find, for $\xi \in T_{e} G^{*}=\mathfrak{g}^{*}$,

$$
x \cdot \xi=\mathcal{L}_{\ell_{x}}(\Xi)(e),
$$

where $\Xi$ is a vector field on $G^{*}$ with value $\xi$ at $e \in G^{*}$. We choose $\Xi=\xi^{\rho}$, and for $y \in \mathfrak{g}$, we compute,

$$
\begin{gathered}
\left\langle\mathcal{L}_{\ell_{x}} \xi^{\rho}, y^{\lambda}\right\rangle(e)=-\left\langle\mathcal{L}_{\xi^{\rho}}\left(\underline{P}_{G^{*}}\left(x^{\lambda}\right)\right), y^{\lambda}\right\rangle(e) \\
=-\mathcal{L}_{\xi^{\rho}}\left\langle\underline{P}_{G^{*}}\left(x^{\lambda}\right), y^{\lambda}\right\rangle(e)+\left\langle\underline{P}_{G^{*}}\left(x^{\lambda}\right), \mathcal{L}_{\xi^{\rho}}\left(y^{\lambda}\right)\right\rangle(e)=-\mathcal{L}_{\xi^{\rho}}\left(P_{G^{*}}\left(x^{\lambda}, y^{\lambda}\right)\right)(e) \\
=-\langle\xi, \mu(x, y)\rangle=\left\langle a d_{x}^{*} \xi, y\right\rangle .
\end{gathered}
$$

Therefore, $x \cdot \xi=a d_{x}^{*} \xi$.

A similar proof shows that the linearization of the right dressing action of $G$ on $G^{*}$ is $(x, \xi) \in \mathfrak{g} \times \mathfrak{g}^{*} \mapsto-a d_{x}^{*} \xi \in \mathfrak{g}^{*}$, and the proofs in the dual situation are identical.

3. To prove that $x \mapsto \ell_{x}=\underline{P}_{G^{*}}\left(x^{\lambda}\right)$ is an infinitesimal Poisson action we use (4.13). Thus we have to show that

$$
\mathcal{L}_{\ell_{x}}\left(P_{G^{*}}\right)=-(\gamma(x))_{G^{*}},
$$

where $\gamma$ is the linearization of $P_{G^{*}}$ at $e$ defining the Lie bracket of $\mathfrak{g}^{*}$, and

$$
(\gamma(x))_{G^{*}}=\wedge^{2} \underline{P}_{G^{*}}\left((\gamma(x))^{\lambda}\right) .
$$

Now by relation (A.11) and the Lemma,

$\mathcal{L}_{\ell_{x}}\left(P_{G^{*}}\right)=\llbracket \underline{P}_{G^{*}} x^{\lambda}, P_{G^{*}} \rrbracket=-d_{P_{G^{*}}}\left(\underline{P}_{G^{*}} x^{\lambda}\right)=\wedge^{2} \underline{P}_{G^{*}} d\left(x^{\lambda}\right)=-\wedge^{2} \underline{P}_{G^{*}}\left(\gamma(x)^{\lambda}\right)$.

The proofs for the right dressing action and in the dual case are similar. Thus, using the basic general properties of the Poisson calculus, we have obtained in the above formula a one-line proof of the Poisson property of the dressing actions.

Bibliographical note. For the Poisson calculus see Vaisman [30], or the earlier articles and book, Weinstein [50], K. H. Bhaskara and K. Viswanath, Calculus on Poisson Manifolds, Bull. London Math. Soc., 20, 68-72 (1988) and Poisson Algebras and Poisson Manifolds, Pitman Research Notes in Math., Longman 1988, and Kosmann-Schwarzbach and Magri [41]. (In [50], mapping $\pi$ is the 
opposite of $\underline{P}$ defined here, while the bracket $\{$,$\} coincides with \llbracket, \rrbracket_{P}$. In [41], mapping $\underline{P}$ and the bracket of 1 -forms are opposite to the ones defined in this Appendix). See also Lu and Weinstein [45].

\section{Selected Bibliography}

\section{Background on manifolds, Lie algebras and Lie groups, and Hamiltonian systems}

[1] R. Abraham, J.E. Marsden and T. Ratiu, Manifolds, Tensor Analysis and Applications, Springer-Verlag 1988.

[2] V. I. Arnold, Mathematical Methods of Classical Mechanics, 2nd ed., SpringerVerlag 1989 (in Russian, Nauka, Moscow 1974; French translation, Editions Mir, Moscou 1976).

[3] O. Barut and R. Raçzka, Theory of Group Representations and Applications, 2nd ed., World Scientific 1986.

[4] J. G. F. Belinfante and B. Kolman, A Survey of Lie Groups and Lie Algebras with Applications and Computational Methods, 3rd ed., SIAM Philadelphia 1992.

[5] Y. Choquet-Bruhat, C. DeWitt-Morette, M. Dillard-Bleick, Analysis, Manifolds and Physics, Part I (1982), Part II (1989), North-Holland.

[6] W. D. Curtis and F. R. Miller, Differential Manifolds and Theoretical Physics, Academic Press 1985.

[7] V. Guillemin and S. Sternberg, Symplectic Techniques in Physics, Cambridge University Press 1984.

[8] A. A. Kirillov, Elements of the Theory of Representations, Springer-Verlag 1975 (in Russian, Nauka, Moscow 1971; French translation, Editions Mir, Moscou 1974).

[9] B. Kostant, The solution to the generalized Toda lattice and representation theory, Adv. Math. 34, 195-338 (1979).

[10] B. Kostant and S. Sternberg, Symplectic reduction, BRS cohomology and infinite-dimensional Clifford algebras, Ann. Phys. (N.Y.) 176, 49-113 (1987).

[11] J.-L. Koszul, Homologie et cohomologie des algèbres de Lie, Bull. Soc. Math. France 78, 1-63 (1950).

[12] J. E. Marsden and T. S. Ratiu, Introduction to Mechanics and Symmetry, Springer-Verlag 1994; second edition 1999. 
[13] M. Postnikov, Lectures in Geometry, Semester V, Lie Goups and Lie Algebras, Mir, Moscow 1986 (in Russian, Nauka, Moscow 1982; French translation, Editions Mir, Moscou 1985).

[14] D. H. Sattinger and O. L. Sattinger, Lie Groups and Algebras with Applications to Physics, Geometry and Mechanics, Springer-Verlag 1986.

\section{A. Fundamental articles on Poisson Lie groups}

[15] V. G. Drinfeld, Hamiltonian Lie groups, Lie bialgebras and the geometric meaning of the classical Yang-Baxter equation, Sov. Math. Dokl. 27, ${ }^{\circ} 1,68-71$ (1983).

[16] V. G. Drinfeld, Quantum groups, in Proc. Intern. Cong. Math. Berkeley 1986, vol. 1, Amer. Math. Soc. (1987), pp. 798-820.

[17] I. M. Gelfand and I. Ya. Dorfman, Hamiltonian operators and the classical Yang-Baxter equation, Funct. Anal. Appl. 16, nº 4, 241-248 (1982).

[18] M. A. Semenov-Tian-Shansky, What is a classical r-matrix?, Funct. Anal. Appl. 17, n 4, 259-272 (1983).

[19] M. A. Semenov-Tian-Shansky, Dressing transformations and Poisson group actions, Publ. RIMS (Kyoto) 21, 1237-1260 (1985).

\section{B. Books and lectures on Poisson manifolds, Lie bialgebras, $r$-matrices and Poisson Lie groups}

[20] O. Babelon and C.-M. Viallet, Integrable Models, Yang-Baxter equation and quantum groups, SISSA Lecture Notes, 54 EP (1989).

[21] P. Cartier, Some fundamental techniques in the theory of integrable systems, in Lectures on Integrable Systems, In Memory of J.-L. Verdier, Proc. of the CIMPA School on Integrable Systems, Nice (France) 1991, O. Babelon, P. Cartier, Y. Kosmann-Schwarzbach, eds., World Scientific 1994, pp. 1-41. (Introduction to symplectic and Poisson geometry)

[22] V. Chari and A. Pressley, A Guide to Quantum Groups, Cambridge University Press 1994. (Chapters 1, 2, 3 and Appendix on simple Lie algebras)

[23] L. Faddeev and L. Takhtajan, Hamiltonian Methods in the Theory of Solitons, Springer-Verlag 1987.

[24] S. Majid, Foundations of Quantum Group Theory, Cambridge University Press 1995. (Chapter 8) 
[25] A. Perelomov, Integrable Systems of Classical Mechanics and Lie Algebras, Birkhäuser 1990.

[26] A. G. Reyman, Poisson structures related to quantum groups, in Quantum Groups and their Applications in Physics, Intern. School "Enrico Fermi" (Varenna 1994), L. Castellani and J. Wess, eds., IOS, Amsterdam 1996, pp. 407-443.

[27] A. Reyman and M. A. Semenov-Tian-Shansky, Group-theoretical methods in the theory of finite-dimensional integrable systems, in Dynamical Systems VII, V. I. Arnold and S. P. Novikov, eds., Springer-Verlag 1994 (Encycl. of Mathematical Sciences, vol. 16), pp. 116-225.

[28] M. A. Semenov-Tian-Shansky, Lectures on R-matrices, Poisson-Lie groups and integrable systems, in Lectures on Integrable Systems, In Memory of J.-L. Verdier, Proc. of the CIMPA School on Integrable Systems, Nice (France) 1991, O. Babelon, P. Cartier, Y. Kosmann-Schwarzbach, eds., World Scientific 1994, pp. 269-317. (Lectures on group-theoretical methods for integrable systems, Lie algebras, $r$-matrices, Poisson Lie groups)

[29] L. A. Takhtajan, Elementary course on quantum groups, in Lectures on Integrable Systems, In Memory of J.-L. Verdier, Proc. of the CIMPA School on Integrable Systems, Nice (France) 1991, O. Babelon, P. Cartier, Y. KosmannSchwarzbach, eds., World Scientific 1994, pp. 319-347. (Introduction to Poisson Lie groups and quantum groups)

[30] I. Vaisman, Lectures on the Geometry of Poisson Manifolds, Birkhäuser 1994.

[31] J.-L. Verdier, Groupes quantiques, d'après V. G. Drinfel'd, Séminaire Bourbaki, exposé 685, Astérisque 152-153, Soc. Math. Fr. 1987, pp. 305-319.

\section{Further developments on Lie bialgebras, $r$-matrices and Poisson Lie groups}

[32] A. Yu. Alekseev and A. Z. Malkin, Symplectic structures associated to Poisson-Lie groups, Comm. Math. Phys. 162, 147-173 (1994).

[33] R. Aminou, Y. Kosmann-Schwarzbach, Bigèbres de Lie, doubles et carrés, Ann. Inst. Henri Poincaré, Phys. Théor., 49A, nº4, 461-478 (1988).

[34] O. Babelon and D. Bernard, Dressing symmetries, Comm. Math. Phys. 149, 279-306 (1992).

[35] O. Babelon and C.-M. Viallet, Hamiltonian structures and Lax equations, Phys. Lett B 237, 411-416 (1990). 
[36] M. Bangoura and Y. Kosmann-Schwarzbach, The double of a Jacobian quasi-bialgebra, Lett. Math. Phys. 28, 13-29 (1993).

[37] P. Dazord and D. Sondaz, Groupes de Poisson affines, in Symplectic Geometry, Groupoids and Integrable Systems, P. Dazord and A.Weinstein, eds., Springer-Verlag 1991, pp. 99-128.

[38] Y. Kosmann-Schwarzbach, Poisson-Drinfeld groups, in Topics in Soliton Theory and Exactly Solvable Nonlinear Equations, M. Ablowitz, B. Fuchssteiner and M. Kruskal, eds., World Scientific 1987, pp. 191-215.

[39] Y. Kosmann-Schwarzbach, Jacobian quasi-bialgebras and quasi-Poisson Lie groups, Contemporary Mathematics 132, 459-489 (1992).

[40] Y. Kosmann-Schwarzbach and F. Magri, Poisson-Lie groups and complete integrability, I. Drinfeld bigebras, dual extensions and their canonical representations, Ann. Inst. Henri Poincaré, Phys. Théor., 49A, nº4, 433-460 (1988).

[41] Y. Kosmann-Schwarzbach and F. Magri, Poisson-Nijenhuis structures, Ann. Inst. Henri Poincaré, Phys. Théor., 53A, n 1, 35-81 (1990).

[42] P. Lecomte and C. Roger, Modules et cohomologie des bigèbres de Lie, Comptes rendus Acad. Sci. Paris 310, série I, 405-410 and 311, série I, 893-894 (1990).

[43] L. C. Li and S. Parmentier, Nonlinear Poisson structures and r-matrices, Comm. Math. Phys. 125, 545-563 (1989).

[44] J. H. Lu, Momentum mappings and reduction of Poisson actions, in Symplectic Geometry, Groupoids and Integrable Systems, P. Dazord and A.Weinstein, eds., Springer-Verlag 1991, pp. 209-226.

[45] J. H. Lu and A. Weinstein, Poisson Lie groups, dressing transformations and Bruhat decompositions, J. Diff. Geom. 31, 501-526 (1990).

[46] S. Majid, Matched pairs of Lie groups associated to solutions of the YangBaxter equations, Pacific J. Math. 141, 311-319 (1990).

[47] N. Yu. Reshetikhin and M. A. Semenov-Tian-Shansky, Quantum R-matrices and factorization problems, J. Geom. Phys. 5, 533-550 (1988).

[48] C. Roger, Algèbres de Lie graduées et quantification, in Symplectic Geometry and Mathematical Physics, P. Donato et al., eds., Birkhäuser 1991, pp. 374-421.

[49] Ya. Soibelman, On some problems in the theory of quantum groups, Advances in Soviet Mathematics 9, 3-55 (1992).

[50] A. Weinstein, Some remarks on dressing transformations, J. Fac. Sci. Univ. Tokyo IA, 35, nº1, 163-167 (1988). 


\section{Bibliographical note added in the second edition}

The theory of Lie bialgebras, $r$-matrices and Poisson Lie groups has continued to expand in the interval between the first and second editions of this book. Especially noteworthy is the expansion of the theory into the study of Lie bialgebroids and Poisson groupoids. More than fifty papers have appeared on Lie bialgebras and bialgebroids, while some thirty other papers deal primarily with Poisson Lie groups and Poisson groupoids. We mention only four useful references, where many more references can be found.

- Chapter I of the book Algebras of functions on quantum groups. Part I, by L. I. Korogodski and Y. S. Soibelman (Mathematical Surveys and Monographs, 56, American Mathematical Society, Providence, RI, 1998) covers much of the material presented here.

- The article Loop groups, R-matrices and separation of variables by J. Harnad, in Integrable Systems: from classical to quantum, CRM Proc. Lecture Notes, 26, Amer. Math. Soc., Providence, RI, 2000 p. 21-54, reviews the basics of the theory of classical $R$-matrices and presents important applications to the study of integrable systems.

- In the same volume, p. 165-188, Characteristic systems on Poisson Lie groups and their quantization, by N. Reshetikhin, includes a review of important results in the theory of Lie bialgebras, of Poisson Lie groups and their symplectic leaves, and presents applications to the theory of some dynamical systems generalizing the Toda system.

- The paper Symplectic leaves of complex reductive Poisson-Lie groups, by M. Yakimov, Duke Math. J. 112, 453-509 (2002), is an in-depth study of the geometry of some Poisson Lie groups using Lie theory .

The first and third references will also be useful for leads to the vast literature concering the quantization of Lie bialgebras. 